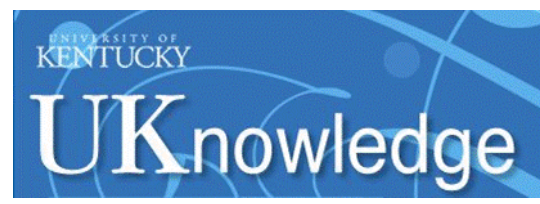

University of Kentucky

UKnowledge

\title{
Star Formation in Self-Gravitating Disks in Active Galactic Nuclei. II. Episodic Formation of Broad-Line Regions
}

\author{
Jian-Min Wang \\ Chinese Academy of Sciences, China \\ $\mathrm{Pu} \mathrm{Du}$ \\ Chinese Academy of Sciences, China \\ Jack A. Baldwin \\ Michigan State University \\ Jun-Qiang Ge \\ Chinese Academy of Sciences, China \\ Chen $\mathrm{Hu}$ \\ Chinese Academy of Sciences, China
}

See next page for additional authors

Follow this and additional works at: https://uknowledge.uky.edu/physastron_facpub

Part of the Astrophysics and Astronomy Commons, and the Physics Commons

Right click to open a feedback form in a new tab to let us know how this document benefits you.

\section{Repository Citation}

Wang, Jian-Min; Du, Pu; Baldwin, Jack A.; Ge, Jun-Qiang; Hu, Chen; and Ferland, Gary J., "Star Formation in Self-Gravitating Disks in Active Galactic Nuclei. II. Episodic Formation of Broad-Line Regions" (2012). Physics and Astronomy Faculty Publications. 68.

https://uknowledge.uky.edu/physastron_facpub/68

This Article is brought to you for free and open access by the Physics and Astronomy at UKnowledge. It has been accepted for inclusion in Physics and Astronomy Faculty Publications by an authorized administrator of UKnowledge. For more information, please contact UKnowledge@lsv.uky.edu. 
Star Formation in Self-Gravitating Disks in Active Galactic Nuclei. II. Episodic Formation of Broad-Line Regions

Digital Object Identifier (DOI)

https://doi.org/10.1088/0004-637X/746/2/137

Notes/Citation Information

Published in The Astrophysical Journal, v. 746, no. 2, 137, p. 1-27.

(C) 2012. The American Astronomical Society. All rights reserved. Printed in the U.S.A.

The copyright holder has granted permission for posting the article here.

Authors

Jian-Min Wang, Pu Du, Jack A. Baldwin, Jun-Qiang Ge, Chen Hu, and Gary J. Ferland 


\title{
STAR FORMATION IN SELF-GRAVITATING DISKS IN ACTIVE GALACTIC NUCLEI. II. EPISODIC FORMATION OF BROAD-LINE REGIONS
}

\author{
Jian-Min Wang ${ }^{1,2}$, Pu Du ${ }^{1}$, Jack A. Baldwin ${ }^{3}$, Jun-Qiang Ge ${ }^{1}$, Chen Hu$^{1}$, And Gary J. Ferland ${ }^{4}$ \\ ${ }^{1}$ Key Laboratory for Particle Astrophysics, Institute of High Energy Physics, Chinese Academy of Sciences, 19B Yuquan Road, Beijing 100049, China; \\ wangjm@mail.ihep.ac.cn \\ 2 National Astronomical Observatories of China, Chinese Academy of Sciences, 20A Datun Road, Beijing 100020, China \\ ${ }^{3}$ Physics and Astronomy Department, 3270 Biomedical Physical Sciences Building, Michigan State University, East Lansing, MI 48824, USA \\ ${ }^{4}$ Department of Physics and Astronomy, 177 Chemistry/Physics Building, University of Kentucky, Lexington, KY 40506, USA \\ Received 2011 September 29; accepted 2011 December 15; published 2012 February 1
}

\begin{abstract}
This is the second in a series of papers discussing the process and effects of star formation in the self-gravitating disk around the supermassive black holes in active galactic nuclei (AGNs). We have previously suggested that warm skins are formed above the star-forming (SF) disk through the diffusion of warm gas driven by supernova explosions. Here we study the evolution of the warm skins when they are exposed to the powerful radiation from the inner part of the accretion disk. The skins initially are heated to the Compton temperature, forming a Compton atmosphere (CAS) whose subsequent evolution is divided into four phases. Phase I is the duration of pure accumulation supplied by the SF disk. During phase II clouds begin to form due to line cooling and sink to the SF disk. Phase III is a period of preventing clouds from sinking to the SF disk through dynamic interaction between clouds and the CAS because of the CAS overdensity driven by continuous injection of warm gas from the SF disk. Finally, phase IV is an inevitable collapse of the entire CAS through line cooling. This CAS evolution drives the episodic appearance of broad-line regions (BLRs). We follow the formation of cold clouds through the thermal instability of the CAS during phases II and III, using linear analysis. Since the clouds are produced inside the CAS, the initial spatial distribution of newly formed clouds and angular momentum naturally follow the CAS dynamics, producing a flattened disk of clouds. The number of clouds in phases II and III can be estimated, as well as the filling factor of clouds in the BLR. Since the cooling function depends on the metallicity, the metallicity gradients that originate in the SF disk give rise to different properties of clouds in different radial regions. We find from the instability analysis that clouds have column density $N_{\mathrm{H}} \lesssim 10^{22} \mathrm{~cm}^{-2}$ in the metal-rich regions whereas they have $N_{\mathrm{H}} \gtrsim 10^{22} \mathrm{~cm}^{-2}$ in the metal-poor regions. The metal-rich clouds compose the high-ionization line regions whereas the metal-poor clouds are in low-ionization line (LIL) regions. Since metal-rich clouds are optically thin, they will be blown away by radiation pressure, forming the observed outflows. The outflowing clouds could set up a metallicity correlation between the BLRs and narrow-line regions. The LIL regions are episodic due to the mass cycle of clouds with the CAS in response to continuous injection by the SF disk, giving rise to different types of AGNs. Based on Sloan Digital Sky Survey quasar spectra, we identify a spectral sequence in light of emission-line equivalent width from phase I to IV. A key phase in the episodic appearance of the BLRs is bright type II AGNs with no or only weak BLRs, contrary to the popular picture in which the absence of a BLR is due to a low accretion rate. We discuss observational implications and tests of the theoretical predictions of this model.
\end{abstract}

Key words: black hole physics - galaxies: evolution - quasars: general

\section{INTRODUCTION}

Accretion onto supermassive black holes (SMBHs) liberates copious amounts of energy, mainly as big blue bumps and Xrays, driving many phenomena associated with active galactic nuclei (AGNs), such as broad emission lines and outflows. Numerous attempts have been made over the past four decades to construct a self-consistent model of AGNs, but these have not yet been successful and the structure of AGNs and quasars remains largely unsolved. Principle Component Analysis (PCA) for large samples of AGNs (Boroson \& Green 1992; vanden Berk et al. 2001; Marziani et al. 2003, 2010) has shown that the Eddington ratio is tightly correlated with eigenvector 1 and the properties of the broad emission lines. In addition, the broad-line region (BLR) metallicity relates with AGN luminosity or Eddington ratios, linking with the feeding process (Hamann \& Ferland 1999; Shemmer et al. 2004; Warner et al. 2004; Matsuoka et al. 2011). It is likely that accretion onto the SMBHs and the appearance of BLRs are accompanying processes resulting from supplying gas to the central engine, and the properties of the BLR are determined by the supplying processes.
Spectra of Seyfert galaxies and quasars generally show broad emission lines with full width at half-maximum (FWHM) of a few $10^{3} \mathrm{~km} \mathrm{~s}^{-1}$ (see the reviews by Osterbrock \& Mathews 1986; Netzer 1990; Sulentic et al. 2000; Ho 2008). BLRs are generally discussed as being composed of discrete rapidly moving clouds responsible for emitting the observed broad lines, although the smoothness of the observed profiles indicates that there must be large numbers of such clouds (Arav et al. 1997; Ferland 2004; Laor 2006; Laor et al. 2006).

It has been speculated for a long time that these clouds are formed from the thermal instability of the hot gas. However, some major questions remain open.

1. Why does the BLR form?

2. What are the origins of the emitting gas and its high metallicity?

3. What are properties of cold clouds formed by thermal instability?

4. What is the geometry of the BLR?

5. Do the BLR clouds relate to phenomenon of AGN outflows? 


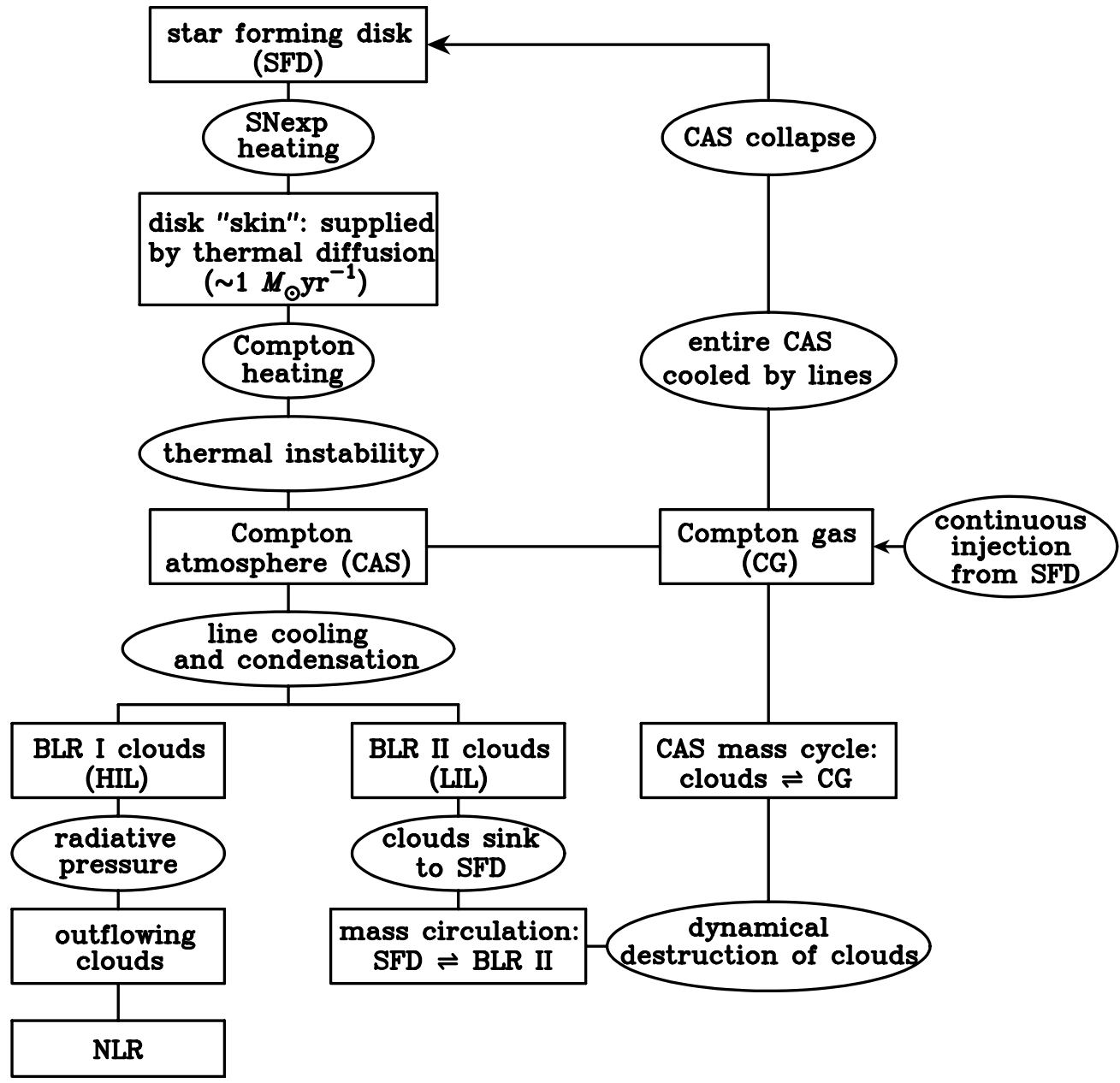

Figure 1. Flow chart of the model of episodic formation of broad-line regions presented in this paper. It starts with the SF disk, where evaporation is driving thermal diffusion of hot gas through SNexp. The diffuse gas then undergoes various physical processes, yielding broad-line regions as one of the by-products of feeding the SMBHs. The ellipses represent the underlying physics whereas the rectangles show states of the gas. The symbol " $\rightleftharpoons$ " indicates a cycle or a circulation of mass. See details in the main text of the paper.

The existing models listed below in Section 6 generally treat the above issues as being independent of each other, but there is increasing evidence that they are in fact closely related. First, the gravitational energy is mainly released around 3-100 Schwarzschild radii, but the well-known reverberation mapping relation of the strong correlation between BLR size and ionizing luminosity indicates $R_{\mathrm{BLR}} \propto L^{0.5}$ with $R_{\mathrm{BLR}} \sim 10^{4}$ Schwarzschild radii (Kaspi et al. 2000, 2005). The mapping relation is so strong that we believe there is an intrinsic connection between the accretion onto the SMBHs and BLR formation though the two scales are differing by a factor of $\sim 10^{2}$. The intrinsic connection could be complex somehow. If the broadening mechanism is due to an assembly of clouds with Keplerian rotation, the differences in width of different emission lines indicate that the BLR is an extended region. A flattened disk is then favored (Laor 2007; Collin et al. 2006). Second, it is well known that quasars are metal-rich, generally a few times the solar abundance and up to 10 or more times the solar value (Hamann \& Ferland 1992), and that higher metallicity correlates with higher luminosity, Eddington ratios, or SMBH mass (Hamann \& Ferland 1999; Shemmer et al. 2004; Netzer et al. 2004; Warner et al. 2004; Matsuoka et al. 2011). In particular, the metallicity does not relate with the IR luminosity (Simon \& Hamann 2010). All of these results provide potential clues for understanding the formation of BLRs and related phenomena, in the context of the BLR being a by-product of the feeding process. In addition, it has gradually been realized that star formation is not only important for feeding the SMBHs, but also for the metallicity observed in the BLRs (Wang et al. 2010, 2011).

The main goal of this paper is to make detailed predictions using a self-consistent model which addresses all of these aspects. Figure 1 sketches the global scenario of the model of BLR formation and evolution that we will develop and use here. For convenience, Table 1 lists the terminology used in this paper. Evaporation of molecular clouds driven by supernova explosions (SNexp) in the star-forming (SF) disk plays a key role in BLR formation. The gas evaporated by SNexp has a temperature of $10^{6} \mathrm{~K}$, but is still bound by the SMBH potential, forming a warm "skin" of the SF disk. Emission from the accretion disk around the SMBH heats the warm skin and establishes a "Compton atmosphere" (CAS) above the SF disk. The CAS is composed of cold clouds and Compton gas, whose fate is then determined by the interplay between Compton heating, cooling, and thermal instability, and by angular momentum redistribution, determining the BLR geometry and dynamics. The interaction between the clouds and the Compton gas, along with the continuous supply of warm gas from the SF disk, causes the clouds to have complicated lives, giving rise to an episodic appearance of the BLRs. The 
Table 1

Summary of Terminology Used in the Model

\begin{tabular}{ll}
\hline \hline Phrase & \multicolumn{1}{c}{ Physical Meanings } \\
\hline SMBH accretion disk & Accretion flows within the self-gravitating radius $\left(R_{\mathrm{SG}}\right)$ \\
$\begin{array}{l}\text { Star-forming disk (SFD) } \\
\text { Warm gas }\end{array}$ & Accretion flows between $R_{\mathrm{SG}}$ and the inner edge of dusty torus \\
Warm skin & Gas heated to $\sim 10^{6} \mathrm{~K}$ by the SNexp inside the SF disk \\
Compton atmosphere (CAS) & Warm gas bound by the SMBH potential \\
Compton gas (CG) & Is composed of Compton gas and cold clouds \\
Cold clouds & Hot gas at $T_{\text {Comp heated by AGN and with } \Xi_{1} \leqslant \Xi \leqslant \Xi_{2}(\text { see Section } 2.2 .4)}$ \\
Outflowing clouds & Clouds in the CAS that are formed through thermal instability \\
& Clouds driven by radiation pressure to move radially outward
\end{tabular}

advantages of the present model result from a natural assembly of a series of physical processes, providing a self-consistent solution that includes feeding the $\mathrm{SMBH}$, metal production, and BLR formation.

In a previous paper (Wang et al. 2011, hereafter W11), we showed that SNexp in the SF disk will expel gas that will then form a warm skin above the disk. This current paper continues on from that point and is structured as follows. Section 2 gives basic considerations for the underlying physical processes. Section 3 is devoted to discussing the fate of the atmosphere of the warm gas evaporated from the SF disk, and we find that there are four phases of the evolution driving the appearance of different types of AGNs. Section 4 discusses the high-ionization line (HIL) and low-ionization line (LIL) regions. Observational tests of the present model are extensively discussed in Section 5. In Section 6, we present a brief comparison of the current model with other existing models and point out future work that is needed. Finally, in Section 7, we summarize our conclusions.

\section{BASIC CONSIDERATIONS}

This section is devoted to basic considerations of the underlying physical processes of the atmosphere above the SF disk. After warm skins have inevitably developed above the SF disk as a result of the blast waves of SNexp (W11), they are exposed to the intensive radiation field of the accretion disk and are heated to produce ascending Compton gas. It should be emphasized that the skins are continuously supplied by the SF disk, resulting in a non-stationary state in the Compton gas. In addition, the rotating Compton gas will undergo thermal instability once its density is high enough, as well as having a dynamical interaction with the SMBHs. These competitive processes determine the basic properties of the BLR.

The SF disk is much larger than the $3-100 R_{\text {Sch }}$ region where most gravitational energy is released, where $R_{\mathrm{Sch}}=$ $2 G M_{\bullet} / c^{2}=3.0 \times 10^{13} M_{8} \mathrm{~cm}$ is the Schwarzschild radius, $G$ is the gravity constant, $M_{\bullet}=10^{8} M_{8} M_{\odot}$ is the SMBH mass, and $c$ is the light speed. For simplicity, we assume that the radiation field of the accretion disk is isotropic as a point energy source and that the SF disk has zero thickness.

\subsection{Gas Supply from the SF Disk: Warm Skins}

It is convenient to review some results from W11. Typical values of the parameters describing the SF disk are given in Table 1 of W11. The blast waves of SNexp interact with molecular clouds and evaporate warm gas (McKee \& Cowie 1975). The SF disk is assumed to be located in the region between the selfgravitating radius $\left(R_{\mathrm{SG}}\right)$ and the inner edge of the torus. The selfgravitating radius is given by $R_{\mathrm{SG}} \approx 511 \alpha^{2 / 9} M_{8}^{-2 / 9} \dot{m}^{4 / 9} R_{\mathrm{Sch}}$ (Laor \& Netzer 1989), where $\alpha$ is the viscosity parameter,
$\dot{m}=\dot{M} / \dot{M}_{\text {Edd }}, \dot{M}_{\text {Edd }}=2.22 \eta_{0.1}^{-1} M_{8} M_{\odot} \mathrm{yr}^{-1}$, and $\eta_{0.1}=\eta / 0.1$ is the radiative efficiency, assuming Toomre's parameter $Q=1$ (Goodman 2003; Rafikov 2009; Collin \& Zahn 2008). We take as the inner radius of the dusty torus $R_{\mathrm{torus}}=1.0 \mathrm{pc}$, for quasars with $M_{\bullet}=10^{8} M_{\odot}$. The density and temperature of the warm gas inside the SF disk are determined by the balance of heating and cooling. W11 give the density and temperature of the hot plasma in the SF disk as

$$
n_{6}=0.88 f_{\xi,-3}^{0.65} E_{51}^{0.65} \mu_{0}^{0.45} Z_{0}^{-0.36} \dot{\Sigma}_{*, 0}^{0.65} H_{14}^{-0.36},
$$

and

$$
T_{6}=1.40 f_{\xi,-3}^{0.23} \mu_{0}^{0.69} E_{51}^{0.23} Z_{0}^{0.23} \dot{\Sigma}_{*, 0}^{0.23} H_{14}^{0.23},
$$

where $n_{6}=n_{e} / 10^{6} \mathrm{~cm}^{-3}, T_{6}=T_{e} / 10^{6} \mathrm{~K}, Z_{0}=Z / Z_{\odot}$ is the metallicity, $E_{51}=E_{\mathrm{SN}} / 10^{51} \mathrm{erg}$ is the SNexp energy, $\dot{\Sigma}_{*, 0}$ is the surface density of star formation rate in units of $M_{\odot} \mathrm{yr}^{-1} \mathrm{pc}^{-2}$, $H_{14}=H_{\mathrm{d}} / 10^{14} \mathrm{~cm}$ is the thickness of the SF disk, and $f_{\xi}$ is a parameter related with the initial mass function in units of $10^{-3}$. The sound speed of the warm gas is $c_{s}=10^{7} T_{6}^{1 / 2} \mathrm{~cm} \mathrm{~s}^{-1}$, which is smaller than the escape velocity $V_{\mathrm{esc}}=4.2 \times 10^{8} r_{4}^{-1 / 2} \mathrm{~cm} \mathrm{~s}^{-1}$ (within $10^{4} R_{\mathrm{Sch}}$ ). Therefore, the warm gas is still bound by the SMBH potential, forming warm skins. The mass rate of warm gas injection through diffusion is then given by

$$
\begin{aligned}
\dot{M}_{\text {diff }}= & \int_{R_{\text {in }}}^{R_{\text {out }}} 2 \pi c_{s} m_{p} n_{e} R_{\mathrm{D}} d R_{\mathrm{D}} \\
= & 1.8 \int_{x_{\text {i. }}^{\prime}}^{x_{\text {out }}^{\prime}} f_{\xi,-3}^{0.77} E_{51}^{0.77} Z_{0}^{-0.24} \mu_{0}^{0.31} \dot{\Sigma}_{*, 0}^{0.77} \\
& \times H_{14}^{-0.24} x^{\prime} d x^{\prime} M_{\odot} \mathrm{yr}^{-1},
\end{aligned}
$$

where $R_{\mathrm{D}}$ is the radius of the SF disk, $x^{\prime}=R_{\mathrm{D}} / 10^{-0.5} \mathrm{pc}$, $R_{\text {in }}=R_{\mathrm{SG}}$, and $R_{\text {out }}=1 \mathrm{pc}$. We find that the total mass rates of the warm gas diffusion over the entire SF disk can be as high as a few $M_{\odot} \mathrm{yr}^{-1}$, which exceeds the Eddington limit for $10^{8} M_{\odot}$ SMBHs. It should be noted that the diffusion rates are much smaller than the inflow rate of the SF disk $\left(\sim 10 M_{\odot} \mathrm{yr}^{-1}\right.$; see Wang et al. 2010), and the skins have less significant influence on the SF disk itself.

The warm skins caused by the evaporation from the SF disk have the same angular momentum as the disk. The thickness of the warm skin is given by the vertical static equilibrium condition,

$$
H_{\text {skin }}=\frac{c_{s}}{\Omega_{\mathrm{K}}} \approx 1.29 \times 10^{16} T_{6}^{1 / 2} M_{8} r_{4}^{3 / 2} \mathrm{~cm},
$$

where $\Omega_{\mathrm{K}}=\left(G M_{\bullet} / R^{3}\right)^{1 / 2}$ is the Keplerian velocity, $r_{4}=$ $R / 10^{4} R_{\text {Sch }}$ and $T_{6}=T_{e} / 10^{6} \mathrm{~K}$. The relative height of the 
skin is $H_{\text {skin }} / R \approx 0.05 T_{6}^{1 / 2} r_{4}^{1 / 2}$, indicating that the skin is flattened. Since the skins are exposed to the central engine, they are undergoing AGN heating and expansion after dynamical adjustment.

The thermal diffusion of warm gas from the SF disk will continue as long as the pressure of warm gas in the disk is larger than the pressure of the CAS. The warm gas pressure is given by

$$
P_{\mathrm{SF}}=1.7 \times 10^{-4} f_{\xi,-3}^{0.88} \mu_{0}^{1.14} E_{51}^{0.88} Z_{0}^{-0.13} \dot{\Sigma}_{*, 0}^{0.88} H_{14}^{-0.13} \mathrm{dyncm}^{-2} .
$$

This equation indicates that $P_{\mathrm{SF}}$ is sensitive to the star formation rates, and insensitive to the metallicity and height of the disk. This pressure determines the mass of the BLR gas and its structure. We will show that the continuous injection driven by this pressure leads to a transient appearance of the BLR. In our discussion of the BLR formation, we assume a steady star formation rate in the SF disk during one episode of SMBH activity.

We would like to emphasize that the properties of the warm skin are governed by the star formation rate in the SF disk or by the surface density of the disk itself. Since the skins are totally influenced by the central engine, they just provide the boundary condition of the CAS for the BLR formation discussed later. The CAS undergoes a complicated evolution which drives the episodic appearance of a BLR even in response to continuous injection from the SF disk.

\subsection{Heating and Cooling Functions}

\subsubsection{Heating}

We mainly consider two processes of heating by the AGN continuum as the main mechanisms: Compton heating and photoionization. Assuming a point energy source with luminosity $L_{v}\left(=4 \pi R^{2} F_{v}\right)$ at the center, Compton heating rates are given by (Levich \& Sunyaev 1970)

$$
\begin{aligned}
\mathscr{H}_{\mathrm{Comp}}= & \frac{2 \pi \sigma_{\mathrm{T}} \hbar}{m_{e} c^{2}} \frac{1}{n_{e}} \int \nu F_{\nu} d \nu,=4.90 \times 10^{-28} n_{6}^{-1} r_{4}^{-2} M_{8}^{-2} \\
& \times \int_{10^{14} \mathrm{~Hz}}^{100 \mathrm{keV}}\left(\nu L_{v}\right)_{45} d \nu_{14} \mathrm{erg} \mathrm{cm}^{3} \mathrm{~s}^{-1}
\end{aligned}
$$

where $F_{v}$ is the photon flux of the AGN continuum (in $\left.\mathrm{erg} \mathrm{s}^{-1} \mathrm{~Hz}^{-1} \mathrm{~cm}^{-2}\right), \sigma_{\mathrm{T}}=6.65 \times 10^{-25} \mathrm{~cm}^{2}$ is the Thompson cross section, $\hbar=1.06 \times 10^{-27} \mathrm{erg} \mathrm{s}$ is the Planck constant, $m_{e}$ is the mass of the electron, and $n_{e}$ is the number density of electrons. Here, $\left(v L_{v}\right)_{45}=v L_{v} / 10^{45} \mathrm{erg} \mathrm{s}^{-1}, r_{4}=R / 10^{4} R_{\mathrm{Sch}}$, $R$ is the distance of the ionized gas to the center and $v_{14}=$ $v / 10^{14} \mathrm{~Hz}$. Here the Compton heating is simply expressed for a CAS with a constant density typical of that at the characteristic radius. We include the dependence on radius in following calculations. The heating rate due to photoionization is given by

$$
\mathscr{H}_{\mathrm{ph}} \approx 3.01 \times 10^{-23} T_{4}^{-0.4} \mathrm{erg} \mathrm{cm}^{3} \mathrm{~s}^{-1},
$$

where $T_{4}=T / 10^{4} \mathrm{~K}$ (Beltrametti 1981). We note that the photoionization heating rate must actually depend on the ionization parameter (defined by Equation (13)). Here we just use a simple approximation from the published literature that is roughly correct for the conditions in the BLR. Fortunately, Equation (7) does not affect the triggering thermal instability, although the final temperature of cold clouds depends on the ionization parameter. This simplified version of the heating will be improved in a future paper. The free-free absorption coefficient is $\tilde{\kappa}_{\mathrm{ff}}=1.32 \times 10^{-27} T_{4}^{-3 / 2} v_{10}^{-2} \mathrm{~cm}^{-4}$, where $v_{10}=v / 10^{10} \mathrm{~Hz}$ (Rybicki \& Lightman 1979), and only becomes significant at radio frequencies. Considering that the gravitational energy is originally released from optical to hard X-rays, the free-free absorption is neglected here though radio and IR photons can significantly heat the medium through this process (Ferland \& Baldwin 1999). However, for those low-luminosity AGNs (LLAGNs) powered by advection-dominated accretion flows (ADAFs), heating by radio emission cannot be neglected in the total energy budget of emission lines. In particular, clouds responsible for emission lines in low-ionization nuclear emissionline regions (LINERs) have been strongly influenced by the radio heating. However, we focus here on the radio-quiet quasars in which the radio emission is much fainter than the other bands. We have the total heating function as $\mathscr{H}=\mathscr{H}_{\mathrm{Comp}}+\mathscr{H}_{\mathrm{ph}}$.

\subsubsection{Cooling}

Three cooling processes are important: (1) Compton cooling, (2) free-free cooling, and (3) line cooling. The Compton cooling is given by

$$
\begin{aligned}
\mathscr{C}_{\text {Comp }}= & \frac{4 k T \sigma_{\mathrm{T}}}{m_{e} c^{2} n_{e}} \int F_{\nu} d \nu,=3.90 \times 10^{-27} r_{4}^{-2} M_{8}^{-2} n_{6}^{-1} T_{4} \\
& \times \int_{10^{14} \mathrm{~Hz}}^{100 \mathrm{keV}} L_{v, 31} d v_{14} \mathrm{erg} \mathrm{cm}^{3} \mathrm{~s}^{-1}
\end{aligned}
$$

where $L_{v, 31}=L_{v} / 10^{31} \mathrm{erg} \mathrm{s}^{-1} \mathrm{~Hz}^{-1}, v_{14}=v / 10^{14} \mathrm{~Hz}$, and the bremsstrahlung cooling is

$$
\mathscr{C}_{\mathrm{ff}}=2.4 \times 10^{-27} T^{1 / 2}=2.4 \times 10^{-25} T_{4}^{1 / 2} \mathrm{erg} \mathrm{cm}^{3} \mathrm{~s}^{-1} .
$$

The line cooling function has been extensively studied by many authors (e.g., Böhringer \& Hensler 1989; Sutherland \& Dopita 1993; Gnat \& Sternberg 2007). It is mainly characterized by three bumps, which are contributed by the elements $\mathrm{H}, \mathrm{He}(\mathrm{C}$, $\mathrm{N}, \mathrm{Si}, \mathrm{S}, \mathrm{Ne}, \mathrm{Mg}, \mathrm{O}$ ), and $\mathrm{H}$-like Fe ions, respectively. We fit each of these peaks by parabolic curves as approximations of the line cooling functions shown in Figure 2 in Böhringer \& Hensler (1989). The line cooling function for a plasma with the abundance $Z$ is

$$
\mathscr{C}_{\text {line }}=\sum_{i} 10^{f_{i}}+\left(\frac{Z}{Z_{\odot}}\right) \sum_{j} 10^{f_{j}} \operatorname{erg~cm}^{3} \mathrm{~s}^{-1}
$$

where $i=\mathrm{H}, \mathrm{He}, j=\mathrm{C}, \mathrm{O}, \mathrm{Ne}, \mathrm{Fe}, \mathrm{Fe}^{25+}$. We set a form of the cooling function as $f_{k}=a_{1}+a_{2} \exp \left[-\left(\log T-b_{1}\right)^{2} / b_{2}\right]+$ $a_{3} \exp \left[-\left(\log T-c_{1}\right)^{2} / c_{2}\right]$, where $k=i, j$, to fit the numerical cooling functions given by Böhringer \& Hensler (1989). The coefficients for different elements are listed by a sequence of $\left(a_{1}, a_{2}, a_{3}, b_{1}, b_{2}, c_{1}, c_{2}\right)$. We have $\mathrm{H}:(-59.37,36.21,6.47$, 4.97, 4.93, 4.01, 0.26); He: $(-30.34,0.81,7.65,4.90,0.056$, $5.11,3.03)$; C: $(-24.64,2.59,0.79,4.77,0.42,5.03,0.052)$; O: $(-23.00,1.39,0.96,5.11,0.15,5.44,0.043)$; Ne: $(-23.58$, 1.13, 1.29, 5.43, 0.051, 5.73, 0.044); Fe: (-25.21, 1.26, 2.59, $0.013,81.49,5.99,0.35) ; \mathrm{Fe}^{25+}:(-23.79,0.51,-0.032,7.03$, $0.047,3.34,15.81)$ for a plasma with solar abundances. This approximation is accurate to within $10 \%$ over the whole domain of temperatures from $10^{4}$ to $10^{7} \mathrm{~K}$, but it does not apply free-free emission of fully ionized gas $\left(>10^{8} \mathrm{~K}\right)$. This is sufficient for the purposes of the present paper. The total cooling functions are then $\mathscr{C}=\mathscr{C}_{\text {comp }}+\mathscr{C}_{\text {ff }}+\mathscr{C}_{\text {line }}$. 
We wish to stress the important role of metallicity in the cooling. The metallicity gradient suggested by W11 implies different cooling functions at different radii. As shown by subsequent Figure 3, the inner regions are overheated, if they are metal-poor, so that cold clouds are forbidden to form. A proper metallicity is necessary at a radius to cool the heating so that formation of clouds is permitted at this distance. The gradients give rise to different properties of cold clouds as a function of distance from the black hole.

\subsubsection{Compton Temperature}

The SEDs of radio-quiet quasars and AGNs are characterized by two humps: ${ }^{5}$ (1) the so-called big blue bump extending from optical to soft X-rays and (2) hard X-rays with a cutoff at about $100 \mathrm{keV}$ (e.g., Richards et al. 2006; Vasudevan et al. 2009; Grupe et al. 2010). The Compton temperature is determined by the photon mean energy $(\langle\epsilon\rangle)$ of the SED in the $1.0 \mathrm{pc}$ region. The hard X-ray SED is given by $F_{\mathrm{HX}}(E) \propto E^{-\alpha_{\mathrm{HX}}}$, where $\alpha_{\mathrm{HX}}=1.2-1.7$ with a cutoff at roughly $100-200 \mathrm{keV}$ (with the exception of the narrow-line Seyfert 1 galaxies in Grupe et al.'s (2010) sample, which have quite soft spectra in hard X-rays). The optical-UV SED has index $\alpha_{\mathrm{OUV}}=1.5$ and a cutoff at $0.1 \mathrm{keV}$. For this mean quasar SED, the Compton temperature is

$$
T_{\text {Comp }}=\frac{1}{4 k}\langle\epsilon\rangle=\mathrm{a} \text { few } \times 10^{7} \mathrm{~K},
$$

agreeing with Netzer (2008), where $k$ is the Boltzmann constant, in light of the SED. This Compton temperature is at or slightly above the critical values below which serious absorption appears in soft X-rays (e.g., Petre et al. 1984; Mathews \& Ferland 1987). We use the Compton temperature $T_{\text {Comp }}=3 \times 10^{7} \mathrm{~K}$ in this paper, which is generally lower than the virial temperature given by $T_{\mathrm{vir}}=3.63 \times 10^{12} r^{-1} \mathrm{~K}$, indicating the diffuse gas is still bound by the potential of the SMBHs. This heated gas forms an atmosphere above the SF disk.

The timescale of Compton heating is

$$
t_{\mathrm{Comp}}=\frac{3 m_{e} c^{2}}{4 \sigma_{\mathrm{T}}} \frac{4 \pi R^{2}}{\ell_{\mathrm{Edd}} L_{\mathrm{Edd}}}=\left\{\begin{array}{l}
8.54 \times 10^{2} r_{5}^{2} \ell_{0.3}^{-1} M_{8} \mathrm{yr} \\
8.54 r_{4}^{2} \ell_{0.3}^{-1} M_{8} \mathrm{yr} \\
0.085 r_{3}^{2} \ell_{0.3}^{-1} M_{8} \mathrm{yr}
\end{array}\right.
$$

where $r_{3}=R / 10^{3} R_{\mathrm{Sch}}, r_{5}=R / 10^{5} R_{\mathrm{Sch}}, R$ is the distance from the SMBH, $\ell_{0.3}=\ell_{\mathrm{Edd}} / 0.3$ and $\ell_{\mathrm{Edd}}=L_{\mathrm{Bol}} / L_{\mathrm{Edd}}$ is the Eddington ratio, and $L_{\mathrm{Bol}}$ is the bolometric luminosity. This timescale is very important for determining the fate of the warm skins above the SF disk.

From Figure 3, we find that the Compton temperature of the CAS slightly increases with metallicity, that is to say, it is a function of the radial distance from the black hole. From the figure, we find the approximate dependence $T_{\text {Comp }} \propto R^{-0.3}$. The CAS is not an exactly isothermal atmosphere. It should be pointed out that formation of clouds is a local event in the CAS, and the global properties of the CAS hardly affect cloud formation.

\footnotetext{
5 The present $\langle\epsilon\rangle$ excludes the additional humps at infrared and radio wavelengths since they do not originate in the central $1.0 \mathrm{pc}$ region of interest here. Photons from the SF disk are also neglected when determining the Compton temperature since they are dominated by photons from the accretion disk.
}

\subsubsection{Ionization Parameter}

The ionization parameter of the gas supplied by the SF disk is

$$
\Xi=\frac{L_{\text {ion }}}{4 \pi R^{2} c n_{e} k T}=2.2 r_{4}^{-2} L_{45}\left(n_{e} T_{e}\right)_{14}^{-1} M_{8}^{-2},
$$

where $L_{45}=L_{\mathrm{ion}} / 10^{45} \mathrm{erg} \mathrm{s}^{-1}$ is the ionizing luminosity and $\left(n_{e} T_{e}\right)_{14}$ is in units of $10^{14} \mathrm{~cm}^{-3} \mathrm{~K}$. This definition is very convenient for discussing the two-phase model with a pressure balance. It is well known that the S-shaped relation between $\Xi$ and $T$ shows the thermal states of the ionized plasma (Krolik et al. 1981). When $\Xi \gtrsim 10$, the ionized plasma will have only one hot phase with Compton temperature. Only a cold phase exists when $\Xi \lesssim 0.1$.

Figure 2(b) shows a comparison of the Compton and virial temperatures, indicating that the Compton gas is not able to escape from the SMBH potential. In the presence of continuous injection due to thermal diffusion, the Compton gas accumulates and its density increases. The ionization parameter of the Compton gas will drop until thermal instability develops. As we argue below, a two-phase medium will form in the Compton gas once the gas injected from the SF disk is sufficiently dense.

The ionization parameter is a strong function of the distance to the black hole. As a convenient estimate of the thermal state of the ionized gas, we divide the regions into two parts with the boundary at $\Xi=1$, corresponding to $R=10^{4} R_{\mathrm{Sch}}$. The inner part, known as the HIL region, has higher values of the ionization parameter with typical $\log \Xi=0.5$. The outer part, called the LIL region, is characterized here by the lower value $\log \Xi=-0.5$. Figure 4 illustrates the HIL and LIL regions. Detailed discussions are given in Sections 3.4.3, 4.2, and 4.3. Another definition of ionization parameter as $U=\left(4 \pi R^{2} c n_{e}\right)^{-1} \int L_{\nu} d v / h v$ (Osterbrock \& Ferland 2006) is often used to distinguish the LIL and HIL regions, with $U>10^{-2}$ corresponding to the HIL region (e.g., Marziani et al. 2010).

\subsubsection{Thermal Conduction}

Thermal conduction in the CAS plays a key role in governing the growth of instability. Its importance can be assessed by considering the heating rate for conduction $\mathcal{H}_{\text {con }}=$ $\nabla \cdot\left(\kappa_{\mathrm{S}} T^{2.5} \nabla T\right) \sim \kappa_{\mathrm{S}} T^{3.5} / \delta R^{2}$, where $\delta R$ is the scale of the perturbation length of the temperature, and $\kappa_{\mathrm{S}}=5.4 \times$ $10^{-7} \mathrm{erg} \mathrm{s}^{-1} \mathrm{~cm}^{-1} \mathrm{~K}^{-7 / 2}$ is Spitzer's coefficient of thermal conductivity (Spitzer 1962). Comparing it with the free-free cooling rate gives $\mathcal{H}_{\text {con }} / n^{2} \mathscr{C}_{\text {ff }} \sim 0.23 T_{7}^{3} \delta R_{14}^{-2} n_{7}^{-2}$, where $\delta R_{14}=\delta R / 10^{14} \mathrm{~cm}$ is the initial length of the thermal perturbation, while its ratio to the photoionization heating rate is $\mathcal{H}_{\text {con }} / n^{2} \mathscr{H}_{\mathrm{ph}} \sim 1.0 T_{7}^{3.9} \delta R_{14}^{-2} n_{7}^{-2}$, showing that conduction must be considered in the energy balance. When the perturbation length $\delta R$ is smaller than $10^{14} \mathrm{~cm}$, thermal conduction will smear the perturbation within the thermal timescale and prevent development of the thermal instability and formation of clouds.

As a summary, we have the net heating function

$$
\mathscr{L}=\mathscr{H}_{\text {Comp }}+\mathscr{H}_{\mathrm{ph}}-\mathscr{C}_{\text {Comp }}-\mathscr{C}_{\mathrm{ff}}-\mathscr{C}_{\text {line }},
$$

which are approximated by Equations (6)-(10) for a hot plasma with temperature of $T>10^{4} \mathrm{~K}$. It should be noted that unlike other processes, thermal conduction is excluded in this equation, but it appears in Equation (33) since it only happens between a hot and a cold medium. Thermal instability of isobaric gas at a 

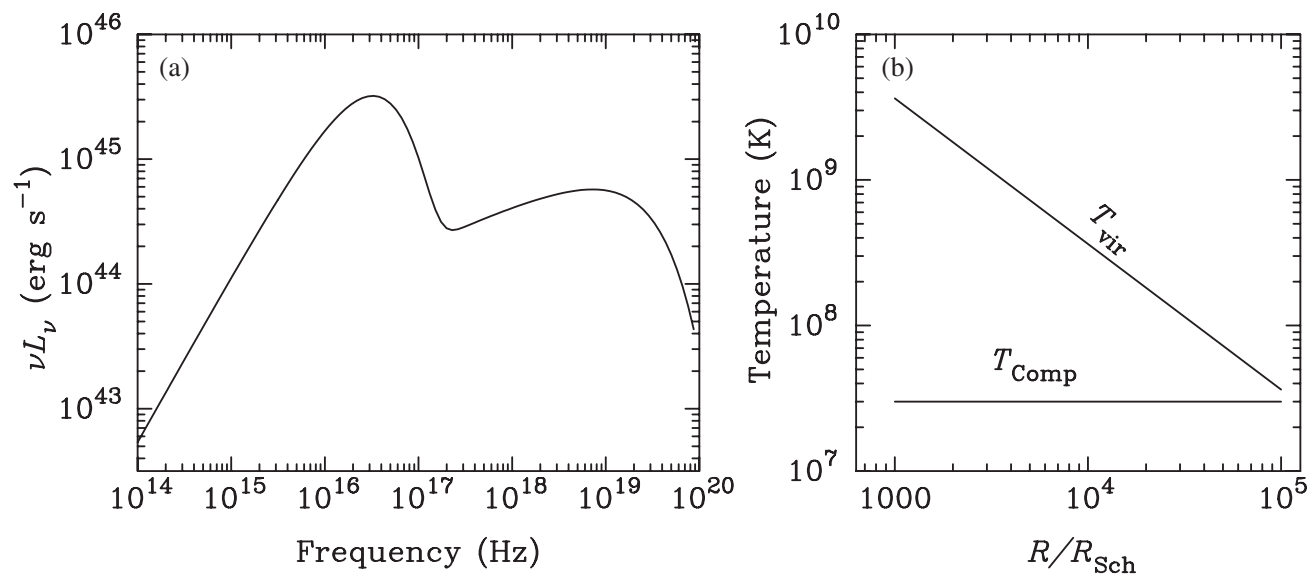

Figure 2. (a) SED from accretion disk with corona. We use the emergent spectrum $F_{v} \propto v^{1 / 3}$ for optical-soft X-rays from the Shakura \& Sunyaev (1973) disk. The spectrum from soft to hard X-rays is a power law with a cutoff. The bolometric luminosity is $10^{46} \mathrm{erg} \mathrm{s}^{-1}$. (b) The Compton temperature $\left(T_{\mathrm{Comp}} \sim 3 \times 10^{3} \mathrm{~K}\right.$ from the SED given by panel (a)) in the surroundings around the SMBHs, compared with the virial temperature. Over most of the region within $1 \mathrm{pc}, T_{\mathrm{Comp}}$ is lower than $T_{\text {vir }}$, implying that all the Compton gas is bound by the SMBH potential.

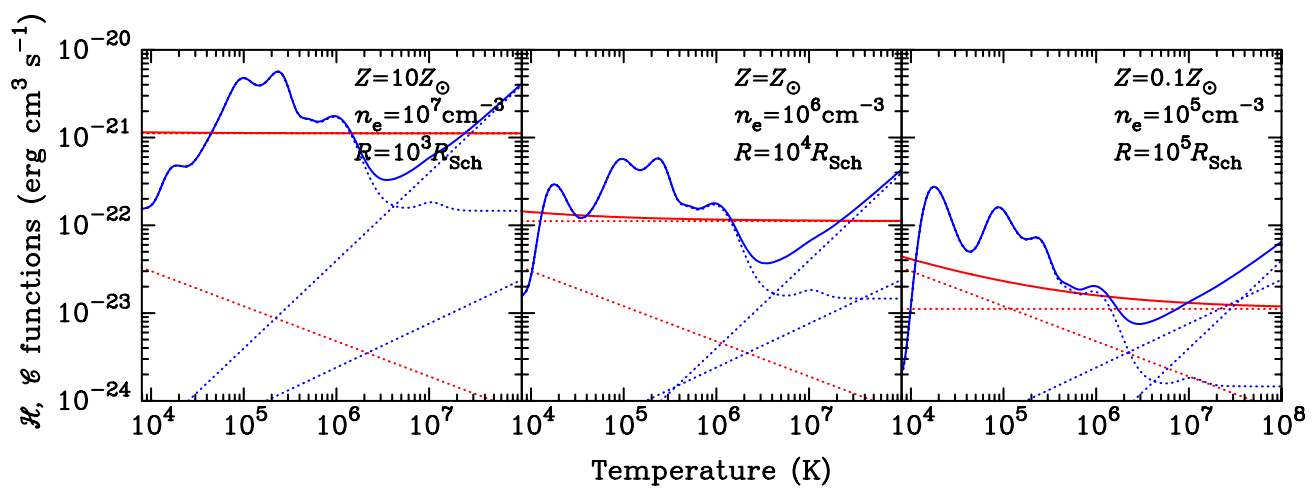

Figure 3. Heating and cooling functions vs. temperature and metallicity at different radii. The blue dotted lines are cooling functions $\left(\mathscr{C}_{\text {comp }}, \mathscr{C}_{\text {ff }}\right.$, and $\left.\mathscr{C}_{\text {line }}\right)$ and the red dotted lines are heating functions $\left(\mathscr{H}_{\mathrm{Comp}}\right.$ and $\left.\mathscr{H}_{\mathrm{ph}}\right)$. The red and blue solid lines are the total heating and cooling functions, respectively. We set the metallicity $Z=10,1,0.1 Z_{\odot}$ at different radii, and typical densities are consistent with results shown by Figure 6, respectively.

constant pressure occurs when $\partial \mathscr{L} / \partial T>0$, since the heating rate rises faster than the cooling rate, determining the fate of the $\mathrm{CAS}$ that has arisen from the SF disk. Figure 3 shows the heating and cooling functions versus temperature at different radii and metallicity. We set $M_{\bullet}=10^{8} M_{\odot}$, bolometric luminosity $L_{\mathrm{Bol}}=10^{46} \mathrm{erg} \mathrm{s}^{-1}$ and the SED of the photoionizing source is from Figure 2.

\subsection{Heated Atmosphere: Geometry}

The warm skin above the SF disk is inevitably heated by the central engine, producing a new static equilibrium. The heated and expanded skin is referred to as the CAS because it has the Compton temperature, $T_{\text {Comp }} \sim 10^{7} \mathrm{~K}$ (see Section 2.2.3). Its height is

$$
\frac{H_{\mathrm{CAS}}}{R} \approx 0.23 t_{\mathrm{C}}^{1 / 2} r_{4}^{1 / 2},
$$

where $t_{\mathrm{C}}=T_{\text {Comp }} / 3 \times 10^{7} \mathrm{~K}$. The higher the Compton temperature, the thicker the CAS. Since the CAS has angular momentum taken from the SF disk, it forms a flattened disk around the axis perpendicular to the SF disk. As we discuss below, cold clouds are born through the thermal instability in the CAS, but they do not always follow the dynamics of the CAS. Once the cold clouds are formed from the CAS, their dynamics and fates are governed by the SMBH gravity, radiation pressure from the central engine, and the friction of the CAS. Depending

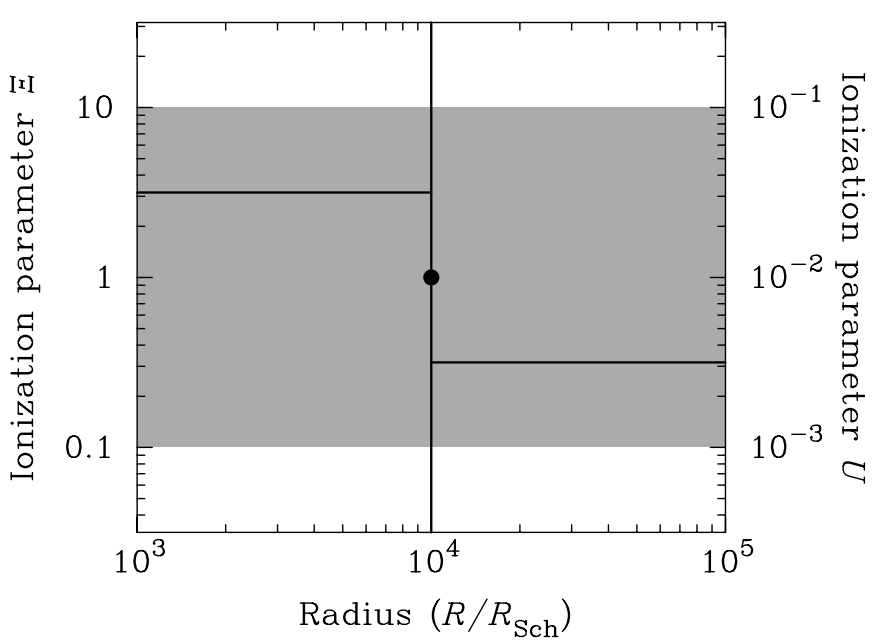

Figure 4. Ionization parameter $\Xi$. We simply divided broad-line regions into two parts according to the ionization parameter. We use the SED to connect the two different ionization parameter $U=10^{-2} \Xi$. The shaded part is the possible regime for clouds emitting lines. The $U$ is usually used to distinguish LIL and HIL regions. The left part is the HIL regions whereas the right part is the LIL regions. The $\sim 10^{4} R_{\mathrm{Sch}}$ is the boundary for the LIL and HIL regions.

on their column density and metallicity, cold clouds are either blown away as cloud outflows driven by the radiation pressure, or sink backward onto the SF disk, or are destroyed by the 
dynamical friction of the CAS and then recycle back into the CAS.

We would like to point out that the Compton temperature is determined by the spectral energy distribution (SED) which depends on the Eddington ratios (Wang et al. 2004). This implies that the BLR geometry might relate to the Eddington ratios, namely, the properties of broad emission lines correlate with the Eddington ratios. This is indeed true in large samples of quasars (Marziani et al. 2009), where the eigenvector 1 spectra depend on the Eddington ratio. Finally, the mass ratio of the Compton gas converting into cold clouds remains a free parameter in the present model. A self-consistent model could resolve this problem.

\subsection{Thermal Equilibrium and Gas Supply}

For a typical luminous QSO with continuum luminosity $L_{1450}=10^{44} \mathrm{erg} \mathrm{s}^{-1} \AA^{-1}$, the total mass needed to provide the emission lines seen from the full BLR is of order $10^{3} M_{\odot}$ (Baldwin et al. 2003a). This estimate includes neutral material in the interiors of optically thick clouds, and we use it here for the total mass of the LIL clouds, which we designate $M_{\mathrm{LIL}}$. For the case of the optically thin HIL clouds, we use just the zones within the clouds which produce C IV emission, which would correspond to $M_{\mathrm{HIL}}=3 M_{\odot}$ for the same continuum luminosity. Following Peterson (1997), we round this down to $M_{\mathrm{HIL}} \sim 1 M_{\odot}$.

The timescales for supplying the HIL and LIL masses are given by

$$
t_{\mathrm{HIL}}=\frac{M_{\mathrm{HIL}}}{\dot{M}_{\mathrm{diff}}}, t_{\mathrm{LIL}}=\frac{M_{\mathrm{LIL}}}{\dot{M}_{\mathrm{diff}}} .
$$

We find that $t_{\mathrm{HIL}} \sim 10 \mathrm{yr}$ for typical $M_{\mathrm{HIL}} \sim 1 M_{\odot}$ and $\dot{M}_{\mathrm{diff}} \sim$ $0.1 M_{\odot} \mathrm{yr}^{-1}\left(R=10^{3}-10^{4} R_{\mathrm{Sch}}\right)$, and $t_{\mathrm{LIL}} \sim 10^{2}-10^{3} \mathrm{yr}$ for $M_{\mathrm{LIL}} \sim 10^{2}-10^{3} M_{\odot}$ and $\dot{M}_{\text {diff }} \sim 1 M_{\odot} \mathrm{yr}^{-1}(R=$ $\left.10^{4}-10^{5} R_{\mathrm{Sch}}\right)$. It generally follows that $t_{\mathrm{HIL}}>t_{\mathrm{Comp}}$ and $t_{\mathrm{LIL}}>$ $t_{\text {Comp }}$. This means that all the supplied gas will be efficiently heated to the Compton temperature until the two timescales are equal. The CAS keeps a quasi-static equilibrium state so that we use a linear analysis to treat its thermal instability, which depends on the equilibrium.

\subsection{Fates of the Clouds}

Once clouds have formed, they are subject to destruction by various dynamical processes provided the CAS is sufficiently dense (see Mathews \& Blumenthal 1977; Krolik et al. 1981; Mathews 1986 for details of these destruction processes). If they are not destroyed instantly, they may undergo one of three different fates depending on their properties and the CAS density. If the clouds' column density is small enough, radiation pressure may blow them away from the region where they were born, forming outflows Boroson (2005) and increasing the metallicity in narrow-line regions (NLRs). This process simply decreases the total mass of the atmosphere. The second possibility is for the clouds to sink to the SF disk since they are too heavy to be supported by the buoyancy and radiation pressure (as shown in Section 3.3.2). This also simply decreases the total mass of the atmosphere. The third possible fate is for a cycle between the CAS and the clouds (Krolik 1988). The clouds are destroyed by the dynamic interaction with the CAS and return mass to the CAS, which then forms new clouds. This opens the way to an eventual collapse of the CAS. The mass cycle does not decrease the total mass of the atmosphere, while at the same time the continuous supply from the SF disk steadily increases the total mass of the CAS. If then at some point the mass stored in the clouds is rapidly released into the atmosphere through dynamical destruction, the optical depth of the atmosphere is suddenly amplified, making the irradiation insufficient and the cooling of the CAS very efficient. This induces the CAS to catastrophically collapse onto the SF disk. This process drives episodic appearance of the BLRs, at least in some parts. We will discuss these complicated processes in Section 4.

Those optically thin clouds formed in the innermost regions are almost instantly blown away by the radiation pressure. This might be related to the formation of NLRs (J.-M. Wang et al. 2011 , in preparation). Evidence for this is the tight correlation between the BLR and the NLR metallicities (J.-M. Wang et al. 2011 , in preparation). The acceleration of the clouds in this region is discussed in Section 4.2.

\section{THE COMPTON ATMOSPHERE: THE BIRTHPLACE OF COLD CLOUDS}

Once the supplied gas is exposed to the central engine, it will be heated up to the Compton temperature and will become a rotating flattened disk of Compton gas. We stress that the continuous injection into the atmosphere from the SF disk leads to a complicated evolution of the atmosphere. The CAS is undergoing heating by the accretion disk and radiative cooling, and also is carrying the Keplerian angular momentum from the SF disk. The resulting gas flows are reflected in the initial motions of the cold clouds that form from the CAS, and thus are reflected in the observed profiles of broad emission lines.

\subsection{The Accumulation of Gas in an Evolving Compton Atmosphere \\ 3.1.1. Mass Budget}

We first consider the mass budget of the CAS. For simplicity, we assume the cold clouds which form from the CAS all have the same mass and size unless we point out the difference in the LIL and HIL.

The CAS evolution can generally be divided into the four phases illustrated by Figure 5: phase I: accumulation of the atmosphere is simply governed by the injection from the SF disk; phase II: formation and pile-up of clouds are driven by the thermal instability along with significant downward spiraling of clouds to the SF disk; phase III: the atmosphere is so dense that sinking clouds are destroyed by dynamical friction, forming a cycle between clouds and atmosphere, and phase IV: the dense atmosphere is not supported by the gas pressure, giving rise to a collapse of the atmosphere into the SF disk.

Phase I continues until the time $t_{1}$ when the line cooling dominates over the Compton cooling. During this phase, we have

$$
M_{\mathrm{CAS}}(t)=\int_{0}^{t\left(\leqslant t_{1}\right)} \dot{M}_{\mathrm{inj}} d t .
$$

At $t_{1}$, the CAS enters phase II. Cold clouds form and separate from the CAS atmosphere. During phase II, some clouds sink to the SF disk and we have

$$
\dot{M}_{\mathrm{CAS}}+\dot{N}_{\mathrm{c}} m_{c}=\dot{M}_{\mathrm{inj}},
$$

where $\dot{N}_{c}$ is the birth rates of clouds and $m_{c}$ is the mass of each cloud. Since the CAS is not yet very dense, the damping can be neglected in this phase. The sinking velocity of clouds can be estimated by $v_{\text {sink }}=v_{\mathrm{K}}\left(H_{\mathrm{CAS}} / R\right)=H_{\mathrm{CAS}} \Omega_{\mathrm{K}}$ with a timescale 

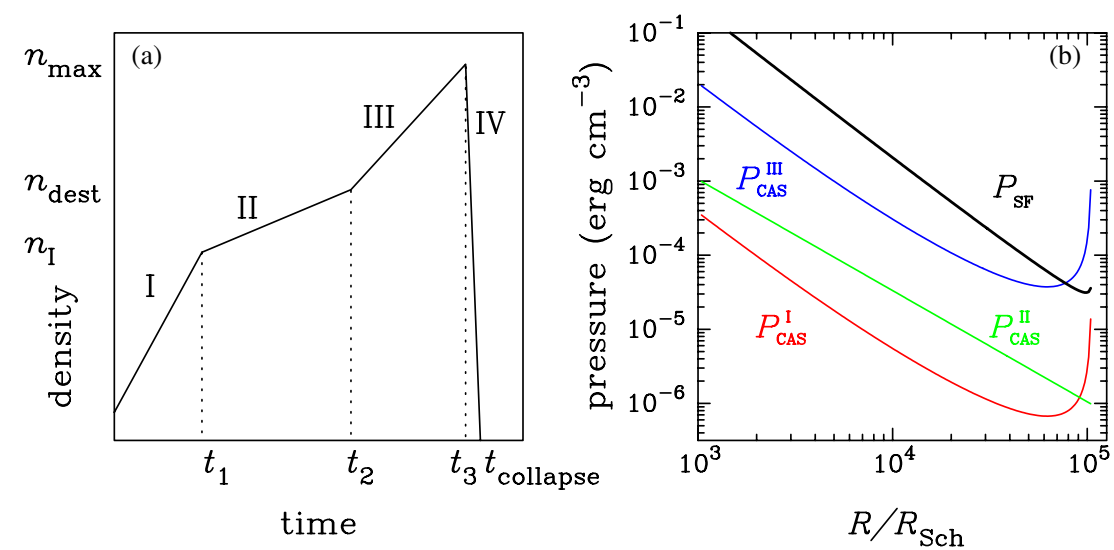

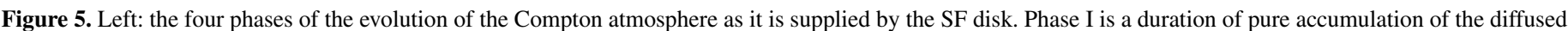

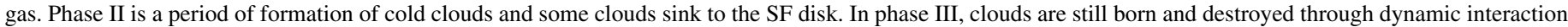

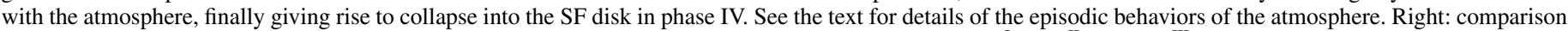

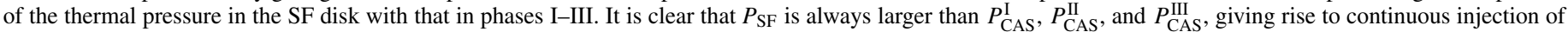
the warm gas into the BLR. The time in the left panel is not shown to scale.

of $t_{\text {sink }}=H_{\mathrm{CAS}} / v_{\text {sink }}=\Omega_{\mathrm{K}}^{-1}$, where $v_{K}$ is the Keplerian rotation velocity. This means that clouds will sink to the SF disk roughly within a Keplerian timescale. It should be noted that this simple estimation does not include the influence of the CAS friction. The sinking timescale could be longer then.

The CAS comes into phase III when it becomes sufficiently dense that dynamical friction destroys the clouds before they can sink all the way to the SF disk, leading to return of cloud material to the atmosphere. We now have

$$
\dot{N}_{c} m_{c}+\dot{M}_{\mathrm{CAS}}=\dot{M}_{\mathrm{inj}}+\dot{N}_{\mathrm{des}} m_{c},
$$

and $\dot{M}_{\text {sink }} \ll \dot{M}_{\text {des }}$, where $\dot{M}_{\text {sink }}=\dot{N}_{\text {sink }} m_{c}$. Since mass continues to be injected from the SF disk, the CAS will become denser and denser until its temperature balance becomes dominated by line cooling. The catastrophe is then triggered, leading to a collapse of the CAS onto the SF disk. The collapse timescale $\left(t_{\text {collapse }}\right)$ is determined by the maximum of the sinking timescale $\left(t_{\text {sink }}\right)$ and cooling timescale $\left(t_{\text {cool }}\right)$, namely,

$$
t_{\text {collapse }}=\max \left(t_{\text {sink }}, t_{\text {cool }}\right) \text {. }
$$

In the following sections, we will determine the critical times and the corresponding densities.

There is a necessary condition for continuous injection from the SF disk into the CAS, namely, the gas pressure in the $\mathrm{SF}$ disk $\left(P_{\mathrm{SF}}\right)$ should be higher than that of the CAS $\left(P_{\mathrm{CAS}}\right)$. Otherwise, the injection will stop if $P_{\mathrm{SF}} \leqslant P_{\mathrm{CAS}}$. These pressures are compared in Figure 4(b), and it is seen that $P_{\mathrm{SF}}>P_{\mathrm{CAS}}$ throughout phases I-III. This guarantees the continuous injection to the CAS from the SF disk.

\subsubsection{Critical Times and Densities}

For a fully ionized Compton gas, the radiative acceleration ${ }^{6}$ is $a_{\mathrm{rad}}=\ell_{\text {Edd }} g$, where $g=G M_{\bullet} / R^{2}$ is the gravitational acceleration. Before line cooling dominates, radiation pressure acting on the ions is mainly through Thompson scattering by electrons. Provided the radiation luminosity of the SMBH is subEddington, the Compton gas is bound by the SMBH potential

\footnotetext{
6 Even for a super-Eddington accreting SMBH, the radiative luminosity is slightly lower than the Eddington luminosity due to photon trapping effects (Wang \& Zhou 1999), and the Compton gas is still bound. This could happen in narrow-line Seyfert 1 galaxies with super-Eddington accretion rates.
}

since its thermal temperature is lower than the virial temperature. The Compton gas will accumulate until it becomes partially ionized. At that point the radiation pressure will be enhanced by line absorption, and the partially ionized gas will be blown away if the radiation pressure is strong enough. There are critical densities of the CAS governing its thermal states in light of the cooling and heating. We discuss the case with $M_{\bullet}=10^{8} M_{\odot}$ and $L_{\text {bol }}=3.0 \times 10^{45} \ell_{0.3} M_{8} \mathrm{erg} \mathrm{s}^{-1}$ to illustrate the evolution of the CAS.

During phase I, the CAS receives gas through thermal diffusion from the SF disk and is cooled mainly by the Compton cooling until the CAS density is high enough so that line cooling dominates in the formed clouds. During phase I, the largest size of a perturbation cannot exceed the vertical height of the CAS given by Equation (15) for a thermal instability which develops as described below by Equation (57). This gives a lower limit of the CAS density as $n_{\min } \sim 10^{4} \mathrm{~cm}^{-3}$. However, cold clouds cannot be formed because their collapse timescale will be much longer than the cooling timescale, and so the thermal perturbation is removed by the dynamics (see Equation (59), below). Therefore, pure accumulation without formation of clouds happens in phase I. Accumulation continues until clouds form, and the CAS enters phase II. This transition from phase I to II is determined by the time at which line cooling in clouds dominates the Compton cooling. The Compton cooling function is given by $\mathscr{C}_{\text {Comp }} \approx 1.8 \times 10^{-23} r_{3}^{-2} M_{8}^{-1} \ell_{0.3} t_{\mathrm{C}} n_{8}^{-1} \mathrm{erg} \mathrm{cm}^{3} \mathrm{~s}^{-1}$. When the CAS density exceeds $n_{\mathrm{I}}$, for a plasma with temperature $10^{5} \mathrm{~K} \leqslant T_{e} \leqslant 10^{7} \mathrm{~K}$, the CAS begins to form clouds. With $\mathscr{C}_{\text {line }} \approx 10^{-21} Z_{0} \operatorname{erg~cm}^{3} \mathrm{~s}^{-1}$ from Figure 3, we have

$$
n_{\mathrm{I}}=1.8 \times 10^{6} M_{8}^{-1} \ell_{0.3} t_{\mathrm{C}} Z_{0}^{-1} r_{3}^{-2} \mathrm{~cm}^{-3} .
$$

The total mass of the CAS is given by the integration over the entire CAS. We have

$$
M_{\mathrm{CAS}}^{\mathrm{I}}=25.3 M_{8}^{2} \ell_{0.3} t_{\mathrm{C}}^{3 / 2} Z_{0}^{-1} r_{5}^{3 / 2} M_{\odot},
$$

where $r_{5}=R / 10^{5} R_{\mathrm{Sch}}$ is the outer boundary of the BLR, and the time is roughly given by

$$
t_{1}=\frac{M_{\mathrm{CAS}}^{\mathrm{I}}}{\dot{M}_{\mathrm{inj}}}=25.3 M_{8}^{2} \ell_{0.3} t_{\mathrm{C}}^{3 / 2} Z_{0}^{-1} r_{5}^{3 / 2} \dot{M}_{\mathrm{inj}, 0}^{-1} \mathrm{yr}
$$

where $\dot{M}_{\text {inj, } 0}=\dot{M}_{\text {inj }} / 1.0 M_{\odot} \mathrm{yr}^{-1}$ is the diffusion rate given by Equation (3). During phase II, once clouds have formed, they 
will spiral down to the SF disk with a sink velocity estimated by $v_{\text {sink }}=v_{\mathrm{K}}(H / R)$, where $v_{K}$ is the Keplerian rotation velocity. This estimate is based on fact that the cloud motions are mainly controlled by the vertical gravity of the SMBH. We have the sink timescale $t_{\text {sink }}=H / v_{\text {sink }}=\Omega_{\mathrm{K}}^{-1}$, which is just the Keplerian one.

There will be a dynamical interaction due to the velocity difference between the clouds and the CAS. This eventually can destroy the clouds and return their material to the CAS, constituting a mass cycle. The destruction timescale is given by $t_{\text {dest }}=3.4 \times 10^{7} \Xi^{-1 / 2} N_{22}\left(t_{\mathrm{C}} T_{4}\right)^{1 / 4}\left(n_{e} T_{e}\right)_{14}^{-1} \Delta v_{500}^{-1} \mathrm{~s}$, where $\Delta v_{500}=\Delta v / 500 \mathrm{~km} \mathrm{~s}^{-1}$ is the velocity difference (Krolik 1988). Setting $t_{\text {sink }}=t_{\text {dest }}$, we have

$$
n_{\text {dest }}=2.5 \times 10^{6} \Xi^{-1 / 2} N_{22} t_{\mathrm{C}}^{-3 / 4} T_{4}^{1 / 4} M_{8}^{-1} \Delta v_{500}^{-1} r_{3}^{-3 / 2} \mathrm{~cm}^{-3},
$$

where $N_{22}=N_{\mathrm{cl}} / 10^{22} \mathrm{~cm}^{-2}$ is the column density of clouds. Ferland et al. (2009) show that the typical column density of Fe II clouds is of order $10^{24} \mathrm{~cm}^{-2}$. We then have, at the point where the cloud destruction takes over and mass cycling begins, the CAS mass

$$
M_{\mathrm{CAS}}^{\mathrm{II}}=2.6 \times 10^{2} \Xi^{-1 / 2} N_{22}\left(T_{4} / t_{\mathrm{C}}\right)^{1 / 4} \Delta v_{500}^{-1} r_{5}^{2} M_{8}^{2} M_{\odot},
$$

corresponding to the time $t_{2}$

$t_{2}=\frac{M_{\mathrm{CAS}}^{\mathrm{II}}}{\dot{M}_{\mathrm{inj}}}=2.6 \times 10^{2} \Xi^{-1 / 2} N_{22}\left(T_{4} / t_{\mathrm{C}}\right)^{1 / 4} \Delta v_{500}^{-1} r_{5}^{2} M_{8}^{2} \dot{M}_{\mathrm{inj}, 0}^{-1} \mathrm{yr}$.

Since the formation of cold clouds decreases the gas pressure in the CAS, $P_{\mathrm{SF}}>P_{\mathrm{CAS}}$ drives the continuous injection into the CAS, entering phase III. However, the start of the mass cycle between the clouds and the Compton gas in the CAS will cause an increase in the gas pressure in the CAS. The CAS reaches a steady state with a balance between production and destruction of clouds, entering phase III.

Once mass begins cycling back and forth between the clouds and the CAS, there is no longer a loss of gas back onto the SF disk, and the CAS density will increase more rapidly in response to the injection from the SF disk. This increase will eventually cause the CAS to enter phase IV, where rather than Compton cooling the entire CAS is cooling mainly through emission lines from $\mathrm{H}$-like $\mathrm{Fe}$ ions. In such a case, the cooling function is approximated by $\mathscr{C}_{\mathrm{Fe}^{+25}}=2.0 \times 10^{-23} Z_{0} \mathrm{erg} \mathrm{cm}^{3} \mathrm{~s}^{-1}$, where $t_{\mathrm{C}}=T / T_{\text {Comp }}$ and $T_{\text {Comp }}=3 \times 10^{7} \mathrm{~K}$. Setting $\mathscr{C}_{\text {Comp }}=\mathscr{C}_{\text {line }}$, we have as the critical density for the start of phase IV (the collapse phase)

$$
n_{\max } \approx 1.0 \times 10^{8} M_{8}^{-1} \ell_{0.3} t_{\mathrm{C}} Z_{0}^{-1} r_{3}^{-2} \mathrm{~cm}^{-3},
$$

with a scale height of $H_{\text {CAS }}$ given by Equation (15). The corresponding mass is roughly obtained through integrating the regions with the density profile (Equation (27)), giving

$$
M_{\mathrm{CAS}}^{\mathrm{III}} \approx 1.3 \times 10^{3} M_{8}^{2} \ell_{0.3} t_{\mathrm{C}}^{3 / 2} Z_{0}^{-1} r_{5}^{3 / 2} M_{\odot},
$$

and the time is roughly

$$
t_{3}=\frac{M_{\mathrm{CAS}}^{\mathrm{III}}}{\dot{M}_{\mathrm{inj}}}=1.3 \times 10^{3} M_{8}^{2} \ell_{0.3} t_{\mathrm{C}}^{3 / 2} Z_{0}^{-1} \dot{M}_{\mathrm{inj}, 0}^{-1} r_{5}^{3 / 2} \mathrm{yr} .
$$

Since the entire CAS is cooling with a timescale (see Equation (62)) much shorter than the injection timescale, a direct collapse of the CAS including the clouds is inevitable within timescale

$$
t_{\text {collapse }} \approx t_{\text {sink }} \approx \frac{R}{v_{\mathrm{K}}}=45.1 M_{8} r_{4}^{3 / 2} \mathrm{yr} .
$$

Following such a collapse, the continued injection from the SF disk drives the BLR to undergo episodic reappearances during the AGN lifetime.

In Figure 5(b), we compare the SF disk pressure with the CAS pressure during phases I-III. We use $\left(P_{\text {CAS }}^{\mathrm{I}}, P_{\mathrm{CAS}}^{\mathrm{II}}, P_{\mathrm{CAS}}^{\mathrm{III}}\right)=$ $\left(n_{\mathrm{I}}, n_{\text {dest }}, n_{\text {max }}\right) k T_{\text {Comp }}$ for the three phases, respectively. The metallicity appearing in the critical density is given by the results in W11. We find that the SF disk pressure is always higher than the CAS pressure. This simply indicates that the SF disk continuously injects material into the CAS. This is a necessary condition for the episodic appearance of the BLR. On the other hand, the injection timescale is much longer than the dynamical timescale, allowing us to approximate the CAS by a static state at any time except during phase IV.

It is interesting to find that the accumulated mass of the BLR agrees well with the observational estimates given above in Section 2.4. Our model predicts that BLR-I (the HIL gas) and BLR-II (the LIL gas) are spatially separated, and have $\sim 1 M_{\odot}$ and $\sim 300 M_{\odot}$, respectively. The estimates based on the observed emission-line strengths are of order $M_{\mathrm{HIL}} \sim 1 M_{\odot}$ and $M_{\mathrm{LIL}} \sim 10^{3} M_{\odot}$. This provides independent support for the present model of the BLR formation.

We find that the maximum CAS mass is very sensitive to the SMBH mass, but also to the Eddington ratio, implying the dependence of the BLR mass on the SMBH mass and accretion rate through the star formation rate. For narrow-line Seyfert 1 galaxies with $M_{\bullet} \sim 10^{6.5} M_{\odot}$, the relevant timescales could be significantly reduced, but relying on accretion rates. We will consider the case of narrow-line Seyfert 1 galaxies in the future.

It not expected that significant soft X-rays are emitted from the CAS. The emission from free-free cooling is estimated to be $L_{\mathrm{SX}}=4 \pi \int n_{e}^{2} \mathscr{C}_{\mathrm{ff}} R H_{\mathrm{CAS}} d R$. The phase I CAS emits $L_{\mathrm{SX}} \sim 2.0 \times 10^{39} \mathrm{erg} \mathrm{s}^{-1}$ and the Thompson scattering depth is $\tau_{\text {es }} \approx 0.4$. This soft X-ray emission can be totally neglected compared with the bolometric luminosity. We should point out that most of the injected mass $M_{\mathrm{CAS}}$ will be rapidly converted into clouds after phase I, but the CAS gas will emit soft Xrays during the formation of clouds. In phase II, the injected mass is efficiently converted into clouds, and the luminosity from the CAS cooling is given by $L_{\mathrm{SX}} \sim k T_{\mathrm{C}} \dot{M}_{\mathrm{inj}} / m_{p} \approx$ $1.7 \times 10^{41} \mathrm{erg} \mathrm{s}^{-1}$. This again is not significant.

We note that the continuous injection could be stopped if pressure of the CAS is higher than the pressure of the hot gas in the SF disk. This could happen if the star formation rates are not high enough in the case of some LLAGNs. Also thermal conduction between the CAS and the warm gas in the SF disk could be important. The underlying physics will be discussed separately for the LLAGNs. We should point out here that the above four phases are just an outline of the complicated evolution of the atmosphere. The four phases of the atmosphere are radius-dependent, forming the LIL regions and HIL regions at different times. We will investigate the four phases in further detail in the following sections.

\subsection{Thermal Instability}

It has been long known that the partially ionized gas exposed to a radiation field can undergo thermal instability (Field 1965). 
It develops when $\partial \mathscr{L} / \partial T<0$. Formation of BLRs driven by the thermal instability has been extensively studied (Krolik et al. 1981; Beltrametti 1981; Shlosman et al. 1985; Krolik 1988; or more recently Pittard et al. 2003 for the more complicated case of winds). Basic results from these studies show that a twophase medium will be formed through the thermal instability. However, most of results do not directly apply to the present case because the authors did not include the role of the angular momentum of the gas that is undergoing the thermal instability. This plays a key role in determining the geometry of the resulting BLR. Furthermore, all these studies neglect the role of metallicity in the condensation of the clouds. Beltrametti (1981) demonstrated that cold clouds are formed at a $1 \mathrm{pc}$ scale for metal-free gas, clearly larger than $R_{\mathrm{BLR}} \approx 0.1 L_{46}^{1 / 2}$ pc obtained from reverberation mapping, where $L_{46}=L_{\mathrm{Bol}} / 10^{46} \mathrm{erg} \mathrm{s}^{-1}$ is the bolometric luminosity. We argue that the inconsistency is due to the metal-free assumption (Beltrametti 1981; Shlosman et al. 1985). A detailed study of the thermal instability of the atmosphere including the angular momentum and metallicity is one of main goals of the present paper. We start from the basic equations of the atmosphere.

\subsubsection{Governing Equations}

The atmosphere is described by a velocity field $\vec{v}$, density $\rho$, temperature $T$, and pressure $p$, which are a function of radius, in the potential $(\Phi)$ of the SMBH gravity. The SF disk as the lower boundary of the atmosphere continuously injects warm gas with a function $\mathcal{S}$. Thermal conduction in the atmosphere is included in the energy equation. The atmosphere can be descried by a series of equations: (1) the continuity equation

$$
\frac{\partial \rho}{\partial t}+\nabla \cdot(\rho \vec{v})=0
$$

(2) momentum conservation

$$
\frac{\partial \vec{v}}{\partial t}+(\vec{v} \cdot \nabla) \vec{v}=-\frac{1}{\rho} \nabla p-\nabla \Phi
$$

and (3) energy conservation

$$
\begin{aligned}
\frac{p}{\gamma-1}\left(\frac{\partial}{\partial t}+\vec{v} \cdot \nabla\right) \ln \left(p \rho^{-\gamma}\right)= & \nabla \cdot\left(\kappa_{\mathrm{S}} T^{2.5} \nabla T\right) \\
& +\left(\frac{\rho}{\mu \mathrm{m}_{p}}\right)^{2} \mathscr{L}(\rho, T),
\end{aligned}
$$

where $\mathscr{L}(\rho, T)=\mathscr{H}-\mathscr{C}$ is the net heating function, $\mathscr{H}$ and $\mathscr{C}$ are heating and cooling functions, respectively, and here $\gamma$ is the ratio of principle specific heats of the gas. We assume the system has a cylindrical asymmetry so that all terms of $\partial / \partial \phi=0$. In cylindrical coordinates, the above equations can be re-cast in three components

$$
\begin{gathered}
\frac{\partial \rho}{\partial t}+\frac{1}{R} \frac{\partial}{\partial R}\left(R \rho v_{R}\right)+\frac{\partial}{\partial z}\left(\rho v_{z}\right)=0 \\
\frac{\partial v_{R}}{\partial t}+v_{R} \frac{\partial v_{R}}{\partial R}+v_{z} \frac{\partial v_{R}}{\partial z}=-\frac{1}{\rho} \frac{\partial p}{\partial R}-\frac{\partial \Phi}{\partial R}+\frac{v_{\phi}^{2}}{R}, \\
\frac{\partial v_{z}}{\partial t}+v_{R} \frac{\partial v_{z}}{\partial R}+v_{z} \frac{\partial v_{z}}{\partial z}=-\frac{1}{\rho} \frac{\partial p}{\partial z}-\frac{\partial \Phi}{\partial z}
\end{gathered}
$$

$$
\begin{gathered}
\frac{\partial v_{\phi}}{\partial t}+v_{R} \frac{\partial v_{\phi}}{\partial R}+v_{z} \frac{\partial v_{\phi}}{\partial z}=-\frac{v_{R} v_{\phi}}{R} . \\
\frac{p}{\gamma-1}\left[\frac{\partial}{\partial t}+v_{R} \frac{\partial}{\partial R}+v_{z} \frac{\partial}{\partial z}\right] \ln \left(p \rho^{-\gamma}\right) \\
=\frac{1}{R} \frac{\partial}{\partial R}\left(R \kappa_{\mathrm{S}} T^{5 / 2} \frac{\partial T}{\partial R}\right)+\frac{\partial}{\partial z}\left(\kappa_{\mathrm{S}} T^{5 / 2} \frac{\partial T}{\partial z}\right) \\
+\left(\frac{\rho}{\mu \mathrm{m}_{p}}\right)^{2} \mathscr{L}(\rho, T) .
\end{gathered}
$$

The potential of the SMBH gravity is given by

$$
\Phi(R, z)=-\frac{G M_{\bullet}}{\left(R^{2}+z^{2}\right)^{1 / 2}},
$$

where $z$ is height from the SF disk plane. Here the threedimensional velocity components are $v_{R}, v_{\phi}$, and $v_{z}$.

The dynamical timescale of the CAS gas is much shorter than the timescale of gas supply. This allows us to assume that the gas maintains hydrostatic and thermal equilibrium at any time and treat the gas supply as a small perturbation. In order to proceed to study the global instability of the atmosphere, we simply assume that the injection function $\mathcal{S} \approx 0$ and obtain the solution for the barotropic gas. We emphasize that the injection is so important for the BLR that it may drive a transient appearance of a BLR in some AGNs.

\subsubsection{Equilibrium Configurations}

The detailed thermal behavior of the atmosphere is sensitive to the gas temperature and density distribution, so the conclusions drawn in the cases of clusters and galaxies cannot be a priori extended to the BLR atmosphere. The equilibrium condition of the gas is important not only because the thermal instability depends on the equilibrium configuration, but also because the clouds formed due to the instability follow the density distribution in the equilibrium state. When the Compton cooling and heating reach equilibrium, the gas becomes a Compton gas with a uniform temperature. We give the equilibrium states of the barotropic gas. For a static state of the atmosphere, $v_{R}=v_{z}=0$.

The Poincaré-Wavre theorem states that the surfaces of constant pressure and constant density coincide if and only if $\partial \Omega / \partial z=0$. Although the assumption of a barotropic gas is simplified, it illustrates the main features as a good approximation. The rotating atmosphere in cylindrical coordinates can be described by the following equations re-cast from Equations (34)-(37)

$$
\begin{gathered}
\frac{1}{\rho_{0}} \frac{\partial p_{0}}{\partial R}+\frac{\partial \Phi}{\partial R}-\frac{v_{\phi}^{2}}{R}=0, \\
\frac{1}{\rho_{0}} \frac{\partial p_{0}}{\partial z}+\frac{\partial \Phi}{\partial z}=0 .
\end{gathered}
$$

For a barotropic gas, it follows that $p_{0}=K_{0} \rho_{0}^{\gamma}$ and the angular velocity $\Omega$ is a function only of $R$. The analytical solution of Equations (40) and (41) is given by $\gamma p_{0} /(\gamma-1) \rho_{0}+\Phi=$ $\int \Omega^{2} R d R$ for $\gamma \neq 1$. For a presumed rotation of the atmosphere as

$$
\Omega=\omega_{0} \Omega_{0}\left(\frac{R}{R_{\text {out }}}\right)^{-q},
$$



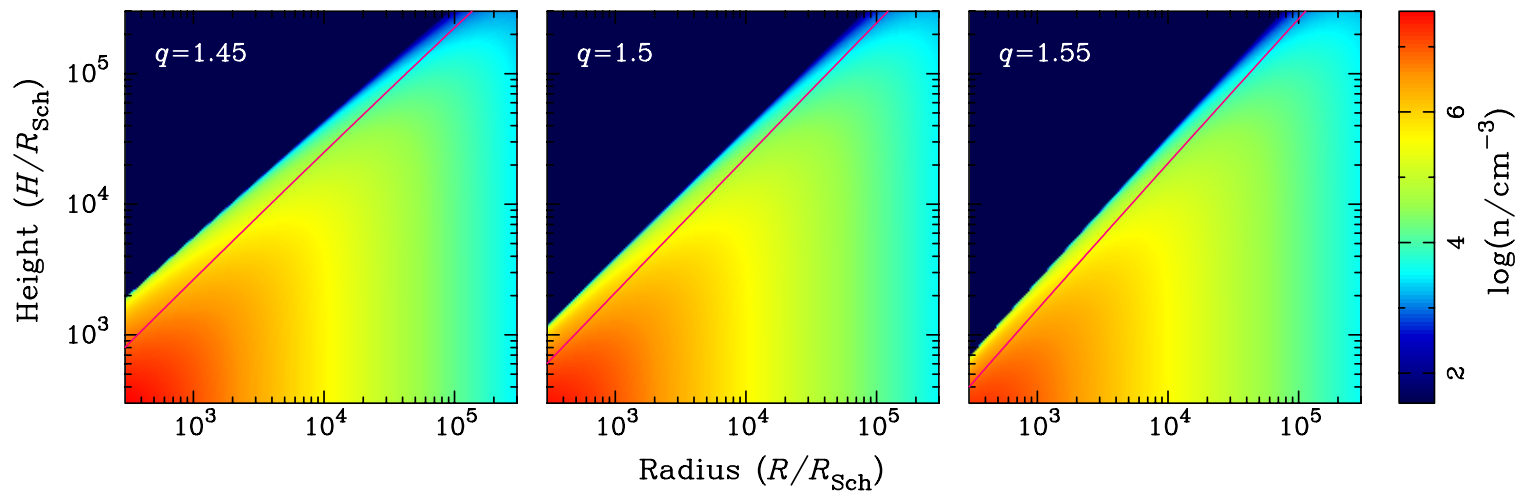

Figure 6. Isodensity profiles of the barotropic model of the atmosphere. We use $n=1.4, \omega_{0}=0.5$, and $\rho_{* 0}=5.8 \times 10^{-20} \mathrm{~g} \mathrm{~cm}^{-3}$, but change the index of angular momentum $(q)$, and the constant $C_{0}=0$ for each case. The total mass of the CAS is $M_{\mathrm{CAS}}=1.3 \times 10^{3} M_{\odot}$. The red line is the boundary of the atmosphere beyond which clouds are not able to form.

where $\Omega_{0}=\left(G M_{\bullet} / R_{\text {out }}^{3}\right)^{1 / 2}$ is a constant, and $\omega_{0}$ is a constant for correction of the angular momentum, we have the equilibrium solution

$$
\frac{p_{0}}{\rho_{0}}=\frac{\gamma-1}{\gamma}\left[C_{0}-\Phi+\frac{\omega_{0}^{2} \Omega_{0}^{2}}{2-2 q}\left(\frac{R}{R_{\text {out }}}\right)^{-2 q} R^{2}\right],
$$

where $C_{0}$ is a constant to be determined by the total mass of the atmosphere. Appendix A gives the solutions for $\gamma=1$. The zero-pressure surface is given by

$$
\Phi-\frac{\omega_{0}^{2} \Omega_{0}^{2}}{2-2 q}\left(\frac{R}{R_{\text {out }}}\right)^{-2 q} R^{2}=C_{0} .
$$

It is convenient to take the constant $\Omega_{0}=c / \sqrt{2} R_{\mathrm{Sch}} r_{\mathrm{out}}^{3 / 2}$, which is the Keplerian rotation velocity. Equation (44) then can be re-cast as

$$
\frac{\omega_{0}^{2} r_{\mathrm{out}}^{2 q-3}}{2(q-1)} \frac{1}{r^{2(q-1)}}-\frac{1}{\left(r^{2}+h^{2}\right)^{1 / 2}}=C_{0}^{\prime},
$$

where $r=R / R_{\mathrm{Sch}}, h=z / R_{\mathrm{Sch}}$, and $C_{0}^{\prime}=2 C_{0} / c^{2}$. The properties of the zero-pressure surface are fully determined by the index $q$ and $C_{0}^{\prime}$. The case of a constant specific angular momentum has been discussed by Papaloizou \& Pringle (1984). In the present case, we assume that the atmosphere retains the Keplerian angular momentum carried from the SF disk. Actually, the atmosphere will re-redistribute the angular momentum through viscosity leading to a change the density distribution. This redistribution could be a self-regulated process since the total mass could be a few $10^{3} M_{\odot}$ injected from the SF disk with a mass rate of $\sim 1 M_{\odot} \mathrm{yr}^{-1}$. The main goal of the present paper is to explore the solution of the atmosphere with a presumed angular momentum distribution, in order to obtain information about cloud formation in the BLRs. A self-consistent solution for the dynamics of the atmosphere will be carried out in a separate paper.

Since the inner radius of the SF disk is set at the selfgravitating radius, the atmosphere is assumed to be confined within the radii between $R_{\mathrm{SG}}$ and $R_{\text {out }}$. For the barotropic gas, the isodensity and zero-pressure surfaces overlap. Employing the relation of $p_{0}=K_{0} \rho_{0}^{\gamma}$, we have the density distribution

$$
\rho_{0}(r, h)=\rho_{*}\left[\frac{\omega_{0}^{2} r_{\mathrm{out}}^{2 q-3}}{4(1-q)} \frac{1}{r^{2(q-1)}}+\frac{1}{2\left(r^{2}+h^{2}\right)^{1 / 2}}+C_{0}\right]^{n},
$$

where we take $K_{0}=k T_{\text {Comp }} \rho_{* 0}^{1-\gamma} / m_{p}$ for convenience, $\rho_{*}=$ $\rho_{* 0}[\gamma /(\gamma-1)]^{-1 /(\gamma-1)}\left(c_{s} / c\right)^{2 /(1-\gamma)}, \rho_{* 0}$ is a constant, and the constant $C_{0}$ is then given by the total mass of the atmosphere

$$
M_{\mathrm{CAS}}=\iint \rho_{0}(R, z) 2 \pi R d R d z .
$$

Here, $M_{\mathrm{CAS}}$ is the total mass supplied by the thermal diffusion, which is a time-dependent parameter in the system.

Figure 6 shows solutions of the barotropic models for different distributions of angular momentum. These results are found to be very sensitive to the distribution of angular momentum in the atmosphere (Equation (42)). We concentrate on the sub-Keplerian cases since the diffusing gas only carries the Keplerian angular momentum, unless there is a mechanism to drive the atmosphere to rotate with super-Keplerian velocity. For the atmosphere with a sub-Keplerian rotation, the atmosphere shrinks from its initial size (i.e., $R<R_{\text {out }}$ ), increasing the density of the atmosphere. The geometry of the atmosphere is actually quite thick, $H / R \sim 1$, but getting thinner with increasing $q$. This indicates that the BLR would be geometrically thick. The atmosphere has a sharp upper boundary as shown by Figure 6. The radial distribution of the density decreases with radius. The rough estimate given by Equation (15) is consistent with the barotropic model. However, the barotropic approximation should be revisited in future work on the atmosphere. The effect of including redistribution of angular momentum through viscosity (and even heating by the atmosphere) is worth exploring.

\subsubsection{Perturbation Equations and Dispersion Relation}

We assume that the equilibrium gas is axisymmetric so that all of the terms $\partial / \partial \phi=0$, and that the fluid rotates differentially with $\Omega=\Omega(R, z)$. The velocity of the gas with density $\left(\rho_{0}\right)$ is given by $\left(v_{R}^{0}, v_{\phi}^{0}, v_{z}^{0}\right)$ for hydrostatic and thermal equilibrium. The velocity perturbations are $\left(v_{R}^{1}, v_{\phi}^{1}, v_{z}^{1}\right)$ and the density is $\rho_{1}$. Supposing an axisymmetric perturbation happens in a form of $F=F_{0}+F_{1} \exp \left(-i \omega t+i k_{R} R+i k_{z} z\right)$, where $F_{0}$ is the parameter value at the equilibrium and the perturbation $\left|F_{1}\right| \ll F_{0}$, the linearized equations can be written as

$$
\begin{gathered}
i k_{R} \rho_{0} v_{R}^{1}+i k_{z} \rho_{0} v_{z}^{1}-i \hat{\omega} \rho_{1}=0, \\
-i \hat{\omega} \rho_{0} v_{R}^{1}-2 \Omega \rho_{0} v_{\phi}^{1}-\mathcal{A}_{p R} c_{0}^{2} \rho_{1}+i k_{R} p_{1}=0, \\
-i \hat{\omega} \rho_{0} v_{z}^{1}-\mathcal{A}_{p z} c_{0}^{2}+i k_{z} p_{1}=0,
\end{gathered}
$$




$$
\left(\Omega+\Omega_{R}\right) v_{R}^{1}+\omega_{z} v_{z}^{1}-i \hat{\omega} v_{\phi}^{1}=0
$$

and

$$
\begin{aligned}
& \left(\mathcal{A}_{p R}-\gamma \mathcal{A}_{\rho R}\right) v_{R}^{1}+\left(\mathcal{A}_{p z}-\gamma \mathcal{A}_{\rho z}\right) v_{z}^{1} \\
& \quad+\left(i \hat{\omega}-\omega_{\mathrm{d}}\right) \gamma \rho_{0}^{-1} \rho_{1}-i \hat{\omega} p_{0}^{-1} p_{1}=0,
\end{aligned}
$$

where $\hat{\omega}=\omega-\left(k_{R} v_{R}^{0}+k_{z} v_{z}^{0}\right), \omega_{\mathrm{d}}=\omega_{c}+\omega_{\mathrm{th}}, \Omega_{R}=\partial(\Omega R) / \partial R$,

$$
\omega_{c}=\frac{\gamma-1}{\gamma} \frac{\kappa\left(k_{R}^{2}+k_{z}^{2}\right) T_{0}^{7 / 2}}{p_{0}}
$$

and

$$
\omega_{\mathrm{th}}=\frac{\gamma-1}{\gamma}\left(\frac{\rho_{0}}{\mu \mathrm{m}_{p}}\right)^{2} \frac{\xi_{0} \mathscr{L}_{0}}{p_{0}}
$$

where $\mathscr{L}_{0}=\mathscr{L}\left(\rho_{0}, T_{0}\right)$ and $\xi_{0}=\partial \ln \mathscr{L}_{0} / \ln \rho_{0}-$ $\partial \ln \mathscr{L}_{0} / \partial \ln T_{0}+2$ at the equilibrium configuration given by Equation (43).

We use the approximation of short-wavelength and lowfrequency perturbations, with $k_{1} R \sim k_{2} R \gg 1$. This approximation is valid in cases where the size of cold clouds is much smaller than the coordinate $R$, meaning that $R k_{1} \gg 1$ and $z k_{2} \gg 1$. The case of $R k_{1} \gg 1$ is guaranteed since the BLR radius is much larger than the size of the clouds. However, $z k_{2} \gg 1$ indicates that the vertical regions should be much larger than the size of clouds, implying that the approximation is only valid for the quite geometrically thick disk.

Appendix B gives details of the derivation of the dispersion relation. For barotropic gas, we have the dispersion relation given by Equation (B27)

$$
\left(\hat{n}+\omega_{\mathrm{d}}\right)\left(\hat{n}^{2}+\omega_{\text {rot }}^{2}\right)=0 .
$$

We have

$$
\hat{n}=-\omega_{\mathrm{c}}-\omega_{\mathrm{th}} \text { or } \hat{n}^{2}=-\omega_{\text {rot }}^{2} .
$$

For a Keplerian rotation, we have $\ell \propto R^{1 / 2}$, we always have $\omega_{\text {rot }}>0$, namely, $\hat{n}^{2}<0$, implying that the system is stable. Only if $\omega_{\mathrm{d}}<0$ does thermal instability develop, yielding

$$
\kappa_{\mathrm{S}}\left(k_{R}^{2}+k_{z}^{2}\right) T_{0}^{7 / 2}+\mathscr{L}_{0} \xi_{0}\left(\frac{\rho_{0}}{\mu \mathrm{m}_{p}}\right)^{2}<0 .
$$

This inequality determines the regions of the thermal instability. Given the equilibrium configuration, the minimum wavelength of the perturbation can be found from Equation (57). Perturbations shorter than the minimum length will be removed by thermal conduction and no instability can be developed. Since the perturbation length is much less than the disk height scale, we consider the simple case of $k_{R} \sim k_{z}=2 \pi / \lambda_{\mathrm{TI}}$, namely, spherical clouds where $\lambda_{\mathrm{TI}}$ is the size of the perturbation which is not able to be smeared by the thermal conduction. We have

$\lambda_{\mathrm{TI}} \geqslant \frac{2 \sqrt{2} \pi \kappa_{\mathrm{S}}^{1 / 2} T_{0}^{7 / 4}}{\left|\mathscr{L}_{0} \xi_{0}\right|^{1 / 2}}\left(\frac{\mu \mathrm{m}_{p}}{\rho_{0}}\right) \approx 1.7 \times 10^{14} T_{7}^{2.4} n_{6}^{-1} Z_{0}^{-1 / 2} \mathrm{~cm}$,

where $\xi_{0}=2.78, \mu=0.67$, and $\mathscr{L}_{0}=7.77 \times$ $10^{-22} Z_{0} \mathrm{erg} \mathrm{cm}^{3} \mathrm{~s}^{-1}$ for typical parameter values $T_{0}=10^{7} T_{7} \mathrm{~K}$ and $\rho_{0}=1.67 \times 10^{-18} n_{6} \mathrm{~g} \mathrm{~cm}^{-3}$. The dependence on metallicity originates from the line cooling function. For the line-coolingdominated case, we roughly have $\mathscr{L} \propto T^{-1.3} Z$ and $\xi_{0}$ is not sensitive to the temperature and density. The dependence on temperature results from the thermal conduction and the line cooling function. The size of the clouds depends on the thermal conductivity $\kappa_{\mathrm{S}}$, which determines the minimum cloud size. For the CAS, the temperature is nearly homogeneous in space. On the other hand, the maximum wavelength set by $\lambda_{\max }$ is so long that sound waves cannot cross it in a cooling time, so that compression will not follow cooling and growth is suppressed (Shlosman et al. 1985). This yields

$$
\lambda_{\max } \leqslant c_{s} t_{\mathrm{cool}}^{\mathrm{ff}} \approx 5.2 \times 10^{15} T_{7} n_{6}^{-1} \mathrm{~cm},
$$

where $t_{\mathrm{cool}}^{\mathrm{ff}}$ is the free-free cooling timescale and $c_{s}$ is the sound speed of the hot phase. The column density of the clouds will be $N_{\mathrm{H}}=n_{e} \lambda_{\mathrm{TI}}$,

$$
N_{\mathrm{H}}^{0}=\frac{2 \sqrt{2} \pi \mu \kappa_{\mathrm{S}}^{1 / 2} T_{0}^{7 / 4}}{\left|\mathscr{L}_{0} \xi_{0}\right|^{1 / 2}} \approx 1.7 \times 10^{20} T_{7}^{2.4} Z_{0}^{-1 / 2} \mathrm{~cm}^{-2} .
$$

It should be noted that this is the initial column density of clouds. Once clouds form, they efficiently shrink until they reach a new thermal equilibrium with a temperature $\left(T_{\mathrm{cl}}\right)$ and get a new column density.

Note that for a CAS with a density lower than $10^{4} \mathrm{~cm}^{-3}$, the minimum length of the perturbation will be larger than the height of the CAS. This means that the thermal instability is not able to develop and formation of clouds is forbidden. This gives the lower limit of the CAS density for cloud formation.

\subsection{Formation of Clouds}

\subsubsection{Initial Phase of Formation}

Once thermal instability is triggered, the perturbed gas will undergo contraction until clouds finally form and then maintain a pressure balance with their surrounding medium. These processes are very complicated (see the review by Meerson 1996; or recent papers of Iwasaki \& Tsuribe 2008, 2009, but which only deal with a one-dimensional medium). Krolik (1988) investigated cloud formation in an inflow and outflow under the condition of broken radiative equilibrium, where the convergence or divergence of the inflows and outflows enhance or diminish the cooling in clouds, giving rise to only algebraic growth of clouds rather than exponential. The present CAS is in static equilibrium, so the context of cloud formation is different from the case described by Krolik (1988). Clouds are born swirling around in the CAS. The initial phase of contraction is driven by the free-free cooling on a timescale of

$$
t_{\mathrm{ff}} \approx\left\{\begin{array}{l}
0.58 n_{7}^{-1} T_{7}^{1 / 2} \operatorname{yr}\left(R=10^{3} R_{\mathrm{Sch}}\right), \\
5.8 n_{6}^{-1} T_{7}^{1 / 2} \mathrm{yr}\left(R=10^{4} R_{\mathrm{Sch}}\right) .
\end{array}\right.
$$

We find that this timescale is still smaller than the Keplerian one, $t_{\mathrm{Kep}}=2 \pi / \Omega_{\mathrm{K}} \approx 9.0 r_{3}^{3 / 2} M_{8}=283.0 r_{4}^{3 / 2} M_{8}$ yr. Due to the high metallicity, the subsequent cooling through line emission is much more efficient than bremsstrahlung cooling.

\subsubsection{Final States of Cold Clouds}

When the density of the Compton gas exceeds $n_{c}^{\mathrm{ff}}$ after the initial phase, for a plasma with temperature $10^{4} \mathrm{~K} \leqslant T_{e} \leqslant 10^{7} \mathrm{~K}$, the cooling is mainly through elements $\mathrm{C}, \mathrm{He}, \mathrm{O}, \mathrm{Mg}$, and $\mathrm{Fe}$. The cooling timescale is given by $t_{\text {cool }} \approx n_{e} k T_{e} / \Lambda_{\text {cool }}\left(n_{e}, T_{e}, Z\right)$, where the cooling function is approximated by $\Lambda\left(n_{e}, T_{e}, Z\right)=$ $n^{2} \mathscr{C}_{\text {line }}=2.0 \times 10^{-10} n_{6}^{2} T_{6}^{-1.3} Z_{0} \operatorname{erg~s}^{-1} \mathrm{~cm}^{-3}$ (see Equation 
(25) in W11). We then have as the formation timescale of cold clouds

$$
t_{\text {cloud }} \sim t_{\text {cool }} \approx\left\{\begin{array}{l}
0.05 n_{7}^{-1} T_{7}^{2.3} Z_{1}^{-1} \operatorname{yr}\left(R=10^{3} R_{\mathrm{Sch}}\right), \\
5.0 n_{6}^{-1} T_{7}^{2.3} Z_{0}^{-1} \operatorname{yr}\left(R=10^{4} R_{\mathrm{Sch}}\right)
\end{array}\right.
$$

which is much shorter than the diffusion time of the Compton gas and the Keplerian rotation period.

Following onset of the thermal instability, cold clouds form in the timescale given by Equation (62), within one Keplerian rotation. This allows us to reasonably assume that the cold clouds have the same angular momentum as gas diffused from the SF disk since the specific angular momentum of the gas approximately remains constant (neglecting the radiation pressure for the Compton gas).

The temperature of cold clouds can be determined from the new thermal equilibrium condition $\mathscr{H}_{\text {Comp }}+\mathscr{H}_{\text {ph }}=\mathscr{C}_{\text {ff }}+\mathscr{C}_{\text {Comp }}+$ $\mathscr{C}_{\text {line }}(\Xi)$, while density can be determined from the pressure equilibrium with the CAS as given by $n_{\mathrm{cl}} T_{\mathrm{cl}}=n_{\mathrm{CAS}} T_{\mathrm{CAS}}$, yielding $n_{\mathrm{cl}}=\left(T_{\mathrm{CAS}} / T_{\mathrm{cl}}\right) n_{\mathrm{CAS}}$ and $T_{\mathrm{cl}} \sim 10^{4} \mathrm{~K}$. The initial mass of a single cloud is given by $\Delta m_{c}=4 \pi \lambda_{\mathrm{TI}}^{3} n_{\mathrm{CAS}} / 3$. Considering mass conservation of clouds, we have $\lambda_{\mathrm{cl}}^{3} n_{\mathrm{cl}}=$ $\lambda_{\mathrm{TI}}^{3} n_{\mathrm{CAS}}$, where $\lambda_{\mathrm{cl}}$ and $n_{\mathrm{cl}}$ are the final radius and density of formed clouds. Since pressure equilibrium holds, we have $n_{\mathrm{cl}} T_{\mathrm{cl}}=n_{\mathrm{CAS}} T_{\mathrm{CAS}}$. The final column density of cold clouds is obtained from

$$
N_{\mathrm{H}}=\left(\frac{T_{\mathrm{CAS}}}{T_{\mathrm{cl}}}\right)^{2 / 3} N_{\mathrm{H}}^{0} \approx 1.7 \times 10^{22} T_{7}^{3.1} T_{4}^{-2 / 3} Z_{0}^{-0.5} \mathrm{~cm}^{-2}
$$

where $T_{4}=T_{\mathrm{cl}} / 10^{4} \mathrm{~K}$. We note that the final temperature depends on metallicity as shown in Figure 3. It is likely that clouds born in the outer BLR are different from ones formed in the innermost regions of the BLR. Due to the metallicity gradient, this naturally causes the difference between high- and low-ionization regions. The evolution of clouds depends on the metallicity of the clouds.

Here we neglect the dependence of the line cooling and photoionization heating on the ionization parameter. Since the cooling is strongly dependent on the metallicity, the final column density of clouds will be different from that given in Equation (63) because the new thermal equilibrium temperature for $10 Z_{\odot}$ will be of $10^{5} \mathrm{~K}$ (from Figure 3 ). Future papers in this series will treat the clouds in a more self-consistent manner using photoionization models and will study the dependence of cloud properties on the metallicity.

\subsection{Global Structure of the BLR \\ 3.4.1. Spatial Distribution of Clouds}

The CAS density profile is described by Equation (46), which in turn determines the spatial distribution of the cloud's formation rate in the torus since $\delta \rho \propto \rho_{0}$, where $\delta \rho$ is the overdensity of the cloud compared with its surroundings. The spatial distribution of cold clouds generally follows the hot medium, but with the constraint that clouds can only form in locations where the cooling timescale is shorter than the sound crossing timescale. Furthermore, for an initial perturbation with length $\Delta R$, a necessary condition for cloud formation is $\Delta R_{\min }=$ $1.7 \times 10^{14} T_{7}^{2.4} n_{6}^{-1} Z_{0}^{-1 / 2} \mathrm{~cm}$ should be less than the local height of the CAS. We write this condition as $\Delta R_{\min }=\alpha_{\mathrm{cl}} H_{\mathrm{CAS}}$, where $\alpha_{\mathrm{cl}}$ is a (poorly known) fraction of the CAS height below which clouds can form. We have a critical density for cloud formation:

$$
n_{\min } \geqslant 4.2 \times 10^{4} \alpha_{\mathrm{cl}, 0.1}^{-1} M_{8}^{-1} T_{7}^{1.9} r_{4}^{-3 / 2} Z_{0}^{-1 / 2},
$$

where $\alpha_{\mathrm{cl}, 0.1}=\alpha_{\mathrm{cl}} / 0.1$. The parameter $\alpha_{\mathrm{cl}}^{-1} \sim H_{\mathrm{CAS}} / \Delta R_{\mathrm{cl}}$ determines the number of clouds along the vertical direction in the CAS, where $\Delta R_{\mathrm{cl}}$ is the size of the formed clouds. Clouds can only form where the density is greater than $n_{\min }$. The isodensity plot in Figure 6 shows the geometry of the BLR with the CAS density indicated by the color. Once clouds have formed, their fate will depend on their column density.

\subsubsection{Dynamics of Clouds}

Following their formation, the clouds are decoupled from the atmosphere and move in response to the gas pressure gradient, the radiation pressure, and the SMBH gravitational potential. The vertical support from the gas pressure gradient can be estimated by $F_{\text {gas }} \sim\left(\Delta R_{c} / H_{\mathrm{CAS}}\right) P_{\mathrm{gas}} \Delta R^{2}=$ $\rho_{\mathrm{CAS}} k T_{\mathrm{Comp}}\left(\Delta R_{c}\right)^{2} / m_{p}=\rho_{\mathrm{CAS}} T_{\mathrm{Comp}}\left(\Delta R_{c}\right)^{3} / H_{\mathrm{CAS}} m_{p}$, which is due to the difference in the gas pressure between the bottom and the top of the clouds, where $m_{p}$ is the proton mass and $k$ is the Boltzmann constant. The gravity in the vertical direction is given by $F_{\mathrm{G}} \sim G M_{\bullet} \rho_{\mathrm{c}}\left(\Delta R_{c}\right)^{3} H / R^{3}$. We find that

$$
\frac{F_{\mathrm{gas}}}{F_{\mathrm{G}}} \sim \frac{\rho_{\mathrm{CAS}}}{\rho_{\mathrm{c}}} \sim 10^{-3},
$$

where we use $H \sim c_{s} / \Omega_{\mathrm{K}}$ and $c_{s}=\left(k T_{\mathrm{Comp}} / m_{p}\right)^{1 / 2}$ and $\rho_{\mathrm{CAS}} / \rho_{c} \sim 10^{-3}$.

The angular momentum carried by the clouds inevitably causes them to spiral downward toward the disk. Since cold clouds form in a timescale shorter than the dynamical or the Keplerian timescales, the angular momentum of cold clouds should follow that of the hot medium. The angular momentum of the hot medium is given by Equation (42). For a given angular momentum value, a cloud's orbit around the SMBH is easily calculated. Detailed dynamics will be derived in a following paper, from which we will compute emission-line profiles which can be compared with observations.

\subsubsection{Spatial Distribution of the Ionization Parameter}

Since the density profile varies as $\rho \propto R^{-1.5}$ as shown in Figure 6 , we have $\Xi \propto R^{\sim-0.5}$. Figure 7 shows the ionization parameter in the CAS calculated from Equation (13). It shows that the vertical $\Xi$-structure is quite homogeneous whereas the radial structure can be roughly divided into an inner high- $\Xi$ and an outer low- $\Xi$ region at the boundary $R_{\text {ion }} \sim 10^{4} R_{\text {Sch. }}$. At that radius, $\Xi$ falls below a value of about $10^{1.2}$. This corresponds to $U \sim 0.15$. Photoionization models show that $U>10^{-1}$ for HIL (C IV) regions whereas $U \sim 10^{-2}$ for LIL $(\mathrm{H} \beta)$ regions. Photoionization models show that $U=1-10$ for HIL (C IV) regions whereas $U=10^{-2}-1$ for LIL ( $\mathrm{H} \beta$ ) regions (e.g., Marziani et al. 2010).

As a brief summary of this section, we have shown that there are four phases of the evolving CAS. This drives an episodic appearance of the BLRs, which can be compared with observations. We have systematically analyzed the thermal instability of the CAS and find that it is generally driving the formation of clouds once the CAS has entered phase II. The key point in the present model is the continuous injection of warm gas supplied by the SF disk. The minimum and maximum size of clouds can be estimated from the instability analysis. The final 

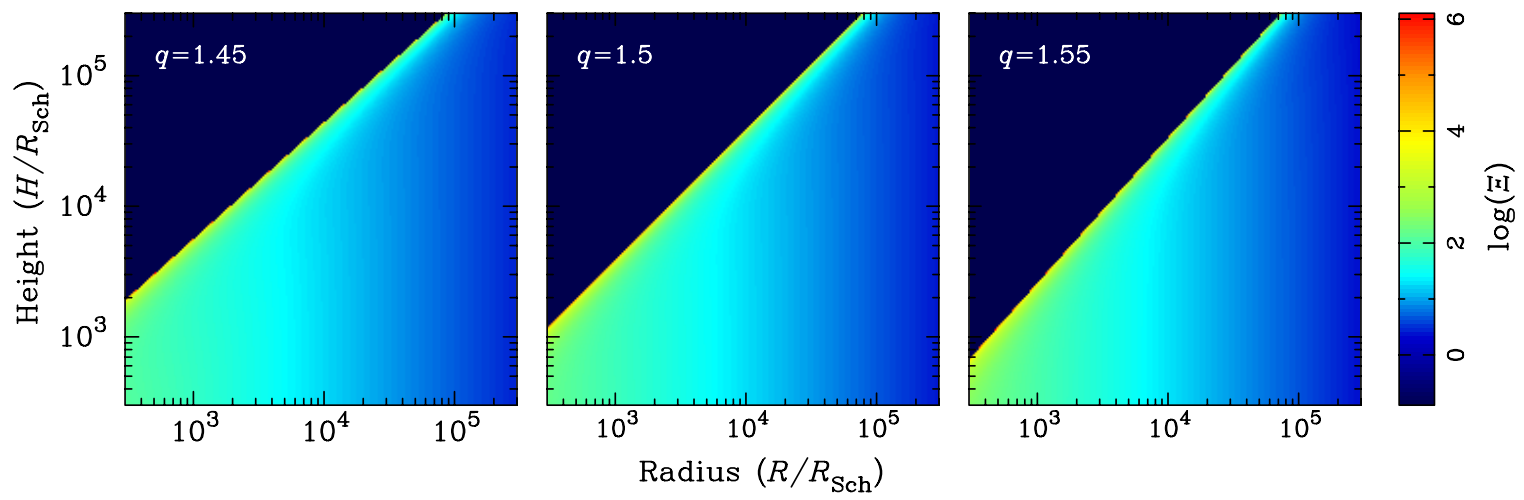

Figure 7. Ionization parameters in the CAS. The three panels correspond to the density profiles shown in Figure 6 . We use $L_{\text {ion }}=10^{45} \mathrm{erg} \mathrm{s}^{-1}$.

column density of clouds has been estimated from the model. Figure 8 sketches the global scenario described by this model.

\section{BROAD-LINE REGIONS}

Since there is a metallicity gradient in the CAS (W11), the properties of clouds formed from that gas also depend on the gradient. Since the thermal equilibrium temperature is higher with higher metallicity (Figure 3), the high-metallicity, highionization clouds in the innermost regions are hotter than the low-metallicity, low-ionization clouds found in the outer part of the CAS. The ionization parameter reads $\Xi=L /\left(4 \pi R^{2} n k T\right) \sim$ $N_{\mathrm{H}}^{-1} T^{-1} \propto T^{-0.3} Z^{0.5}$, implying $\Xi$ is a function of distance of the ionized clouds from the center. We simply divided the CAS into HIL regions and LIL regions at the break-point $\Xi=10^{1.2}$, which occurs at $R_{\text {ion }} \sim 10^{4} R_{\text {Sch }}$ (Figure 7).

The separation between the HIL and LIL regions has been well known since the work of Netzer (1980), Kwan \& Krolik (1979), and Collin-Souffrin et al. (1988). However, these authors only postulated that the two different BLR regions must exist in order to explain the observed properties of broad emission lines. Our present model predicts the existence of the two regions, based on physical arguments, as a natural consequence of the presence of an SF disk. We find that they have spherical and flattened geometry, respectively, separated at about at $\sim 0.1 \mathrm{pc}$.

\subsection{Two-phase Medium}

As we have shown, continuous injection from the SF disk drives the CAS into phase II, and then the thermal instability leads to the CAS becoming a two-phase medium consisting of cold clouds and Compton gas. The filling factor of the evolving BLR can be estimated. The mass of individual clouds is

$$
\Delta m_{c}=\frac{4 \pi}{3} \lambda_{\mathrm{TI}}^{3} n_{\mathrm{CAS}} m_{p}=3.5 \times 10^{-9} \lambda_{14}^{3} n_{6} M_{\odot}
$$

where $\lambda_{14}=\lambda_{\mathrm{TI}} / 10^{14} \mathrm{~cm}$ is the initial length of the perturbations. Considering the fact that the timescale of cloud formation is much shorter than that on which gas is supplied from the SF disk, when the CAS enters phase II the supplied gas is rapidly converted into clouds. The fraction of the mass that is converted into clouds can be determined from the ionization parameter $\Xi$. For a CAS with mass $M_{0}$, the cloud formation stops if $\Xi \geqslant \Xi_{c}$, where $\Xi_{c} \sim 10$ (Krolik et al. 1981), and we have the fraction $f_{c}=M_{0} / n_{\mathrm{h}} V m_{p}-1=\Xi_{c} / \Xi_{0}-1$, where $\Xi_{0}=L / 4 \pi R^{2} c n_{0} k T$ and $n_{0}=M_{0} / V m_{p}$, namely, mass $M_{0}$ with a density $n_{0}$ has the minimum $\Xi$. When $\Xi$ changes by $\Delta \Xi$, the fraction $f_{c}=\Delta \Xi / \Xi$, which will be much less than the unity. Since $t_{\text {sink }} \gg t_{\text {cloud }}$, we have from Equation (18)

$$
\dot{N}_{c} \approx \frac{f_{0.1} \dot{M}_{\mathrm{inj}}}{\Delta m_{c}} \approx 10^{8} f_{0.1} \dot{M}_{\mathrm{inj}, 0} \Delta m_{-9}^{-1} \mathrm{yr}^{-1},
$$

where $f_{0.1}=f_{c} / 0.1, \Delta m_{-9}=\Delta m_{c} / 10^{-9} M_{\odot}$, and the accumulated number of clouds during phase II is given by

$$
\mathscr{N}_{\text {II }} \approx \dot{N}_{c} t_{2}=2.6 \times 10^{10} \dot{N}_{c, 8}\left(\frac{t_{2}}{2.6 \times 10^{2} \mathrm{yr}}\right),
$$

where $\dot{N}_{c, 8}=\dot{N}_{c} / 10^{8} \mathrm{yr}^{-1}$. Such a large number of clouds are enough to explain the smooth profile of the broad emission lines (Arav et al. 1997). The filling factor can be simply estimated as

$$
\mathcal{C}_{\text {II }} \approx \mathscr{N}_{\text {II }}\left(\frac{\lambda_{c}}{R}\right)^{2}\left(\frac{\lambda_{c}}{H}\right) \approx 10^{-6} \mathscr{N}_{10}\left(\frac{\lambda_{13}}{R_{1 \mathrm{pc}}}\right)^{3}\left(\frac{H}{R}\right)_{0.1}^{-1},
$$

where $\mathscr{N}_{10}=\mathscr{N}_{\text {II }} / 10^{10}, \lambda_{13}=\lambda_{\mathrm{cl}} / 10^{13} \mathrm{~cm}$, and $(H / R)_{0.1}=$ $(H / R) / 0.1$ for the CAS. This filling factor is generally consistent with observations of the line luminosity. This expression is important for testing the present model through the filling factor of the BLRs. It indicates that the "age" of the BLR in phases II and III can be represented by the filling factor, which can be estimated from the equivalent widths (EWs) of the emission lines. We note that such a large number of clouds could lead to collisions among them, which could be described by Boltzmann equation (Whittle \& Saslaw 1986). We do not treat this problem in this paper.

During phase II, clouds formed through thermal instability are sinking to the SF disk. The sinking rate is evolving with time. Without detailed calculations of cloud dynamics, it is not trivial to deduce the sinking rate. However, the maximum sinking rate can easily be obtained from

$$
\dot{N}_{\text {sink }} \lesssim \dot{N}_{c} \approx 10^{8} \mathrm{yr}^{-1} .
$$

This rate can be tested from observations of AGNs with redshifted intermediate components of $\mathrm{H} \beta$ (Hu et al. 2008b).

The behavior of the clumpy BLR in phase III can be estimated in a way similar to the above. The BLR is full of cloudlets emitting broad emission lines, but the intermediate component of $\mathrm{H} \beta$ shifts backward toward the rest wavelength until it overlaps with the very broad component, with an observable absence of the redshifted component. We point out that different parts of the BLR may be in different phases at the same time due to the variation of the cloud and hot-phase gas properties as a function of distance from the black hole. 

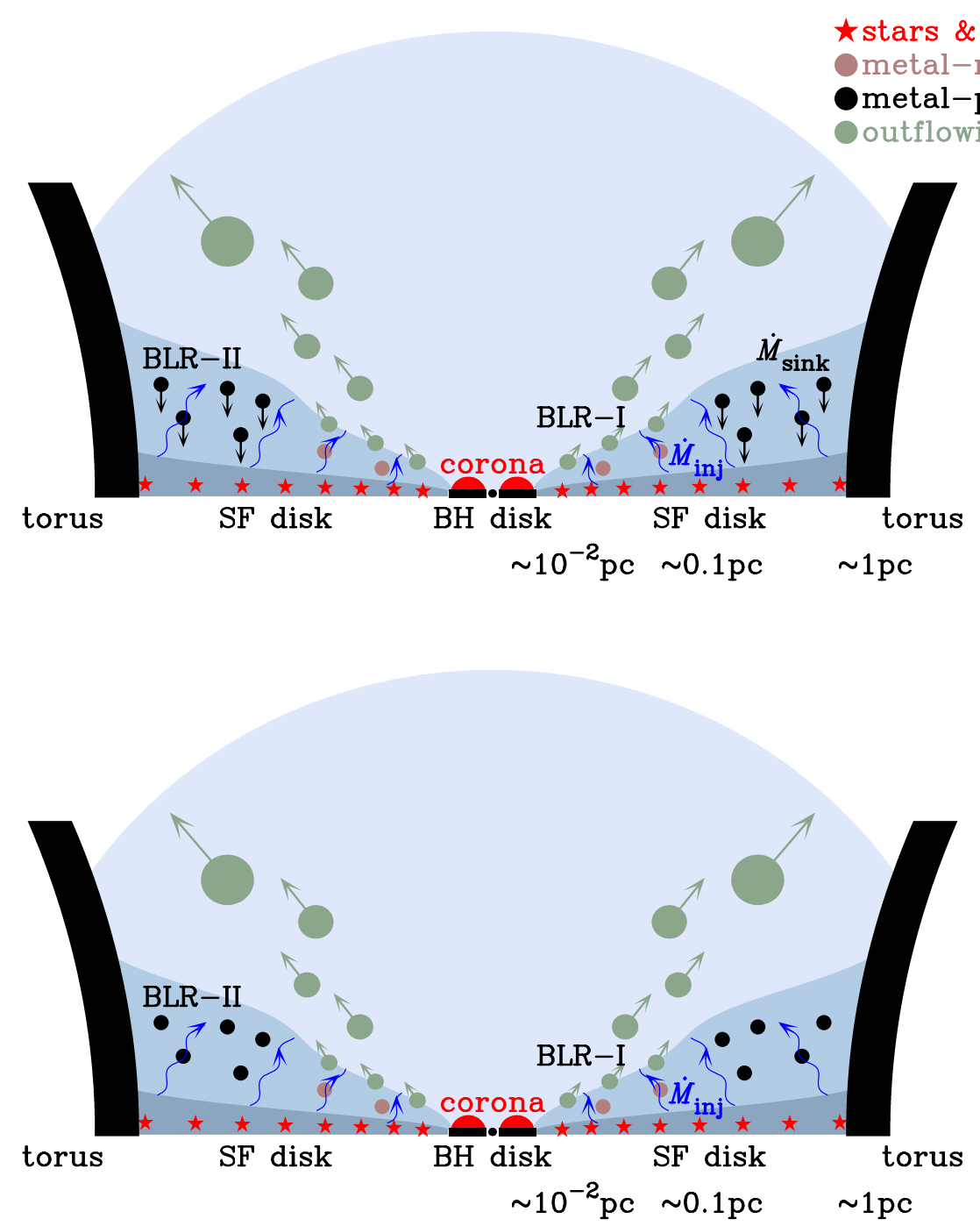

Figure 8. Sketches of the BLR in phase II (upper panel) and phase III (lower panel). In either phase, the BLR extends inward from the inner edge of the dusty torus to the self-gravity radius of the accretion disk. SNexp heating causes gas to be injected from the SF disk at a rate $\dot{M}_{\text {inj }}$. This gas is heated by emission of the SMBH accretion disk to become the Compton gas, and then forms into discrete clouds due to the two-phase instability in the Compton atmosphere. BLR-I is formed from the metal-rich gas that is injected from the inner part of the SF disk and then is pushed outward and upward away from the disk by radiation pressure. BLR-II is formed from the lower-metallicity gas injected from the outer part of the SF disk, which then stays close to the disk surface. In the case of phase II (upper panel), individual clouds form in BLR-II and then sink back down into the SF disk. In phase III (lower panel), these clouds are dynamically destroyed and merge back into the Compton atmosphere before they have time to sink all the way to the SF disk.

\subsection{The High-ionization-line BLR: Outflowing Clouds}

In the presence of the metallicity gradient, the innermost region of the SF disk usually has metallicity $Z \sim 10 Z_{\odot}$ (W11). For a concise discussion, we take the metallicity $Z=10 Z_{\odot}$ in this region. The thermal equilibrium temperature is $T \lesssim$ $6 \times 10^{4} \mathrm{~K}$ (from Figure 3, left panel). The column density of the clouds formed here is about $N_{\mathrm{HIL}}^{\mathrm{H}} \sim 5 \times 10^{21} Z_{1}^{-0.5}$ from Equation (63). This simply indicates that the clouds in the HIL region are optically thin. The gravitational acceleration is given by $g_{\bullet}=G M_{\bullet} / R^{2}=15.0 M_{8}^{-1} r_{3}^{-2} \mathrm{~cm} \mathrm{~s}^{-2}$.

The cold clouds are accelerated by radiation pressure. For optically thin clouds the strength of the radiation pressure depends linearly on the metallicity as $a_{\text {rad }} \propto Z$, but for partially ionized clouds the dependence is approximately $a_{\mathrm{rad}} \propto Z^{0.4}$ (Abbott 1982), where $a_{\text {rad }}$ is the acceleration of radiation due to line absorption. We simply take a linear dependence for optically thin clouds and neglect radiative acceleration for optically thick clouds. The metallicity gradient in the BLR leads to difference of a factor of a few in the radiation force multiplier for the LIL and HIL regions. The acceleration due to radiation pressure of an isotropic field is given by (e.g., Netzer \& Marziani 2010)

$$
\begin{aligned}
a_{\mathrm{rad}}(R) & =\frac{\sigma_{\mathrm{T}} L_{\mathrm{Bol}}}{4 \pi R^{2} c m_{p}} \mathcal{M} \\
& =108.0 \ell_{0.3} \alpha_{0.2} M_{8}^{-1} r_{3}^{-2} Z_{0} N_{22}^{-1} \mathrm{~cm} \mathrm{~s}^{-2},
\end{aligned}
$$

where $N_{22}=N_{\mathrm{H}} / 10^{22} \mathrm{~cm}^{-2}, \mathcal{M}=\left(Z / Z_{\odot}\right) \alpha_{\text {ion }} / \sigma_{\mathrm{T}} N_{\mathrm{H}}$ is the force multiplier, and $\alpha_{\text {ion }}$ is the fraction of the bolometric luminosity absorbed by the clouds. Here we use $\alpha_{0.2}=\alpha_{\text {ion }} / 0.2$, which is absorbed by the gas. We note that this acceleration is comparable with the gravity of SMBHs, but is dominant for $N_{\mathrm{H}}<10^{22} \mathrm{~cm}^{-2}$. The accelerated cloud will escape the potential of the SMBH if it reaches the escape velocity $V_{\mathrm{esc}}=$ $\left(G M_{\bullet} / R\right)^{1 / 2}=6.7 \times 10^{3} r_{3}^{-1 / 2} \mathrm{~km} \mathrm{~s}^{-1}$ for which the timescale is

$$
t_{\mathrm{acc}}=\frac{V_{\mathrm{esc}}}{a_{\mathrm{rad}}}=0.2 M_{8} r_{3}^{3 / 2} \ell_{0.3}^{-1} \alpha_{0.2}^{-1} Z_{0}^{-1} N_{22} \mathrm{yr} .
$$


Table 2

Predicted Properties of Evolving BLRs in a Single Episode

\begin{tabular}{llccc}
\hline \hline Phase & \multicolumn{1}{c}{ Observed Properties } & $\begin{array}{c}\text { Duration } \\
\text { (Years) }\end{array}$ & Appearance & Notes and Ref. \\
\hline I & Bright type II like AGNs without polarized broad lines & 25.3 & $\sim 1.6 \%$ & A few objects \\
II & One VBC and one redshifted IMC and redshifted Fe II & 260.0 & $\sim 16.0 \%$ & H08 \\
III & Overlapped one VBC and one IMC, non-redshifted Fe II & $1.3 \times 10^{3}$ & $\sim 79.7 \%$ & H08 \\
IV & Appearance of the maximum equivalent width of lines & 45.1 & $\sim 1.8 \%$ & $? ? ?$
\end{tabular}

Notes. VBC: very broad components with a typical width of a few $10^{3} \mathrm{~km} \mathrm{~s}^{-1}$, and IMC: intermediate component with a typical width of $\sim 10^{3} \mathrm{~km} \mathrm{~s}^{-1}$. Evolution of AGN BLR from phases I-IV forms a spectral sequence of BLR as indicated by the observed properties. "A few objects" refer to NGC 3660 (Tran 2001); 1ES 1927-654 (Boller et al. 2003); ESO 416-G00211 and PMN J0623-4636 (Gallo et al. 2006); Q2130-431 (Panessa et al. 2009) and two SDSS quasars: J161259.83+421940.3 and J104014.43+474554.8. H08: Hu et al. (2008a, 2008b).

The timescale for the actually escaping is given by $t_{\mathrm{esc}}=$ $R / V_{\text {esc }}=1.4 r_{3}^{3 / 2} M_{8} \mathrm{yr}$, which is comparable with the acceleration timescale. The escape timescale is much shorter than the Keplerian rotation period. Therefore, optically thin clouds are very rapidly blown away by the radiation pressure as soon as they are born.

The HIL-BLR is dominated by optically thin clouds, and hence by the outflowing clouds. The observed C IV and other HILs often show complicated profiles, indicating outflows. The mass rates can be estimated from the supply rates from the SF disk within the HIL regions. The clouds could be transported to the NLRs with a timescale of $t_{\text {tran }}=R / V_{\text {esc }}=$ $1.6 \times 10^{5} R_{1 \mathrm{kpc}} V_{6000}^{-1} \mathrm{yr}$, where $R_{1 \mathrm{kpc}}=R / 1 \mathrm{kpc}$ and $V_{6000}=$ $V_{\text {esc }} / 6000 \mathrm{~km} \mathrm{~s}^{-1}$. During the transportation, the clouds will appear as warm absorbers in soft X-rays, which are observed in many objects.

\subsection{Low-ionization Line BLR: Transient States}

For the LIL-BLR, the ionization states of clouds are relatively lower since they are located further away from the central energy source of the SMBH accretion disk. On the other hand, the metallicity of clouds originating in the outer part of the SF disk is lower than the innermost regions. W11 show that the metallicity is about $0.1 Z_{\odot}$ in this region. When the CAS enters phase II, the new thermal equilibrium of clouds with low metallicity results in a temperature around $10^{4} \mathrm{~K}$ (from Figure 3, right panel). The final column density of the clouds is then $N_{\text {LIL }}^{\mathrm{H}} \sim 5.4 \times 10^{22} Z_{0.1}^{-0.5} \mathrm{~cm}^{-2}$. Such a thick cloud only undergoes negligible radiative acceleration. The dynamics of these clouds is relatively simple in phase II. They are spiraling downward toward the SF disk as shown by Equation (65). The timescale of sinking to the SF disk is $t_{\text {sink }} \sim t_{\mathrm{Kep}}=R / V_{\mathrm{K}}$. These sinking clouds constitute infalling flows, showing redshifted Balmer lines to the observer. Later, after further continuous injection from the SF disk, the CAS becomes so dense that it prevents the clouds from sinking to the SF disk. The CAS then enters phase III. This results in much smaller infall velocities, and the observed Balmer lines shift back toward their rest wavelength. Finally, the LIL regions collapse into the SF disk since the entire CAS becomes dominated by line cooling. These transitions are qualitatively discussed in Section 3.1.

We would like to stress the difference between the mass circulation in phase II and the mass cycle in phase III. The former term refers to the circulation between the CAS and SF disk (i.e., the sinking of clouds back onto the SF disk), leading to a slower growth of the CAS than in phase I. The term "mass cycle," on the other hand, refers to a rapid exchange of mass back and forth between the CAS and the clouds due to the rapid dynamical destruction of clouds as they move through the CAS. This latter cycle drives the CAS to rapidly become sufficiently dense that it enters phase IV, the collapse of the BLRs.

In summary, we have attempted to build up a global scenario of BLRs working from first principles. The key ingredient in this model is the continuous injection of warm gas from the SF disk. This gives rise to the episodic appearance, disappearance, and then the reappearance of the BLRs, with four phases within each episode. The fates of clouds formed in different regions are determined by the metallicity of the gas coming from different parts of the SF disk. Low- and high-ionization regions are then formed naturally. The HIL clouds are blown away by the radiation pressure, transporting metal-rich material to the NLRs. The LIL region undergoes a more complicated evolution, finally resulting in the collapse of the entire CAS. In light of the complexity of the above analytical discussions, it would be worth carrying out numerical simulations to show more details of these phases of BLR formation.

\section{OBSERVATIONAL TESTS OF THE EPISODIC BLR}

The present model makes a number of clear theoretical predictions: (1) the BLRs are transient; (2) the episodes of BLR state transition, driven by the star formation in the self-gravitating disk, are a few thousand years in length, giving rise to a spectral sequence of broad emission lines; (3) the BLRs are separated into HIL and LIL regions, which form a steady gradient of metallicity, with HIL regions having higher metallicity than LIL regions; and (4) there is an intrinsic connection between the BLR and NLR through outflows developed from the HIL regions.

These predictions can be tested observationally. First of all, non-BLR AGNs are a key constituent of the transient BLRs. Here non-BLR AGNs are those that have typical accretion rates $\left(\sim 0.2 L_{\text {Edd }}\right)$, but do not have BLRs. These represent phase I in our model. We have to find them, even though they are relatively rare (only $\sim 10^{-2}$ probability of appearance; see Table 2). Second, we should be able to find and identify QSOs in each of the sequential phases predicted by our model-there should be a spectral sequence of broad emission lines. Third, although the existence of separate HIL and LIL BLRs has been known observationally for many years, we predict that the HIL and LIL regions have metallicity differences arising from the different rates of star formation in different parts of the SF disk. The observed metallicity difference between HIL and 
Table 3

AGN Types in the Unification Scheme

\begin{tabular}{|c|c|c|c|c|}
\hline $\begin{array}{l}\text { Type } \\
\text { (1) }\end{array}$ & $\begin{array}{l}\text { Eddington Ratio } \\
\text { (2) }\end{array}$ & $\begin{array}{c}\text { SMBH Mass }\left(M_{\odot}\right) \\
(3)\end{array}$ & $\begin{array}{c}N_{\mathrm{H}}\left(\mathrm{cm}^{-2}\right) \\
(4)\end{array}$ & $\begin{array}{l}\text { Note and Ref. } \\
\text { (5) }\end{array}$ \\
\hline Seyfert 1 & $\sim 0.25$ & $\sim 10^{7}$ & $\lesssim 10^{22}$ & $\sqrt{ }$ \\
\hline Seyfert 2 & $\sim 0.25$ & $\sim 10^{7}$ & $\gtrsim 10^{22}$ & $\sqrt{ }$ \\
\hline "True" Seyfert 2 & $<10^{-2}$ & $\sim 10^{\wedge} 7$ & $<10^{21}$ & $\sqrt{ }$ \\
\hline Narrow-line Seyfert 1 & $\sim 1$ & $10^{6}-10^{7}$ & $<10^{22}$ & $\sqrt{ }$ \\
\hline Obscured NLS1s & $\sim 1$ & $10^{6}-10^{7}$ & $\gtrsim 10^{22}$ & $\sqrt{ }(\mathrm{ZW06})$ \\
\hline Unabsorbed Seyfert 2 & $\sim 0.25$ & $\sim 10^{7}$ & $\lesssim 10^{22}$ & $\sqrt{ }$ \\
\hline \multicolumn{5}{|c|}{ New types predicted by the present model } \\
\hline Phase-I AGNs & $\sim 0.25$ & $\sim 10^{7}$ & $\lesssim 10^{21}$ & "Panda" AGNs, ? \\
\hline Phase-IV AGNs & $\sim 0.25$ & $\sim 10^{7}$ & $\lesssim 10^{21}$ & Very large EW,? \\
\hline
\end{tabular}

Notes. Columns are (1) types of AGNs (see details in Wang \& Zhang 2007); (2) the averaged Eddington ratio; (3) SMBH mass in units of solar mass; (4) absorbing hydrogen column density; and (5) notes. Nomenclature: NLS1: narrow-line Seyfert 1; ZW06: Zhang \& Wang (2006); " $\sqrt{ }$ " indicates that this type AGN has been found; "Panda" AGNs are those Seyfert 2 galaxies that have no BLR, but have high accretion rates $(\sim 0.25)$; "?" means that their existence is unconfirmed, and that we suggest searching for them in the SDSS. The blue types of AGNs are predicted by the present paper.

LIL regions (Warner et al. 2003, 2004) lends support to the prediction of our model. Detailed theoretical discussions can be found in W11. Fourth, the present model clearly predicts an intrinsic connection between BLR and NLR through outflows. This section is devoted to discussing these key tests from observations.

\subsection{Observational Appearance During Phase I}

The diversity of AGNs is often attributed mainly to differences in the orientation of a dusty torus with respect to the observer's sight line (Antonucci 1993). Considerable evidence has been found in favor of this scenario, such as the presence of polarized BLRs in some Seyfert 2 galaxies and the larger amount of absorbing material in Seyfert 2 s observed at X-ray wavelengths. However, over the last decade there has been increasing evidence suggesting that this orientation-based unification scheme is not the whole story. Some optically identified type I AGNs have quite large absorption in the X-rays (Fiore et al. 2001; Mateos et al. 2005; Cappi et al. 2006). Some type II AGNs are found without X-ray absorption (Pappa et al. 2001; Panessa \& Bassani 2002; Barcons et al. 2003; Caccianiga et al. 2004; Corral et al. 2005; Wolter et al. 2005; Bianchi et al. 2008; Brightman \& Nandra 2008). These results directly confront the simple version of the dusty torus model, and have motivated suggestions of evolving BLRs and/or varying accretion rates in addition to the orientation-based effects (Panessa \& Bassani 2002; Wang \& Zhang 2007). On the other hand, the complex range of observed properties of intermediate Seyfert galaxies, such as Seyfert 1.8 and Seyfert 1.9 (Trippe et al. 2010), implies that non-Seyfert 1 galaxies are composed of intrinsically different populations. It has been realized that (1) partial obscuration by a clumpy torus may explain the transition from type II to I; (2) low accretion rates can dilute the clouds in the BLRs; and (3) abnormal gas-to-dust ratios in the torus can cause AGN to appear as type II objects at optical wavelengths, but without significant absorption of X-rays. It is plausible that the phase I objects predicted by the present model appear among these abnormal objects.

For convenience, Table 3 lists the known types of AGNs and the likely parameters of their central engines. In the following subsections, we discuss the relationship of these different types of AGNs to the predictions of the present model.

\subsubsection{Panda AGNs: Non-BLR AGNs with Normal Accretion Rates}

It is often assumed that all type II AGNs have hidden BLRs. This idea is based on observations of Seyfert 2 galaxies that in polarized light show broad Balmer lines due to scattered light coming from a BLR obscured by a dusty torus (Antonucci \& Miller 1985; Antonucci 1993). Recently, it has been discovered that some type II AGNs show a deficit of the polarized broad lines (Gu \& Huang 2002; Nicastro et al. 2003), and have accretion rates lower than a critical value of $L_{\text {bol }} / L_{\text {Edd }} \sim 10^{-3}$ (Nicastro et al. 2003; Wang \& Zhang 2007; Elitzur \& Ho 2009). This has been confirmed by further observations (Tran et al. 2011). These objects are called "true" type II AGNs, or unabsorbed non-hidden BLR AGNs. The present model predicts a new type of AGNs, which have normal accretion rates and no BLRs. Since phase I is relatively short, AGNs in this state are rare. We designate those as "Panda" $\mathrm{AGNs}^{7}$ in order to distinguish them from objects with low accretion rates. There is no doubt that the existence of this kind of AGN is a key test of the present model.

Wang \& Zhang (2007) systematically examined a broad sample of published AGN spectra in the light of the unification scheme and the evolutionary influence of SMBH growth. One object of particular interest in their sample is NGC 3660, which shows no polarized broad lines (Tran 2001). Its [O III] luminosity is estimated to $6.5 \times 10^{41} \mathrm{erg} \mathrm{s}^{-1}$ (Kollatschny et al. 1983). With the bolometric luminosity of $L_{\mathrm{Bol}} \sim 10^{45} \mathrm{erg} \mathrm{s}^{-1}$, along with the SMBH mass $M_{\bullet} \sim 10^{7} M_{\odot}$ estimated from the Magorrian relation (Magorrian et al. 1998), we find an Eddington ratio of about unity. NGC 3660 is the only such object in the Wang \& Zhang (2007) sample. Panessa et al. (2009) have more recently discussed an interesting Seyfert 1.8 galaxy, Q2130-431, that has weak broad $\mathrm{H} \alpha$ and $\mathrm{H} \beta$ emission. It has an Eddington ratio of $L_{\text {Bol }} / L_{\text {Edd }} \sim 0.4$, absorption column density $N_{\mathrm{H}}<10^{20} \mathrm{~cm}^{-2}$, and a Balmer decrement of 0.34 in light of $\mathrm{H} \beta / \mathrm{H} \alpha$ ratio, indicating that the BLR is not suffering from heavy reddening and is not obscured. These authors concluded that the weakness of BLR in Q2130-431 is intrinsic in spite of the high accretion rates. This object seems to challenge the popular scenario that the absence of a BLR is driven by the low Eddington ratios.

\footnotetext{
7 Our term "Panda" is meant to convey the idea these are a rare and hard-to-find species, but like their namesakes in the animal kingdom, one that nevertheless does exist.
} 
However, this could be clear evidence for the presence of a transient BLR in phase I suggested by the present model. Though the completeness of the Wang \& Zhang (2007) sample combined with the results of Panessa et al. (2009) is uncertain, the "Panda" AGNs constitute only $\sim 2 / 245=0.8 \%$ of the population, which is consistent with the prediction of the present model for the fraction of AGNs in phase I (see Table 2).

There is increasing evidence for the presence of type II AGNs with low X-ray absorption and high accretion rate, for example, 1ES $1927+654$ with $N_{\mathrm{H}} \sim 7 \times 10^{20} \mathrm{~cm}^{-2}$ and $L_{0.1-2.4 \mathrm{keV}} \sim 5 \times 10^{43} \mathrm{erg} \mathrm{s}^{-1}$ (Boller et al. 2003), and ESO 416-G00211 type II AGNs with $N_{\mathrm{H}}<3 \times 10^{20} \mathrm{~cm}^{-2}$ and $L_{2-10 \mathrm{keV}} \sim 4 \times 10^{43}$ and PMN J0623-4636 with $N_{\mathrm{H}} \sim$ $3 \times 10^{19} \mathrm{~cm}^{-2}$ and $L_{2-10 \mathrm{keV}} \sim 2 \times 10^{44} \mathrm{erg} \mathrm{s}^{-1}$ (Gallo et al. 2006). These typical Seyfert galaxies contain SMBHs with $10^{7} M_{\odot}$, they thus have Eddington ratios between 0.1 and 1 as determined from their X-ray luminosities. Though the reason why type II AGNs have such low absorption remains a matter of debate, they are candidates to be the "Panda" AGNs in the present model. Future polarization observations of these objects should be made to search for obscured BLRs.

\subsubsection{Type II Quasars}

Type II quasars are the more luminous analogues of the type II AGN discussed above, showing only narrow emission lines. They are rare compared to type I quasars, but a considerable number of them have been found in the large Sloan Digital Sky Survey (SDSS) QSO sample (e.g., Zakamska et al. 2003, 2005). Do these include Panda-type quasars? Colors of most of these objects fall between those of galaxies and quasars (Zakamska et al. 2003), implying a nuclear continuum obscured by a dusty torus together with underlying continuum contamination by their host galaxies. Chandra observations of 12 type II quasars find that they generally have high absorbing column density, $N_{\mathrm{H}}>10^{22} \mathrm{~cm}^{-2}$ (Vignali et al. 2006), but this is a very limited sample. According to the model, non-BLR "Panda" quasars should be detected with a probability of $10^{-2}$ from the SDSS.

We made a further search for these Panda-type objects in the SDSS sample from SDSS DR7 (those objects are actually type II quasars in light of Zakamska et al. 2003, 2005), using the criteria of color and line width. We give an example of those objects labeled by phase I as shown in Figure 9. This quasar has a weak [O II] line and hence the continuum is less contaminated by young stars. The [O III] (FWHM) is about $660 \mathrm{~km} \mathrm{~s}^{-1}$ and the dispersion velocity $\sigma=\mathrm{FWHM}([\mathrm{O} \mathrm{III}]) / 2.35 \sim 280 \mathrm{~km} \mathrm{~s}^{-1}$. We have $M_{\bullet} \sim 10^{8.7} M_{\odot}$ from $M_{\bullet}-\sigma$ relation (Tremaine et al. 2002). From the [O III] luminosity of about $10^{43} \mathrm{erg} \mathrm{s}^{-1}$, we find its bolometric luminosity to be $L_{\mathrm{Bol}} \sim 3500 L_{[\mathrm{OIII}]} \approx$ $6 \times 10^{46} \mathrm{erg} \mathrm{s}^{-1}$ and the Eddington ratio to be $L_{\mathrm{Bol}} / L_{\mathrm{Edd}} \sim 0.9$. This object has a normal quasar luminosity as measured from its continuum, implying that its central engine is viewed directly, but that there is no BLR. Our model can explain the absence of the BLR in quasars and AGNs with normal Eddington ratios.

We have to point out that identification of "Panda" quasars should deal with other properties as argued for "Panda" AGNs in Section 5.1.1. In principle, some of them should resemble to weak emission-line quasars (Shemmer et al. 2010). In the future it will be well worth carrying out a systematic search for the Panda-type quasars predicted to exist in the SDSS, and then measuring the properties of those AGNs.

\subsection{Appearance of Evolving BLRs: A Spectral Sequence}

The observed broad emission lines from AGNs and quasars exhibit a wide range of central line shifts, profiles, and EWs (e.g., Hu et al. 2008a, 2008b; Marziani et al. 2010). Here we attempt to build up a spectral sequence of broad lines in light of the BLR states predicted for phases II-III-IV in our model.

Based on the $\mathrm{H} \beta$ profiles, $\mathrm{Hu}$ et al. (2008b) roughly divided a sample of approximately 50,000 SDSS quasars into three classes: (1) objects with redshifted intermediate and broad $\mathrm{H} \beta$ components; $(2)$ ones with non-shifted intermediate and broad $\mathrm{H} \beta$; and (3) ones with only broad components of $\mathrm{H} \beta$. Finally, the maximum $\mathrm{EW}$ of $\mathrm{H} \beta$ in this sample is $\mathrm{EW} \sim 500 \AA$. Figure 9 shows the spectral classes of SDSS quasars. Figure 9(a) shows an example of a possible phase I AGN, as discussed in the previous section. Figures 9 (b) and (c) show the $\mathrm{H} \beta$ lines with redshifted intermediate components which characterize class (1) objects. We interpret these as corresponding to phase II of our model, during which cold clouds return to the SF disk due to the mass circulation between the CAS and the disk. From panel (b) to panel (c), the redshift of the intermediate $\mathrm{H} \beta$ decreases. This corresponds to an increase in the CAS density, leading to a decrease in the infall velocity. When the infall velocity tends to zero, the BLR enters phase III. We associate this phase with the Hu et al. (2008b) class (3) objects, such as those shown in Figures 9(d) and (e). Due to the strong dynamical destruction process, cold clouds are not able to survive long enough to sink into the SF disk. Finally, in Figures $9(f)$ and (g), we show the two objects from $\mathrm{H} 08$ with the maximum $\mathrm{H} \beta$ EW. These two quasars are believed to be in phase IV, i.e., the collapsing state of the BLR evolution.

We can use the relative numbers of objects to test these proposed connections between the classes observed by Hu et al. and the phases predicted by our model. The sample of Hu et al. (2008a, 2008b) shows that the fraction of class (1) objects is $11 \%$, while $89 \%$ of the sample are class (2) and (3) objects. Based on the timescales predicted by our model, we predict that phase II objects should comprise roughly $t_{2} /\left(t_{1}+t_{2}+t_{3}\right) \sim 16 \%$ of all AGNs, while phase III objects should make up about $t_{3} /\left(t_{1}+t_{2}+t_{3}\right) \sim 80 \%$ ( $\mathrm{Hu}$ et al. 2008b). This is quite good agreement between the model and the observations, supporting an interpretation that the empirical classes found by $\mathrm{Hu}$ et al. really do represent the progression through the evolutionary phases predicted by our model.

We note that the Fe II emission lines usually have properties similar to those of the intermediate $\mathrm{H} \beta$ components in the sense that the two have consistent redshifts and FWHM, suggesting they originate from the same regions. However, there is still a fraction of objects which show Fe II profiles that are different from those of the intermediate $\mathrm{H} \beta$ component. Some objects have blueshifted intermediate $\mathrm{H} \beta$, while Fe II shows redshifts. We suggest that this is due to the complicated effects of the optical depths in these different lines (Ferland et al. 2009). The optical-passband Fe II lines may be better indicators of the LIL regions than $\mathrm{H} \beta$ since the former are emitted isotropically while the later is strongly inward beamed at higher optical depths.

In order to further test a transition from phase II to III, we make use of SDSS data to show the dependence of infalling velocity on the CAS density. Our model predicts a steady increase in the density of the CAS until the last phase of collapse, namely dynamical friction of clouds with the Compton gas increases, and the covering factor is increasing with time monotonically. This causes the maximum sinking velocity of 


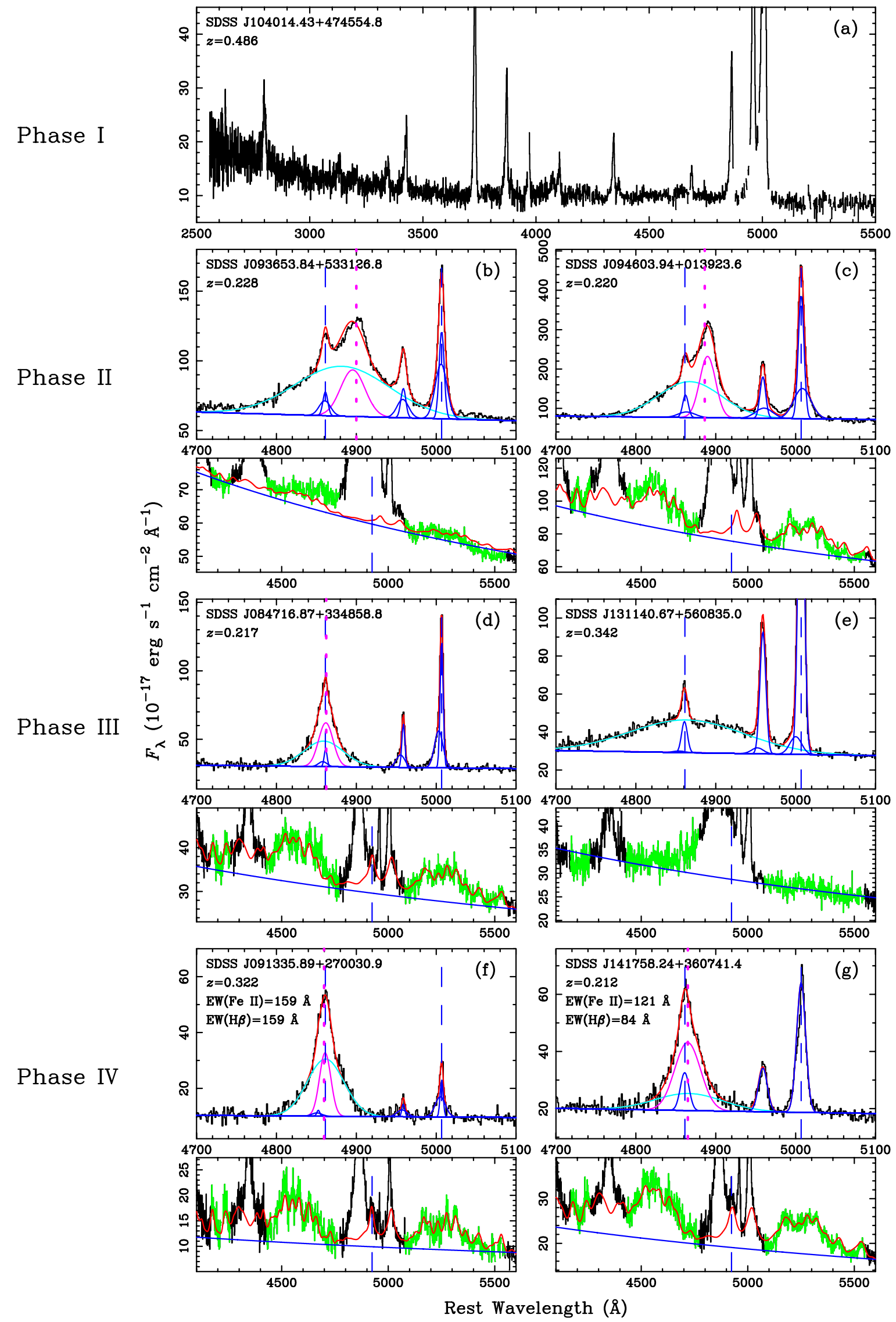

Figure 9. (a) A candidate phase I AGN, from SDSS. The Eddington ratio is about $L_{\mathrm{Bol}} / L_{\mathrm{Edd}} \sim 0.9$. See the text for a detailed explanation. (b)-(g) Spectral sequences of the broad emission-line profiles corresponding to phases II-IV as indicated. A redshifted component of $\mathrm{H} \beta$ appears in phase II and disappears in phase III. Emission lines reach their maximum equivalent width and the BLR is ready to collapse in phase IV. The appearance percentage of each phases can be found in Table 2 , agreeing with the observational statistics from Hu et al. (2008a, 2008b). 

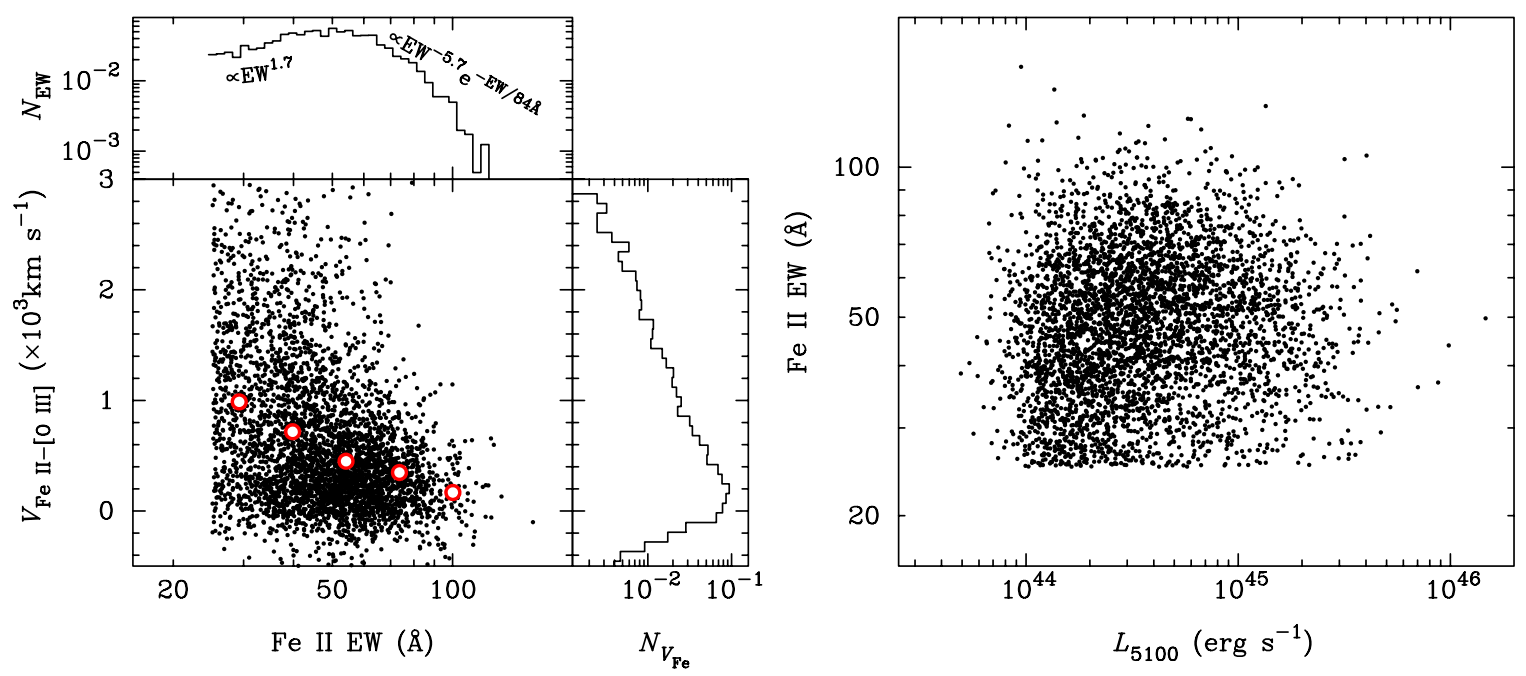

Figure 10. Left panels: the sinking velocity of Fe II clouds vs. the equivalent width. The Fe II equivalent width is obtained by $\mathrm{EW}=F(\mathrm{Fe}$ II $) / F_{5100}$, where $F(\mathrm{Fe}$ II $)$ and $F_{5100}$ are fluxes of Fe II between $4434 \AA$ and $4684 \AA$, and $5100 \AA$, respectively. The density of Compton gas is proportional to the number density of clouds, implying the EW as an indicator of covering factor. We use the EW as an indicator of the CAS density. The data show an anti-correlation of the velocity with the EW, which is consistent with the model. We divided the EW of Fe II lines into five bins. The red circles around white interiors show the averaged value of the infalling velocity of clouds in each bin. Upper-left panel: the distribution of the EW(Fe II) normalized by the total of 4037 objects. The very steep distribution of high EW implies the presence of objects with maximum EW. The $N_{V_{\mathrm{Fe}}}$ panel shows the distribution of Fe II cloud velocity. Right panel: test to determine if there is an anti-correlation between EW (Fe II) and the ionizing luminosity $L_{5100}$. The absence of a correlation implies that the maximum EW of Fe II lines is an intrinsic feature rather than being caused by the flux limit of SDSS survey.

the clouds to decrease as the covering factor increases. Since the Fe II photons from the emitting clouds are more isotropic than $\mathrm{H} \beta$ (Ferland et al. 2009), the EW of the Fe II lines could be a good indicator of the BLR covering factor since the reprocessed emission will be isotropic for Fe II lines (while for $\mathrm{H} \beta$ formed at large column densities it will be anisotropic). We predict an inverse correlation between the EW of Fe II and its redshift (the sinking velocity to the SF disk). Figure 10 tests this prediction, using data from our systematic study of the properties of $\mathrm{H} \beta$ and Fe II lines in SDSS QSOs (Hu et al. 2008a, 2008b). The available data show large scatter, but the averaged points show a clear trend. The sinking velocity does anti-correlate with the covering factor, as predicted.

Finally, we consider the last stage of BLR evolution. The large EWs of $\mathrm{H} \beta$ and $\mathrm{Fe}$ II are the unique observable characteristic of phase IV. For EWs exceeding some critical limit, the entire CAS will be cooled through line cooling. From the Fe II lines, we have the covering factor of the Fe II clouds as $\mathcal{C} \sim 100 \%$. We identify them in the last stage of the BLR. Furthermore, the upper-left panel in Figure 10 shows the observed distribution of the Fe II EWs. We find that it follows $N_{\mathrm{EW}} \propto \mathrm{EW}^{1.7}$ for sources with low EW whereas for high $\mathrm{EW}$ it has the dependence $N_{\text {EW }} \propto \mathrm{EW}^{-5.7} \exp (-\mathrm{EW} / 84 \AA)$. We have a cutoff EW of $\mathrm{EW}_{\text {cutoff }}=84 \AA$, indicating that there is a maximum EW of emission lines, corresponding to phase IV. Sources with $\mathrm{EW}>\mathrm{EW}_{\text {cutoff }} \sim 84 \AA$ will be in phase IV. They constitute about $4 \%$ of the total objects in the $\mathrm{Hu}$ et al. sample. This fraction agrees well with $\sim 1.8 \%$ predicted by the present model (Table 2).

We have tested to see if the maximum EW could be a selection effect due to the flux limit of the SDSS survey. In Figure 10 (right), we plot the potential correlation of EW(Fe II) with the ionizing luminosity. The non-correlation between the two directly shows that the maximum is not caused by the fluxlimit effects. We would like to stress that the clouds are optically thin for Fe II photons, making Fe II EW a robust indicator of the covering factor. The maximum Fe II EW indicates the last stage (phase IV) of the transient BLR.

\subsection{Intrinsic Connection between NLR and BLR: Outflowing Clouds}

The cloud outflows which we predict will be ejected from BLR-I by radiation pressure have the important implications that the clouds may undergo expansion and become highly ionized gas (HIG), which produces many features in X-rays. Although absorption features due to HIG were first clearly seen in AGN with ROSAT (Turner et al. 1993), most of the fundamental physical properties of this component remain a matter for debate. The HIG appears as absorption lines, such as $\mathrm{O}$ VII $(0.74 \mathrm{keV})$ and $\mathrm{O}$ VIII $(0.87 \mathrm{keV})$ in type I AGNs (Reynolds 1997; George et al. 1998; Kaspi et al. 2000) whereas it is seen as emission lines in type II AGNs (Turner et al. 1997; Netzer et al. 1998). The HIG is variously suggested to be intrinsically related with BLR clouds (Reynolds \& Fabian 1995), or to have evaporated off "bloated stars" (Netzer 1996), or to be gas evaporated off the dusty torus obscuring the nucleus (Krolik \& Kriss 1995), or to be a wind driven off the accretion disk (Bottorff et al. 2000). In at least one object, MR 2251-178, the general characteristics of the UV and X-ray absorbers are consistent with them having originated in the same gas (Kaspi et al. 2004). A brief summary is given by Risaliti (2010). The dynamics of the radiatively accelerated HIL clouds predicted by our model is worth investigating to see if these clouds really can be the progenitors of the observed HIG, and it will be very important to determine the metallicity of the HIG through X-ray spectroscopy. Future work on the comparison of warm absorbers with the properties of clouds in the HIL BLR is also well worth carrying out to examine the prediction of the present model through the observational tests of ultraviolet C IV absorption lines with X-ray absorption.

Furthermore, we find a strong correlation between the BLR and NLR metallicity in a sample of quasars observed with Hubble Space Telescope (HST; J.-M. Wang et al. 2011, in preparation). This lends strong support to there being a connection between these two regions, even though they are separated by the large spatial distance of $\sim 1.0 \mathrm{kpc}$. 


\subsection{Other Observational Tests}

Another firm prediction of our model is that the HIL lines (BLR-I) will have higher metallicity than the LIL lines (BLRII). Metallicity in quasars can be estimated by measuring the $\mathrm{N} / \mathrm{C}$ and $\mathrm{N} / \mathrm{O}$ abundance ratio by using the $\mathrm{N} \mathrm{v} / \mathrm{C}$ IV and $\mathrm{N}$ III]/ C III] methods described by Hamann \& Ferland (1999), Hamann et al. (2002) and Baldwin et al. (2003b). As we have discussed in Paper I, the expected differences may have already been found, with $Z_{\mathrm{HIL}} \sim 5 Z_{\mathrm{LIL}}$ from the stacked spectra (Warner et al. 2004). Additional checks of these results are badly needed.

Finally, future reverberation mapping of the AGNs that are thought to represent different phases in our model could provide observational tests of the model (like Denney et al. 2009, 2010). The self-gravitating part of the accretion disk has been regarded as a likely source of the BLR for a quite long time (e.g., Collin \& Hure 2001; Bian \& Zhao 2002). The argument is favored by the fact that the self-gravitation radius is about the same as the reverberation mapping radius. Our results are also generally compatible with the well-known result from reverberation mapping that the HIL lines are formed at smaller radii and the LIL lines at larger radii (Peterson \& Wandel 1999), but a detailed comparison is beyond the scope of this paper. Mont Carlo simulations of the reverberation mapping relation will be carried out in a separate paper.

\section{DISCUSSION}

\subsection{Brief Comparison with Other Models}

It is generally thought that BLRs are composed of a twophase medium (Krolik et al. 1981). Properties of photoionized cold clouds formed through thermal instability can be predicted and generally agree with observations. But key issues have long remained in doubt. These include the origin of the hot-phase gas, the evolution of the hot gas and also of the cold BLR clouds, and the geometry of the BLR. The present model makes clear predictions about the properties of the BLR and clouds, which are testable from observations. We now make a brief comparison of the present model with previously existing ones.

Table 4 lists existing models of the BLRs. ${ }^{8}$ We divided them into five types in light of their main features. They are (1) model type A employs the outer part of the accretion disk, which is irradiated by the central part; (2) model type B deals with outflows which are approximately parallel to the surface of the accretion disk; (3) model type $\mathrm{C}$ invokes clouds bound by magnetic fields which are poloidal perpendicular to the disk; (4) model type D employs gas stirred by stars in a central cluster; (5) model type E uses bloated stars; and (6) model type F deals with SNexp. We briefly compare them with the present model.

First of all, all models of types A-E are neither able to specify how the BLR gas originates, nor connect the metallicity in the BLR with feeding the SMBH. Model A employs the outer part of the accretion disk (e.g., Collin-Souffrin 1987), which is irradiated by the central engine. Clouds may be formed in the outer region of the accretion disk, but the origin of the high metallicity in the BLR remains open. These models could explain the dependence of the BLR on the accretion rates, but the detailed relationship with the accretion disk is highly uncertain. On the other hand, the presence of non-BLR AGNs with normal Eddington ratios (i.e., the Panda AGNs) strongly challenges

\footnotetext{
8 There has been a considerable amount of work on theoretically understanding BLRs. We list here the models that we know of in the published literature, but there may well be others.
}

model A and also the improved suggestions of $\mathrm{Gu} \&$ Huang (2002), Nicastro et al. (2003), and Elitzur \& Ho (2009). The same is true with models of types B and C. Interaction between stars and the accretion disk has been employed by model D in Artymowicz et al. (1993) and Zurek et al. (1994) and leads to production of metals. However, these models do not attempt to explain the establishments of the BLR geometry or the dynamics of clouds. Model E invokes bloated stars, but it is hard to explain a clear trend relating metallicity and luminosity of AGNs. In particular, there is no space for the huge size of the bloated stars (Laor et al. 2006).

Second, properties of clouds in models A-F are only deduced from the necessary conditions for photoionization in light of the cooling and heating function or from observed features. The present model is capable of predicting cloud properties, dynamics of clouds and how they evolve. This is the advantage. In addition, these are stationary models of the BLR clouds, with very short lifetimes against destruction through dynamical friction with their surroundings. The existing models at best assume some motion of clouds around the SMBH in order to predict the line profiles (e.g., Netzer \& Marziani 2010). In contrast to these previous models, the present model predicts the details of the BLR's evolution with time, giving rise to the episodic appearance and reappearance of BLRs during the AGN lifetime. The dynamics of clouds are fully predicted and are different in phases II and III. The dynamical destruction of clouds is explicitly followed and plays a key role in driving the evolution.

Third, in our model the BLR geometry and dynamics are both fully predicted. They are naturally derived from the dynamics of the CAS, the birthplace of the clouds, The spatial distribution of BLR clouds follows the CAS density profile, but also the initial angular momentum of clouds tracks the rotation of the CAS. The CAS properties in turn follow predictably from the properties of the SF disk. In most other models the geometry is not known, so cannot be tested. We have not yet carried out the numerical simulation of the reverberation mapping relation for the present model, but that needs to be done in the future and compared in detail with observations.

Fourth, the present model suggests that the HIL regions could develop outflows through enhanced radiation pressure and predict a potential connection between the BLR and the NLR. As a consequence of the connection, the outflowing clouds could evolve into warm absorbers appearing in soft $\mathrm{X}$-ray bands. We expect an intrinsic relation between HILs and the warmer absorbers. Outflowing clouds are also employed in other models to explain the BLRs. Spherical outflows (Beltrametti 1981) and ascending winds from the accretion disks (Shlosman et al. 1985) have been investigated. Emmering et al. (1992) studied magnetized outflows perpendicular to the disk whereas Murray \& Chiang (1995) and Chiang \& Murray (1996) considered flows parallel to the disk. Though these outflow models can generally generate the profiles of broad emission lines, the geometry is neither certain, nor do they incorporate the stratified structure of the BLRs.

The advantages of the present model can be summarized as follows.

1. The model suggests that BLR formation is a by-product of feeding SMBHs. Without SNexp driving outward transportation of angular momentum, neither accretion onto the SMBH nor BLR formation is able to proceed. This leads to a natural relationship between high metallicity and accretion onto the SMBH. 
Table 4

Summary of BLR Geometry and Dynamics in AGNs

\begin{tabular}{|c|c|c|c|c|}
\hline Classes & References & Dynamics & Geometry & Notes \\
\hline General & $\begin{array}{l}\text { W59 } \\
\text { KMT81 } \\
\text { C88 }\end{array}$ & $\begin{array}{l}\text { Giant potential of gravity } \\
\text { Cloud-intercloud system in thermal balance }\end{array}$ & $\begin{array}{l}\text { Sphere } \\
\text { HIM } \\
\text { Disk (LIL) + sphere (HIL) } \\
\end{array}$ & Seminal paper of the broad $\mathrm{H} \beta$ line \\
\hline Model A & $\begin{array}{l}\text { S85 } \\
\text { BMS83 } \\
\text { SR85 } \\
\text { RNF89 } \\
\text { W96 } \\
\text { CH01 }\end{array}$ & $\begin{array}{l}\text { Clouds developed from RDR region of AD } \\
\text { Compton heated winds from } \mathrm{AD} \\
\text { Compton heated winds from } \mathrm{AD} \\
\text { Clouds bound by magnetic fields } \\
\text { Compton heated winds from } \mathrm{AD} \\
\text { Outer part of accretion disk }\end{array}$ & 西 & 然 \\
\hline Model B & $\begin{array}{l}\text { H75 } \\
\text { S77 } \\
\text { BM75 } \\
\text { C80 } \\
\text { E80 } \\
\text { N80 } \\
\text { B81 } \\
\text { WMY85 } \\
\text { SN88 } \\
\text { R92 } \\
\text { T92 } \\
\text { CR96 } \\
\text { MC97 } \\
\text { N03 } \\
\text { CH11 }\end{array}$ & $\begin{array}{l}\text { Tidal disruption of stars } \\
\text { Disk winds driven by emission of inner disk } \\
\text { Outflowing clouds driven by AD radiation } \\
\text { "Cometary Star" tail } \\
\text { Outflows } \\
\text { Outflows } \\
\text { SMBH potential } \\
\text { Tidal disruption of stars } \\
\text { Evolved SN remnant } \\
\text { Surface of accretion disk } \\
\text { AD-driven outflows } \\
p_{\text {gas }} \sim p_{\text {rad transition regions }} \\
\text { Radiation pressure-driven winds }\end{array}$ & $\begin{array}{l}\text { Outflow } \\
\text { Disk } \\
\text { Sphere } \\
\text { Outflow + rotating disk } \\
\text { Cusp-like } \\
\text { HIL and LIL } \\
\text { Spherical geometry } \\
\text { Quasi-spherical geometry } \\
\text { Winds from stars } \\
\text { Unbound+bound clouds } \\
\qquad \ldots \\
\ldots\end{array}$ & $\begin{array}{l}p_{\text {gas }} \text { and } p_{\text {rad }} \text { are gas and radiation pressures, respectively. } \\
\text { Is partially favored by blueshifted } \mathrm{H} \beta\end{array}$ \\
\hline Model C & EBS92 & Magnetized winds from disk & & \\
\hline Model D & $\begin{array}{l}\text { ALW93 } \\
\text { VC02 } \\
\text { ZSC94 }\end{array}$ & $\begin{array}{l}\text { Stars interact with accretion disk } \\
\text { Star-disk and star-star interaction } \\
\text { Star-disk interaction }\end{array}$ & $\begin{array}{l}\text { Inflow } \\
\text { Star tail above accretion disk }\end{array}$ & \\
\hline Model E & $\begin{array}{l}\text { AN94/97 } \\
\text { P88 } \\
\text { T89 } \\
\text { K89 } \\
\text { TP02 }\end{array}$ & $\begin{array}{l}\text { Bloated stars interaction } \\
\text { SMBH potential } \\
\text { SMBH potential } \\
\text { SMBH potential } \\
\text { Stellar atmosphere }\end{array}$ & $\begin{array}{l}\text { Sphere } \\
\text { Irradiated stellar chromospheres } \\
\text { Bloated stars } \\
\text { Bloated stars } \\
\qquad \ldots \\
\end{array}$ & NGC 4395 does apply \\
\hline Model F & $\begin{array}{l}\text { P01 } \\
\text { PD85 } \\
\text { This paper }\end{array}$ & $\begin{array}{l}\text { Supernova-QSO wind interactions } \\
\text { Supersonic gas flow interact with obstacles } \\
\text { Clouds bound by SMBHs }\end{array}$ & BLR gas from the SF disk & $\begin{array}{l}\text { Obstacles: SNexp shell and strong stellar winds } \\
\text { Episodic appearance of BLR in four phases }\end{array}$ \\
\hline
\end{tabular}

Notes. All the models involve photoionization as main radiation mechanism. AD: accretion disk; HIL: high-ionization lines; LIL: low-ionization lines; PI: photoionization; RDR: radiation-dominated region; HIM: hot intercloud medium.

References. (AN94/97) Alexander \& Netzer 1994, 1997; (ALW93) Artymowicz et al. 1993; (B81) Beltrametti 1981; (BM75) Blumenthal \& Mathews 1975; (BMS83) Begelman et al. 1983; (C80) Capriotti et al. 1980; (C88) Collin-Souffrin et al. 1988; (CH01) Collin \& Hure 2001; (CH11) Czerny \& Hryniewicz 2011; (CR96) Cassidy \& Raine 1996; (E80) Edwards 1980; (EBS92) Emmering et al. 1992; (K89) Kazanas 1989; (H75) Hills 1975; (KMT81) Krolik et al. 1981; (MC97) Murray \& Chiang 1997; (N80) Netzer 1980; (N03) Nicastro et al. 2003; (P88) Penston 1988; (PD85) Perry \& Dyson 1985; (P01) Pittard et al. 2001; (RNF89) Rees et al. 1989; (R92) Roos 1992; (S77) Shields 1977; (S85) Shlosman et al. 1985; (SN88) Scoville \& Norman 1988; (SR85) Smith \& Raine 1985; (T92) Terlevich et al. 1992; (TP02) Torricelli-Ciamponi \& Pietrini 2002; (T89) Tout et al. 1989; (VC02) Vilkoviskij \& Czerny 2002; (W59) Woltjer 1959; (WMY85) Wandel et al. 1985; (W96) Woods et al. 1996; (ZSC94) Zurek et al. 1994.

2. Cold clouds are produced in an evolving Compton gas originated from the SF disk, avoiding the crisis of the dynamical destruction of clouds, which has not been solved in the classical model. On the contrary, the episodic appearance of the BLR employs the dynamical destruction of clouds, which is a necessary element in the present model.

3. The geometry and dynamics of clouds follow the Compton gas. The BLR is naturally divided into the low- and highionization regions related with different phenomena of emission lines. Outflows from the HIL regions bridge the BLR and NLRs separated by five orders in size scale.

4. The model predicts a new kind of AGNs, which have higher Eddington ratio, but do not have BLRs. This enriches the classes of known AGNs.
5. The model predicts a metallicity gradient between HIL and LIL regions. This is one of the main characteristics of the present model.

The present model has further implications to observations beyond the scope of BLRs as a by-product of feeding the SMBHs. The fact that the BLRs emit more energy than they received from UV and X-rays, which is known as "energy budget problem" (Collin-Souffrin 1986; Netzer 1990) could be explained by supplementing the UV emission with light from the massive stars in the SF disk provided the star formation rates are high enough (see Section 6.2). If this is true, quasars with the energy budget problem (Netzer 1985) should have higher star formation rates, and hence higher metallicity. This possibility should be explored. 
In this paper, we show that BLRs are formed as a consequence of the accretion flow onto the SMBHs. Since the evolving BLRs are not stationary during the AGN lifetime, the BLR will be renewed in the next episode with a duty cycle of a few $10^{3} \mathrm{yr}$. The SMBH activities are composed of (1) BLR episodes and (2) AGN episodes. This indicates that the BLRs will have similar properties in low- and high-redshift quasars. There is no measurable cosmological evolution of SED (Brandt 2007) or of metallicity (Hamann \& Ferland 1993; Warner et al. 2004; Shemmer et al. 2004; Matsuoka et al. 2011). Also as relics of SMBH activities, stellar rings/belts and eventually nuclear compact star clusters (Wang et al. 2010) should be produced during random accretion onto SMBHs (King \& Pringle 2007) as evidenced by the results from $\eta$-equation of SMBH spins (Wang et al. 2009). A future paper will provide a global explanation of fueling SMBH, BLRs, metallicity, nuclear compact star cluster, and evolution of SMBH as they spin down.

\subsection{Future Work}

First of all, we approximate the Compton gas as barotropic gas in a quasi-steady state for discussion of the thermal instability of the Compton gas. This approximation holds provided the supplying timescale is longer than the thermal instability timescale. The present model should be improved for some special cases with fast injection from the SF disk. We simply discuss the fate of the Compton gas with several critical densities. We neglect the detailed contraction of clouds. Second, we do not discuss the detailed dynamics of clouds after their formation from the Compton gas. For simplicity, we simplify their motion to be a spiral path down to the SF disk with the initial angular momentum of their birth. This is important for profiles from the bound clouds in BLRs. Third, the dynamics of outflowing clouds is not discussed with regard to the connection between the BLR and NLR. We plan to investigate the evolution of the outflowing clouds in a self-consistent way, including expansion, involving the swept medium, and metallicity mixture, for the connection between the BLR and the NLR. These processes should produce multiwavelength features. Warm absorbers in X-rays should somehow be linked with the clouds in BLR-I. This could be examined by comparing the properties of UV C IV profiles with the soft X-ray spectrum.

One may ask about two other potential energy sources for heating the CAS. The first is self-heating when the CAS is accreted, through viscosity resembling the $\alpha$-prescription. This is similar to the case studied by Meyer \& Meyer-Hofmeister (1994). The energy flux due to the viscosity is given by $F_{\mathrm{CAS}}=3 G M_{\bullet} \dot{M}_{\mathrm{CAS}} / 4 \pi R^{3}$, where $\dot{M}_{\mathrm{CAS}}$ is the accretion rates of the CAS onto the SMBH. The energy flux from the central engine reads $F_{\mathrm{AD}}=\eta \dot{M}_{\bullet} c^{2} / 4 \pi R^{2}$. We have $F_{\mathrm{CAS}} / F_{\mathrm{AD}}=$ $1.5 \times 10^{-3} r_{3}^{-1}\left(\dot{M}_{\mathrm{CAS}} / \dot{M}_{\bullet}\right) \ll 1$. However, the leakage of the CAS gas through accretion would play an important role in the evolution of the CAS. Actually, the accretion rate in the CAS is much less than the injection rate from the SF disk. This guarantees the results presented in this paper. The second possible additional energy source is radiation from the SF disk. The energy flux from the SF disk is $F_{\mathrm{SF}}=\eta_{*} \dot{\Sigma}_{*} c^{2}$, where $\eta \sim 10^{-3}$ for a Salpeter initial mass function (Thompson et al. 2005) and $\dot{\Sigma}_{*}$ is the surface density of the star formation rate. We have $F_{\mathrm{SF}} / F_{\mathrm{AD}}=\left(\eta_{*} \eta^{-1}\right)\left(\dot{\mathcal{R}}_{*} / \dot{M}_{\bullet}\right) \sim 0.1$ if $\dot{\mathcal{R}}_{*} \sim 10 \dot{M}_{\bullet}$ and $\eta=0.1$. So this energy supply to the CAS could be important in some cases. Especially, its contribution may change the
Compton temperature, but this depends on its SED (e.g., Sirko $\&$ Goodman 2003). Future work should include the leakage of the CAS mass through accretion onto the SMBH and the heating of the SF disk itself.

Accurate cooling functions should be used to determine the new thermal equilibrium to obtain the final column density and then the dynamics of clouds in the radiation fields. Furthermore, we may, in principle, include other heating mechanism discussed in Krolik et al. (1981). The dynamics of the clouds should be specified more completely by including the friction due to the CAS, radiation pressure, and even evaporation of clouds and condensations. This will allow us to study the detailed processes of the evolution of clouds.

Composite spectra from the assembly clouds in the BLRs should be created by adding all the spectra from every individual clouds (Ferland \& Baldwin 1999). Line profiles from such a complicated model will be calculated in light of the cloud's dynamics. Clouds in LIL regions only have one orbital lifetime in phase II, and an even shorter lifetime in phase III. This complicates the calculations of the line profiles, as compared to the scheme used by Netzer \& Marziani (2010). Reverberation mapping of broad emission-line regions given in this paper should be done in future. We will carry out these results in a separate paper.

The present model will also need modifications to be able to properly describe those LLAGNs which lack BLRs. For a very low accretion rate, the SF disk will be so thin that the SNexp will break out and develop strong expansion outside of the disk. The central engines of these low-luminosity objects are expected to develop ADAFs with winds that will collide with the supernova winds, producing significant $\gamma$-ray emission. Such objects should manifest themselves as LINERs. The predicted $\gamma$-rays may be observable with Fermi or HESS. A detailed model for this scenario will be developed separately.

\section{CONCLUSIONS}

We have developed a model that describes the way in which an SF region in the outer parts of an AGN accretion disk (beyond the self-gravitation radius) will quite naturally, just as an inevitable result of the accretion process, lead to a two-component broad emission-line region. We have shown that the BLR should appear, disappear, and then reappear in a continuing series of episodes. Within each episode, the BLR will progress through an evolutionary cycle consisting of four phases. This will lead to an observable sequence of quasar broad emission-line spectral types, with predictable characteristics. We have briefly tested these predictions against observations. This episodic model for the BLR predicts: (1) the observed result that $16 \%$ of SDSS quasars show systematic redshifts correlated with the Eddington ratios whereas about $80 \%$ quasars show overlapping profiles of intermediate and broad components; (2) a new class of bright type II AGNs without broad emission lines and with high Eddington ratios; (3) an intrinsic connection of BLR and NLR through the outflows developed from the high-ionization regions; and (4) a significant difference of metallicity between the high- and low-ionization regions. We have described mostly published observational evidence suggesting that each of these predictions is correct.

The BLRs are naturally divided into high- and low-ionization (HIL and LIL) regions. The HIL region is characterized by outflows. The LIL region has a mass circulation with the SF disk and, later, a mass cycle with the CAS in phases II and III, respectively. Properties of clouds are self-consistently obtained 
from the model. An important advantage of the present model is that it employs clouds which are not in stationary states, avoiding the difficulties encountered by models in which dynamical destruction of the clouds must be prevented.

The striking predictions of our model have been compared to SDSS data in a preliminary way. Further observational tests should in the future provide a more detailed test of this transient BLR model.

We appreciate the stimulating discussions among the members of IHEP AGN group. J.M.W. is grateful to H.-Y. Zhou for interesting conversations about the non-BLR quasars. Y.$\mathrm{R}$. Li is thanked for plotting the cartoon of broad-line regions. The research is supported by NSFC-10733010 and -10821061, and 973 project (2009CB824800). J.A.B. and G.J.F. are grateful to J.-M. Wang and the Institute of High Energy Physics of the Chinese Academy of Sciences for their hospitality. J.A.B. acknowledges support by NASA grant NNX10AD05G and G.J.F. by NSF (0908877, 1108928, and 1109061), NASA (07ATFP07-0124, 10-ATP10-0053, and 10-ADAP10-0073), JPL (RSA No. 1430426), and STSci (HST-AR-12125.01 and HSTGO-12309).

\section{APPENDIX A}

\section{AN ISOTHERMAL COMPTON ATMOSPHERE}

As we shown in Figure 3, the CAS is not an isothermal atmosphere. However, it could be a good approximation, providing very simple results. In this Appendix, we discuss the isothermal CAS. For $\gamma=1$, Equations (40) and (41) yield solution as

$$
K_{0} \ln \rho_{0}+\Phi=\int \Omega^{2} R d R
$$

where $K_{0}=k T_{\mathrm{Comp}} / m_{p}$ and Equation (46) can be re-cast as

$$
\rho_{0}(r, h)=\exp \left\{\frac{m_{p} c^{2}}{k T_{\text {Comp }}}\left[\frac{\omega_{0}^{2} r_{\text {out }}^{2 q-3}}{4(1-q)} \frac{1}{r^{2(q-1)}}+\frac{1}{2\left(r^{2}+h^{2}\right)^{1 / 2}}+C_{0}\right]\right\} .
$$

The results are numerically similar to that as shown in Figures 6 and 7 and we thus omit to carry them out. It should be noted that the index $\gamma \neq 1$ in the development of thermal instability for an isothermal CAS.

\section{APPENDIX B}

\section{DISPERSION RELATION}

The thermal instability of a rotating corona above a galactic disk has been studied by Binney et al. (2009) and Nipoti (2010), however the results of his analysis cannot directly be scaled down to the BLR case. Following Nipoti (2010), the perturbation equations (48)-(52) give

$$
\left[\begin{array}{ccccc}
i k_{R} \rho_{0} & i k_{z} \rho_{0} & 0 & -i \hat{\omega} & 0 \\
-i \hat{\omega} \rho_{0} & 0 & -2 \Omega \rho_{0} & -\mathcal{A}_{p R} c_{0}^{2} & i k_{R} \\
0 & -i \hat{\omega} \rho_{0} & 0 & -\mathcal{A}_{p z} c_{0}^{2} & i k_{z} \\
\Omega+\Omega_{R} & \Omega_{z} & -i \hat{\omega} & 0 & 0 \\
\mathcal{A}_{p R}-\gamma \mathcal{A}_{\rho R} & \mathcal{A}_{p z}-\gamma \mathcal{A}_{\rho z} & 0 & \left(i \hat{\omega}-\omega_{d}\right) \gamma \rho_{0}^{-1} & -i \hat{\omega} p_{0}^{-1}
\end{array}\right]\left[\begin{array}{c}
v_{R}^{1} \\
v_{z}^{1} \\
v_{\phi}^{1} \\
\rho_{1} \\
p_{1}
\end{array}\right]=\left[\begin{array}{c}
0 \\
0 \\
0 \\
0 \\
0
\end{array}\right],
$$

where $\omega_{\mathrm{d}}=\omega_{\mathrm{c}}+\omega_{\mathrm{th}}, \Omega_{z}=\partial(R \Omega) / \partial z$, and $\Omega_{R}=\partial(R \Omega) / \partial R$. Here, $\mathcal{A}_{p R}=\left(\partial p_{0} / \partial R\right) / p_{0}$ and $\mathcal{A}_{p z}=\left(\partial p_{0} / \partial z\right) / p_{0}$ are the inverse of the pressure scale length and scale height, respectively; $\mathcal{A}_{\rho R}=\left(\partial \rho_{0} / \partial R\right) / \rho_{0}$ and $\mathcal{A}_{\rho z}=\left(\partial \rho_{0} / \partial z\right) / \rho_{0}$ are the inverse of the density scale length and scale height, respectively. The non-zero solution of the above equations gives

$$
\left|\begin{array}{ccccc}
i k_{R} \rho_{0} & i k_{z} \rho_{0} & 0 & -i \hat{\omega} & 0 \\
-i \hat{\omega} \rho_{0} & 0 & -2 \Omega \rho_{0} & -\mathcal{A}_{p R} c_{0}^{2} & i k_{R} \\
0 & -i \hat{\omega} \rho_{0} & 0 & -\mathcal{A}_{p z} c_{0}^{2} & i k_{z} \\
\Omega+\Omega_{R} & \Omega_{z} & -i \hat{\omega} & 0 & 0 \\
\mathcal{A}_{p R}-\gamma \mathcal{A}_{\rho R} & \mathcal{A}_{p z}-\gamma \mathcal{A}_{\rho z} & 0 & \left(i \hat{\omega}-\omega_{d}\right) \gamma \rho_{0}^{-1} & -i \hat{\omega} p_{0}^{-1}
\end{array}\right|=0
$$

yielding

where $\hat{n}=-i \hat{\omega}$, the coefficients are

$$
a_{5} \hat{n}^{5}+a_{3} \hat{n}^{3}+a_{2} \hat{n}^{2}+a_{1} \hat{n}+a_{0}=0
$$

$$
\begin{gathered}
a_{5}=-\frac{\rho_{0}}{p_{0}} \\
a_{3}=-\gamma\left(k_{R}^{2}+k_{z}^{2}\right)-i \gamma\left(k_{R} \mathcal{A}_{\rho R}+k_{z} \mathcal{A}_{\rho z}\right)-\frac{2 \rho_{0} \Omega}{p_{0}}\left(\Omega+\Omega_{R}\right),
\end{gathered}
$$




$$
\begin{aligned}
& a_{2}=-\gamma\left(k_{R}^{2}+k_{z}^{2}\right) \omega_{\mathrm{d}}=-\gamma k^{2} \omega_{\mathrm{d}}, \\
& a_{1}=a_{11}+a_{12}+a_{13}+\left(a_{14}+a_{15}\right) i,
\end{aligned}
$$

and

$$
a_{0}=2 \gamma k_{z} \Omega \omega_{d}\left[k_{R} \Omega_{z}-k_{z}\left(\Omega+\Omega_{R}\right)\right],
$$

where

$$
\begin{aligned}
& a_{11}=c_{0}^{2}\left(k_{R} \mathcal{A}_{p z}-k_{z} \mathcal{A}_{p R}\right)^{2} \sim c_{0}^{2}\left(k R^{-1}\right)^{2}, \\
& a_{12}=c_{0}^{2} \gamma\left(k_{z} \mathcal{A}_{\rho R}-k_{R} \mathcal{A}_{\rho z}\right)\left(k_{R} \mathcal{A}_{p z}-k_{z} \mathcal{A}_{p R}\right) \sim c_{0}^{2} \gamma\left(k R^{-1}\right)^{2}, \\
& a_{13}=2 \gamma k_{z} \Omega\left[k_{R} \Omega_{z}-k_{z}\left(\Omega+\Omega_{R}\right)\right] \sim 2 \gamma k^{2} \Omega^{2}, \\
& a_{14}=-2 \gamma \Omega k_{z}\left[\mathcal{A}_{\rho z}\left(\Omega+\Omega_{R}\right)-\mathcal{A}_{\rho R} \Omega_{z}\right] \sim 2 \gamma \Omega^{2}\left(k R^{-1}\right), \\
& a_{15}=2 \Omega \Omega_{z}\left(k_{R} \mathcal{A}_{p z}-k_{z} \mathcal{A}_{p R}\right) \sim 2 \Omega^{2}\left(k R^{-1}\right) .
\end{aligned}
$$

For low-frequency perturbations, we can use the approximations

$$
\omega^{2} \ll c_{0}^{2}\left(k_{R}^{2}, k_{z}^{2}\right) ; \text { and }\left(\Omega^{2}, \Omega_{z}^{2}, \Omega_{R}^{2}\right) \ll c_{0}^{2}\left(k_{z}^{2}, k_{R}^{2}\right),
$$

whereas for the short-wavelength case we have the approximations

$$
\left(\left|k_{R}\right|,\left|k_{z}\right|\right) \gg\left(\left|\mathcal{A}_{\rho R}\right|,\left|\mathcal{A}_{p z}\right|,\left|\mathcal{A}_{p z}\right|,\left|\mathcal{A}_{p R}\right|\right) .
$$

The fifth-order term vanishes in the dispersion relation.

The terms of $a_{3}$ are of order $\left|a_{31}\right|=\gamma\left(k_{R}^{2}+k_{z}^{2}\right) \sim \gamma k^{2},\left|a_{32}\right|=\gamma\left(k_{R} \mathcal{A}_{\rho R}+k_{z} \mathcal{A}_{\rho z}\right) \sim \gamma k R^{-1}$, and $\left|a_{33}\right|=2 \rho_{0} \Omega\left(\Omega+\Omega_{R}\right) / p_{0} \sim \Omega^{2} / c_{0}^{2}$. We find that $\left|a_{31} / a_{32}\right| \sim k R \gg 1$ and $\left|a_{31} / a_{33}\right| \sim \gamma k^{2} c_{0}^{2} / 2 \Omega^{2} \gg 1$. We then have the simplified form

$$
a_{3}=-\gamma\left(k_{R}^{2}+k_{z}^{2}\right)=-\gamma k^{2} .
$$

The coefficients of $a_{1 i}$ are of $a_{11} \sim a_{12} \sim a_{13}$, and $a_{13} \gg\left(a_{14}, a_{15}\right)$, we have

$$
a_{1}=a_{11}+a_{12}+a_{13} \text {. }
$$

The coefficients can be simplified through the operator introduced by Balbus (1995), which is defined by

$$
\mathcal{D}=\frac{k_{R}}{k_{z}} \frac{\partial}{\partial z}-\frac{\partial}{\partial R}
$$

We use the following relations for convenience:

$$
k_{R} \mathcal{A}_{p z}-k_{z} \mathcal{A}_{p R}=k_{z} \mathcal{D} \ln p_{0} ; k_{R} \mathcal{A}_{\rho z}-k_{z} \mathcal{A}_{\rho R}=k_{z} \mathcal{D} \ln \rho_{0}
$$

and

$$
\mathcal{D} s_{0}=\mathcal{D} \ln p_{0}-\gamma \mathcal{D} \ln \rho_{0},
$$

where the entropy $s_{0}=\ln p_{0} \rho_{0}^{-\gamma}$. We have other relations

$$
k_{R} \Omega_{z}-k_{z} \Omega_{R}=k_{z} \mathcal{D}(R \Omega)
$$

and

$$
\mathcal{D}(R \Omega)-\Omega=\frac{\mathcal{D}\left(R^{4} \Omega^{2}\right)}{2 R^{3} \Omega} .
$$

With the help of Equations (B14)-(B17), we have

$$
\begin{gathered}
a_{11}=c_{0}^{2} k_{z}^{2}\left(\mathcal{D} \ln p_{0}\right)^{2}, \\
a_{12}=-c_{0}^{2} \gamma k_{z}^{2}\left(\mathcal{D} \ln p_{0}\right)\left(\mathcal{D} \ln \rho_{0}\right), \\
a_{13}=\frac{\gamma k_{z}^{2}}{R^{3}} \mathcal{D}\left(R^{4} \Omega^{2}\right),
\end{gathered}
$$




$$
a_{0}=\omega_{\mathrm{d}} \frac{\gamma k_{z}^{2}}{R^{3}} \mathcal{D}\left(R^{4} \Omega^{2}\right)
$$

and

$$
a_{1}=c_{0}^{2} k_{z}^{2}\left(\mathcal{D} \ln p_{0}\right)\left(\mathcal{D} s_{0}\right)+\frac{\gamma k_{z}^{2}}{R^{3}} \mathcal{D}\left(R^{4} \Omega^{2}\right)
$$

We have the dispersion relation as

$$
-\gamma k^{2} \hat{n}^{3}-\gamma k^{2} \omega_{\mathrm{d}} \hat{n}^{2}+\left[c_{0}^{2} k_{z}^{2}\left(\mathcal{D} \ln p_{0}\right)\left(\mathcal{D} s_{0}\right)+\frac{\gamma k_{z}^{2}}{R^{3}} \mathcal{D}\left(R^{4} \Omega^{2}\right)\right] \hat{n}+\omega_{\mathrm{d}} \frac{\gamma k_{z}^{2}}{R^{3}} \mathcal{D}\left(R^{4} \Omega^{2}\right)=0,
$$

reducing to

$$
\hat{n}^{3}+\omega_{\mathrm{d}} \hat{n}^{2}-\left[c_{0}^{2} \frac{k_{z}^{2}}{\gamma k^{2}}\left(\mathcal{D} \ln p_{0}\right)\left(\mathcal{D} s_{0}\right)+\frac{k_{z}^{2}}{k^{2} R^{3}} \mathcal{D}\left(R^{4} \Omega^{2}\right)\right] \hat{n}-\omega_{\mathrm{d}} \frac{k_{z}^{2}}{k^{2} R^{3}} \mathcal{D}\left(R^{4} \Omega^{2}\right)=0 .
$$

Introducing the Brunt-Väisälä frequency

$$
\omega_{\mathrm{BV}}^{2}=-c_{0}^{2} \frac{k_{z}^{2}}{\gamma k^{2}}\left(\mathcal{D} \ln p_{0}\right)\left(\mathcal{D} s_{0}\right)=-\frac{k_{z}^{2}}{k^{2}} \frac{\mathcal{D} p_{0}}{\gamma \rho_{0}} \mathcal{D} s_{0}
$$

and the differential rotation term

$$
\omega_{\text {rot }}^{2}=-\frac{k_{z}^{2}}{k^{2} R^{3}} \mathcal{D}\left(R^{4} \Omega^{2}\right)=-\frac{k_{z}^{2}}{k^{2} R^{3}} \mathcal{D} \ell^{2},
$$

where $\ell=\Omega R^{2}$ is the specific angular momentum, we have the dispersion equation as

$$
\hat{n}^{3}+\omega_{\mathrm{d}} \hat{n}^{2}+\left(\omega_{\mathrm{BV}}^{2}+\omega_{\mathrm{rot}}^{2}\right) \hat{n}+\omega_{\mathrm{rot}}^{2} \omega_{\mathrm{d}}=0 .
$$

The simplified version of Equation (B22) for barotropic gas reads

$$
\omega_{\mathrm{BV}}^{2}=\frac{k_{z}^{2}}{k^{2}} \frac{c_{0}^{2} \mathcal{A}_{p z}^{2}}{\gamma}\left(\frac{\gamma}{\gamma^{\prime}}-1\right)\left(\frac{k_{R}}{k_{z}}-\frac{\mathcal{A}_{p R}}{\mathcal{A}_{p z}}\right)^{2},
$$

where we use the assumption that the pressure is only function of density, and we define

$$
\gamma^{\prime} \equiv \frac{d \ln p_{0}}{d \ln \rho_{0}}
$$

which can be regarded as a local polytropic index. For the overall barotropic gas, $\gamma^{\prime}=\gamma$, we have $\omega_{\mathrm{BV}}^{2}=0$, and the dispersion equation reads

$$
\left(\hat{n}^{2}+\omega_{\mathrm{rot}}^{2}\right)\left(\hat{n}+\omega_{\mathrm{d}}\right)=0
$$

with the solution

$$
\hat{n}^{2}=-\omega_{\text {rot }}^{2} \text { or } \hat{n}=-\left(\omega_{\mathrm{c}}+\omega_{\mathrm{th}}\right)
$$

\section{REFERENCES}

Abbott, D. C. 1982, ApJ, 259, 282

Alexander, T., \& Netzer, H. 1994, MNRAS, 270, 781

Alexander, T., \& Netzer, H. 1997, MNRAS, 284, 967

Antonucci, R. 1993, ARA\&A, 31, 473

Antonucci, R., \& Miller, J. 1985, ApJ, 297, 621

Arav, N., Barlow, T. A., Laor, A., \& Blandford, R. D. 1997, MNRAS, 288, 1015

Artymowicz, P., Lin, D. N. C., \& Wampler, E. J. 1993, ApJ, 409, 592

Balbus, S. 1995, ApJ, 453, 380

Baldwin, J. A., Ferland, G. J., Korista, K. T., Hamann, F., \& Dietrich, M. 2003a, ApJ, 582, 590

Baldwin, J. A., Hamann, F., Korista, K. T., et al. 2003b, ApJ, 583, 649

Barcons, X., Carrera, F. J., \& Ceballos, M. T. 2003, MNRAS, 339, 757

Begelman, M. C., McKee, C. F., \& Shields, G. A. 1983, ApJ, 271, 70

Beltrametti, M. 1981, ApJ, 250, 18

Bian, W., \& Zhao, Y. 2002, A\&A, 395, 465

Bianchi, E., Corral, A., Panessa, F., et al. 2008, MNRAS, 385, 195

Binney, J., Nipoti, C., \& Fraternali, F. 2009, MNRAS, 397, 1804
Blumenthal, G. R., \& Mathews, W. G. 1975, ApJ, 198, 517

Böhringer, H., \& Hensler, G. 1989, A\&A, 215, 147

Boller, T., Voges, W., Dennefeld, M., et al. 2003, A\&A, 397, 557

Boroson, T. 2005, AJ, 130, 381

Boroson, T., \& Green, R. 1992, ApJS, 80, 109

Bottorff, M., Ferland, G., Baldwin, J., \& Korista, K. 2000, ApJ, 542, 644

Brandt, W. N. 2007, in ASP Conf. Ser. 380, At the Edge of the X-ray Universe: Results from the Deepest Extragalactic Surveys, ed. J. Afonso,

H. C. Ferguson, B. Mobasher, \& R. Norris (San Francisco, CA: ASP), 103

Brightman, M., \& Nandra, K. 2008, MNRAS, 390, 1241

Caccianiga, A., Severgnini, P., Braito, V., et al. 2004, A\&A, 416, 901

Cappi, M., Panessa, F., Bassani, L., et al. 2006, A\&A, 446, 459

Capriotti, E., Foltz, C., \& Byard, P. 1980, ApJ, 241, 903

Cassidy, I., \& Raine, D. J. 1996, A\&A, 310, 49

Chiang, J., \& Murray, N. 1996, ApJ, 466, 704

Collin-Souffrin, S. 1986, A\&A, 166, 115

Collin-Souffrin, S. 1987, A\&A, 179, 60

Collin-Souffrin, S., Dyson, J. E., McDowell, J. C., \& Perry, J. J. 1988, MNRAS, 232, 539

Collin, S., \& Hure, J.-M. 2001, A\&A, 372, 50 
Collin, S., Kawaguchi, T., Peterson, B. M., \& Vestergaard, M. 2006, A\&A, 465 75

Collin, S., \& Zahn, J.-P. 2008, A\&A, 477, 419

Corral, A., Barcons, X., Carrera, F. J., Ceballos, M. T., \& Mateos, S. 2005, A\&A, 431, 97

Czerny, B., \& Hryniewicz, K. 2011, A\&A, 525, L8

Denney, K. D., Peterson, B. M., Pogge, R. W., et al. 2009, ApJ, 704, L80

Denney, K. D., Peterson, B. M., Pogge, R. W., et al. 2010, ApJ, 721, 715

Edwards, D. A. 1980, MNRAS, 190, 757

Elitzur, M., \& Ho, L. C. 2009, ApJ, 701, L91

Emmering, R. T., Blandford, R. D., \& Shlosman, I. 1992, ApJ, 385, 460

Ferland, G. 2004, in ASP Conf. Ser. 311, AGN Physics with the Sloan Digital Sky Survey, ed. G. T. Richards \& P. B. Hall (San Francisco, CA: ASP), 161

Ferland, G., \& Baldwin, J. 1999, in ASP Conf. Ser. 162, Quasars and Cosmology, ed. G. Ferland \& J. Baldwin (San Francisco, CA: ASP), 147

Ferland, G., Hu, C., Wang, J.-M., et al. 2009, ApJ, 707, 82

Field, G. B. 1965, ApJ, 142, 531

Fiore, F., Pellegrini, S., Matt, G., et al. 2001, ApJ, 556, 150

Gallo, L. C., Lehmann, I., Pietsch, W., et al. 2006, MNRAS, 365, 688

George, I. M., Turner, T. J., Netzer, H., et al. 1998, ApJS, 114, 73

Gnat, O., \& Sternberg, A. 2007, ApJS, 168, 213

Goodman, J. 2003, MNRAS, 339, 937

Grupe, D., Komossa, S., Leighly, K. M., \& Page, K. L. 2010, ApJS, 187, 64

Gu, Q.-S., \& Huang, J.-H. 2002, ApJ, 579, 205

Hamann, F. W., \& Ferland, G. J. 1992, ApJ, 391, 53

Hamann, F. W., \& Ferland, G. J. 1993, ApJ, 418, 11

Hamann, F. W., \& Ferland, G. J. 1999, ARA\&A, 37, 487

Hamann, F., Korista, K. T., Ferland, G. J., et al. 2002, ApJ, 564, 592

Hills, J. G. 1975, Nature, 254, 295

Ho, L. C. 2008, ARA\&A, 46, 475

Hu, C., Wang, J.-M., Ho, L. C., et al. 2008a, ApJ, 683, L115

Hu, C., Wang, J.-M., Ho, L. C., et al. 2008b, ApJ, 687, 78

Iwasaki, K., \& Tsuribe, T. 2008, MNRAS, 387, 1554

Iwasaki, K., \& Tsuribe, T. 2009, A\&A, 508, 725

Kaspi, S., Maoz, D., Netzer, H., et al. 2005, ApJ, 629, 61

Kaspi, S., Netzer, H., Chelouche, D., et al. 2004, ApJ, 611, 68

Kaspi, S., Smith, P. S., Netzer, H., et al. 2000, ApJ, 533, 631

Kazanas, D. 1989, ApJ, 347, 74

King, A., \& Pringle, J. 2007, MNRAS, 377, L25

Kollatschny, W., Biermann, P., Fricke, K. J., Huchtmeier, W., \& Witzel, A. 1983, A\&A, 119, 80

Krolik, J., \& Kriss, G. A. 1995, ApJ, 447, 512

Krolik, J. H. 1988, ApJ, 325, 148

Krolik, J. H., McKee, C. F., \& Tarter, C. B. 1981, ApJ, 249, 422

Kwan, J., \& Krolik, J. H. 1979, ApJ, 233, L91

Laor, A. 2006, ApJ, 643, 112

Laor, A. 2007, in ASP Conf. Ser. 373, Central Engine of Active Galactic Nuclei, ed. L. C. Ho \& J.-M. Wang (San Francisco, CA: ASP), 384

Laor, A., Barth, A. J., Ho, L. C., \& Filippenko, A. V. 2006, ApJ, 636, 83

Laor, A., \& Netzer, H. 1989, MNRAS, 238, 897

Levich, E. V., \& Sunyaev, R. A. 1970, ApJ, 7, 69

Magorrian, J., Tremaine, S., Richstone, D., et al. 1998, AJ, 115, 2285

Marziani, P., Sulentic, J. W., Negrete, C. A., et al. 2010, MNRAS, 409, 1033

Marziani, P., Sulentic, J. W., Stirpe, G. M., Zamfir, S., \& Calvani, M. 2009, A\&A, 495, 83

Marziani, P., Zamanov, R. K., Sulentic, J. W., \& Calvani, M. 2003, MNRAS, 345,1133

Mateos, S., Barcons, X., Carrera, F. J., et al. 2005, A\&A, 433, 855

Mathews, W. G. 1986, ApJ, 305, 187

Mathews, W. G., \& Blumenthal, G. R. 1977, ApJ, 214, 10

Mathews, W. G., \& Ferland, G. J. 1987, ApJ, 323, 456

Matsuoka, K., Nagao, T., Marconi, A., Maiolino, R., \& Taniguchi, Y. 2011, A\&A, 527, A100

McKee, C. F., \& Cowie, L. L. 1975, ApJ, 195, 715

Meerson, B. 1996, Rev. Mod. Phys., 68, 215

Meyer, F., \& Meyer-Hofmeister, E. 1994, A\&A, 288, 175

Murray, N., \& Chiang, J. 1995, ApJ, 454, L105

Murray, N., \& Chiang, J. 1997, ApJ, 474, 91

Netzer, H. 1980, ApJ, 236, 406

Netzer, H. 1985, ApJ, 289, 451

Netzer, H. 1990, in Active Galactic Nuclei, ed. R. Blandford, H. Netzer, \& L. Woljter (Berlin: Springer), 57

Netzer, H. 1996, ApJ, 473, 781

Netzer, H. 2008, New Astron. Rev., 52, 257

Netzer, H., \& Marziani, P. 2010, ApJ, 724, 318

Netzer, H., Shemmer, O., Maiolino, R., et al. 2004, ApJ, 614, 558

Netzer, H., Turner, T. J., \& George, I. M. 1998, ApJ, 504, 680
Nicastro, F., Martocchia, A., \& Matt, G. 2003, ApJ, 589, L13

Nipoti, C. 2010, MNRAS, 406, 247

Osterbrock, D., \& Ferland, G. (ed.) 2006, Astrophysics of Gaseous Nebulae and Active Galactic Nuclei (Sausalito, CA: Univ. Science Books), 344

Osterbrock, D., \& Mathews, W. 1986, ARA\&A, 24, 171

Panessa, F., \& Bassani, L. 2002, A\&A, 394, 435

Panessa, F., Carrera, F. J., Bianchi, S., et al. 2009, MNRAS, 398, 1951

Papaloizou, J. C. B., \& Pringle, J. E. 1984, MNRAS, 208, 721

Pappa, A., Georgantopoulos, I., Stewart, G. C., \& Zezas, A. L. 2001, MNRAS, 326, 995

Penston, M. V. 1988, MNRAS, 233, 601

Perry, J. J., \& Dyson, J. E. 1985, MNRAS, 213, 665

Peterson, B. 1997, An Introduction to Active Galactic Nuclei (Cambridge: Cambridge Univ. Press), 102

Peterson, B. M., \& Wandel, A. 1999, ApJ, 521, L95

Petre, R., Mushotzky, R. F., Krolik, J. H., \& Holt, S. S. 1984, ApJ, 280, 499

Pittard, J. M., Dyson, J. E., Falle, S., \& Hartquist, T. W. 2001, A\&A, 375, 827

Pittard, J. M., Hartquist, T. W., \& Ashmore, I. 2003, A\&A, 408, 813

Rafikov, R. 2009, ApJ, 704, 281

Rees, M., Netzer, H., \& Ferland, G. 1989, ApJ, 347, 647

Reynolds, C. 1997, MNRAS, 286, 513

Reynolds, C., \& Fabian, A. C. 1995, MNRAS, 273, 1167

Richards, G., Lacy, M., Storrie-Lombardi, L. J., et al. 2006, ApJS, 166, 470

Risaliti, G. 2010, IAU Symp. 267, Co-Evolution of Central Black Holes and Galaxies, ed. B. M. Peterson, R. S. Somerville, \& T. Storchi-Bergmann (Cambridge: Cambridge Univ. Press), 299

Roos, N. 1992, ApJ, 385, 108

Rybicki, G. B., \& Lightman, A. P. 1979, Radiative Processes in Astrophysics (New York: Wiley)

Scoville, N., \& Norman, C. 1988, ApJ, 332, 163

Shakura, N. I., \& Sunyaev, R. A. 1973, A\&A, 24, 337

Shemmer, O., Netzer, H., Maiolino, R., et al. 2004, ApJ, 614, 547

Shemmer, O., Trakhtenbrot, B., Anderson, S. F., et al. 2010, ApJ, 722, L152

Shields, G. A. 1977, Astrophys. Lett., 18, 119

Sirko, E., \& Goodman, J. 2003, MNRAS, 341, 501

Shlosman, I., Vitello, P. A., \& Shaviv, G. 1985, ApJ, 294, 96

Simon, L. E., \& Hamann, F. 2010, MNRAS, 407, 1826

Smith, M. D., \& Raine, D. J. 1985, MNRAS, 212, 425

Spitzer, L. 1962, Physics of Fully Ionized Gases (New York: Interscience), 87

Sulentic, J. W., Marziani, P., \& Dultzin-Hacyan, D. 2000, ARA\&A, 38, 521

Sutherland, R. S., \& Dopita, M. A. 1993, ApJS, 88, 253

Terlevich, R., Tenorio-Tagle, G., Franco, J., \& Melnick, J. 1992, MNRAS, 255, 713

Thompson, T., Quataert, E., \& Murray, N. 2005, ApJ, 630, 167

Torricelli-Ciamponi, G., \& Pietrini, P. 2002, A\&A, 349, 215

Tout, C. A., Eggleton, P. P., Fabian, A. C., \& Pringle, J. E. 1989, MNRAS, 238 , 427

Tran, H. 2001, ApJ, 554, 19

Tran, H., Lyke, J. E., \& Mader, J. A. 2011, ApJ, 726, 21

Tremaine, S., Gebhardt, K., Bender, R., et al. 2002, ApJ, 574, 740

Trippe, M. L., Crenshaw, D. M., Deo, R. P., et al. 2010, ApJ, 725, 1749

Turner, T. J., George, I. M., \& Mushotzky, R. F. 1993, ApJ, 412, 72

Turner, T. J., George, I. M., Nandra, K., \& Mushotzky, R. F. 1997, ApJS, 113, 23

Vanden Berk, D. E., Richards, G. T., Bauer, A., et al. 2001, AJ, 122, 549

Vasudevan, R. V., Mushotzky, R. F., Winter, L. M., \& Fabian, A. C. 2009, MNRAS, 399, 1553

Vignali, C., Alexander, D. M., \& Comastri, A. 2006, MNRAS, 373, 321

Vilkoviskij, E. Y., \& Czerny, B. 2002, A\&A, 387, 804

Wandel, A., Milgrom, M., \& Yahil, A. 1985, ApJ, 292, 206

Wang, J.-M., Ge, J.-Q., Hu, C., et al. 2011, ApJ, 739, 3

Wang, J.-M., Hu, C., Li, Y.-R., et al. 2009, ApJ, 697, L141

Wang, J.-M., Watarai, K., \& Mineshige, S. 2004, ApJ, 607, L107

Wang, J.-M., Yan, C.-S., Gao, H.-Q., et al. 2010, ApJ, 719, L148

Wang, J.-M., \& Zhang, E.-P. 2007, ApJ, 627, L5

Wang, J.-M., \& Zhou, Y.-Y. 1999, ApJ, 516, 420

Warner, C., Hamann, F., \& Dietrich, M. 2003, ApJ, 596, 72

Warner, C., Hamann, F., \& Dietrich, M. 2004, ApJ, 608, 136

Whittle, M., \& Saslaw, W. C. 1986, ApJ, 310, 104

Wolter, A., Gioia, I. M., Henry, J. P., \& Mullis, C. R. 2005, A\&A, 444, 165

Woltjer, L. 1959, ApJ, 130, 38

Woods, D. T., Klein, R. I., Castor, J. I., McKee, C. F., \& Bell, J. B. 1996, ApJ, 461,767

Zakamska, N. L., Schmidt, G. D., Smith, P. S., et al. 2005, AJ, 129, 1212

Zakamska, N. L., Strauss, M. A., Krolik, J. H., et al. 2003, AJ, 126, 2125

Zhang, E.-P., \& Wang, J.-M. 2006, ApJ, 653, 137

Zurek, W. H., Siemiginowska, A., \& Colgate, S. A. 1994, ApJ, 434, 46 